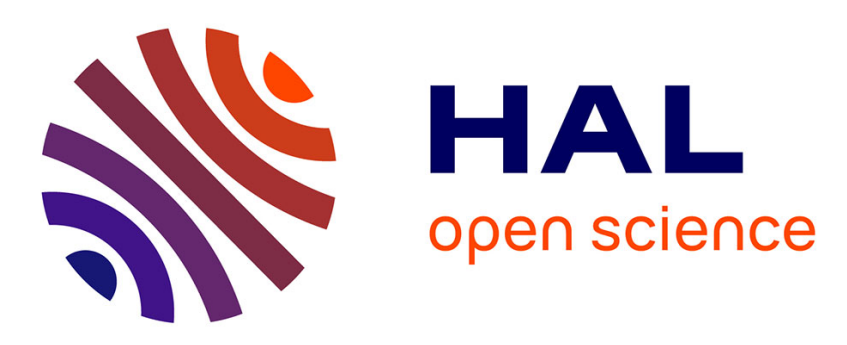

\title{
Properties of field functionals and characterization of local functionals
}

Christian Brouder, Nguyen Viet Dang, Camille Laurent-Gengoux, Kasia Rejzner

\section{To cite this version:}

Christian Brouder, Nguyen Viet Dang, Camille Laurent-Gengoux, Kasia Rejzner. Properties of field functionals and characterization of local functionals. Journal of Mathematical Physics, 2018, 59 (2), pp.023508. 10.1063/1.4998323 . hal-01654120v3

\section{HAL Id: hal-01654120 \\ https://hal.science/hal-01654120v3}

Submitted on 16 May 2019

HAL is a multi-disciplinary open access archive for the deposit and dissemination of scientific research documents, whether they are published or not. The documents may come from teaching and research institutions in France or abroad, or from public or private research centers.
L'archive ouverte pluridisciplinaire HAL, est destinée au dépôt et à la diffusion de documents scientifiques de niveau recherche, publiés ou non, émanant des établissements d'enseignement et de recherche français ou étrangers, des laboratoires publics ou privés. 


\section{Properties of field functionals and characterization of local functionals}

Cite as: J. Math. Phys. 59, 023508 (2018); https://doi.org/10.1063/1.4998323

Submitted: 31 July 2017 . Accepted: 25 January 2018 . Published Online: 20 February 2018

Christian Brouder, Nguyen Viet Dang, Camille Laurent-Gengoux, and Kasia Rejzner (D)

\section{ARTICLES YOU MAY BE INTERESTED IN}

Finite-part integration of the generalized Stieltjes transform and its dominant asymptotic behavior for small values of the parameter. I. Integer orders

Journal of Mathematical Physics 59, 023509 (2018); https://

doi.org/10.1063/1.5003479

New Fourier transform containing a pair of complex Euler $\Gamma$-functions with a monomial: Mathematical and physical applications

Journal of Mathematical Physics 59, 023510 (2018); https://

doi.org/10.1063/1.5024794

Half theory fractional angular momentum and the application of fractional derivatives to quantum mechanics

Journal of Mathematical Physics 59, 022110 (2018); https://

doi.org/10.1063/1.4990102

Where in the world is AIP Publishing?

Find out where we are exhibiting next 


\title{
Properties of field functionals and characterization of local functionals
}

\author{
Christian Brouder, ${ }^{1, a)}$ Nguyen Viet Dang, ${ }^{2}$ Camille Laurent-Gengoux, ${ }^{3}$ \\ and Kasia Rejzner ${ }^{4}$ \\ ${ }^{1}$ Sorbonne Université, Muséum National d'Histoire Naturelle, UMR CNRS 7509, \\ IRD, Institut de Minéralogie, de Physique des Matériaux et de Cosmochimie, IMPMC, \\ 75005 Paris, France \\ ${ }^{2}$ Institut Camille Jordan (UMR CNRS 5208), Université Claude Bernard Lyon 1, \\ Bât. Braconnier, 43 Blvd. du 11 Novembre 1918, 69622 Villeurbanne Cedex, France \\ ${ }^{3}$ Institut Elie Cartan de Lorraine, Université de Lorraine, Site de Metz, 3, \\ rue Augustin Fresnel, 57000 Metz - Technopole Metz, France \\ ${ }^{4}$ Department of Mathematics, University of York, Heslington, York YO10 5DD, United Kingdom
}

(Received 31 July 2017; accepted 25 January 2018; published online 20 February 2018)

\begin{abstract}
Functionals (i.e., functions of functions) are widely used in quantum field theory and solid-state physics. In this paper, functionals are given a rigorous mathematical framework and their main properties are described. The choice of the proper space of test functions (smooth functions) and of the relevant concept of differential (Bastiani differential) are discussed. The relation between the multiple derivatives of a functional and the corresponding distributions is described in detail. It is proved that, in a neighborhood of every test function, the support of a smooth functional is uniformly compactly supported and the order of the corresponding distribution is uniformly bounded. Relying on a recent work by Dabrowski, several spaces of functionals are furnished with a complete and nuclear topology. In view of physical applications, it is shown that most formal manipulations can be given a rigorous meaning. A new concept of local functionals is proposed and two characterizations of them are given: the first one uses the additivity (or Hammerstein) property, the second one is a variant of Peetre's theorem. Finally, the first step of a cohomological approach to quantum field theory is carried out by proving a global Poincaré lemma and defining multi-vector fields and graded functionals within our framework. Published by AIP Publishing. https://doi.org/10.1063/1.4998323
\end{abstract}

\section{MOTIVATION}

Functionals (i.e., functions of functions) are mathematical objects successfully applied in many areas of physics. Since Schwinger's ground-breaking papers, ${ }^{1,2}$ Green functions of quantum field theory are obtained as functional derivatives of the generating functional $Z(j)$ with respect to the functions $j$ (external sources). In solid-state and molecular physics, the exchange and correlation potential of density functional theory is computed from the functional derivative of the total energy $E(\rho)$ with respect to the electron density $\rho \cdot{ }^{3,4}$ In perturbative algebraic quantum field theory (pAQFT), the observables are functionals $F(\varphi)$ of the classical field $\varphi .^{5}$ This formulation was possible due to the crucial result ${ }^{6}$ that allowed us to realize abstract quantum fields as concrete functionals on the space of classical configurations. This viewpoint is not only simplifying computations but also allows us to construct new perturbative and exact models of QFT's. ${ }^{7,8}$ It is, therefore, crucial to understand the functional analytic properties of classical functionals to be able to use these in quantization and obtain even more models. The importance of this endeavour is justified by the fact that presently we do not know any exact interacting QFT models in 4 spacetime dimensions.

\footnotetext{
a)Electronic mail: christian.brouder@upmc.fr
} 
Functionals are also used in pure mathematics, for example, loop space cohomology ${ }^{9}$ and infinite dimensional integrable systems: the hierarchy of commuting Hamiltonians for the Korteweg-de Vries equation is, for instance, made of functionals. ${ }^{10}$

In all these fields, the concept of locality is fundamental: the Lagrangian of quantum field theory is local and the counterterms of the renormalization process have to be local, the approximations of $E(\rho)$ used in practice are local, and it is an open question whether the true density functional $E(\rho)$ is local or not. Therefore, it is crucial to determine precisely what is meant by a local functional. According to the standard definition, ${ }^{11-14}$ if $\varphi$ is a classical field (i.e., a smooth section of a vector bundle over $M$ and we momentarily consider $M=\mathbb{R}^{d}$ for notational convenience), then a functional $F(\varphi)$ is local if it is of the form

$$
F(\varphi)=\int_{\mathbb{R}^{d}} d x f\left(x, \varphi(x), \partial_{\mu} \varphi(x), \ldots, \partial_{\mu_{1} \ldots \mu_{k}} \varphi(x)\right),
$$

where $f$ is a smooth compactly supported function with a finite number of arguments.

However, this definition of local functionals is not very handy in practice because it is global and sometimes too restrictive. For example, general relativity has no local gauge-invariant observables in the sense of Eq. (1), whereas it has local gauge-invariant observables when the concept of locality is slightly generalized, as discussed in Ref. 7 (see also the parallel work ${ }^{15}$ ). Note that the concept of locality presented in the present paper gives a proper topological framework for local functionals as understood by Refs. 16-18.

The present paper puts forth the following formulation of the concept of locality:

Definition I.1. Let $M$ be a manifold. ${ }^{19}$ Let $U$ be an open subset of $C^{\infty}(M)$. A smooth functional $F: U \rightarrow \mathbb{K}$ (where $\mathbb{K}=\mathbb{R}$ or $\mathbb{C}$ ) is said to be local if, for every $\varphi \in U$, there is a neighborhood $V$ of $\varphi$, an integer $k$, an open subset $\mathcal{V} \subset J^{k} M$, and a smooth function $f \in C^{\infty}(\mathcal{V})$ such that $x \in M \mapsto f\left(j_{x}^{k} \psi\right)$ is supported in a compact subset $K \subset M$ and

$$
F(\varphi+\psi)=F(\varphi)+\int_{M} f\left(j_{x}^{k} \psi\right) d x,
$$

whenever $\varphi+\psi \in V$ and where $j_{x}^{k} \psi$ denotes the $k$-jet of $\psi$ at $x$.

In other words, we require $F$ to be local in the sense of Eq. (1), but only around each $\varphi \in$ $U$ because the integer $k$ and the function $f$ can depend on the neighborhood $V$. In short, our local functionals are local in the "traditional sense," but only locally in the configuration space (i.e., in a neighborhood of each $\varphi$ ). We do not need global locality to apply variational methods and derive Euler-Lagrange equations. We will show by exhibiting an example that this concept of locality is strictly more general than the traditional one. Our first main result is a simple characterization of local functionals in the sense of Definition 1.1:

Theorem I.2. Let $U$ be an open subset of $C^{\infty}(M)$. A smooth functional $F: U \rightarrow \mathbb{K}$ (where $\mathbb{K}=\mathbb{R}$ or $\mathbb{C})$ is local if and only if we have the following:

1. $F$ is additive [i.e., it satisfies $F\left(\varphi_{1}+\varphi_{2}+\varphi_{3}\right)=F\left(\varphi_{1}+\varphi_{2}\right)+F\left(\varphi_{2}+\varphi_{3}\right)-F\left(\varphi_{2}\right)$ whenever supp $\left.\varphi_{1} \cap \operatorname{supp} \varphi_{3}=\emptyset\right]$.

2. For every $\varphi \in U$, the differential $D F_{\varphi}$ of $F$ at $\varphi$ is a distribution with empty wave front set. Thus, it can be represented by a function $\nabla F_{\varphi} \in \mathcal{D}(M)$ [with $\mathcal{D}(M)$ the space of compactly supported smooth functions on $M$, i.e., "test functions"].

3. The map $U \rightarrow \mathcal{D}(M)$ defined by $\varphi \mapsto \nabla F_{\varphi}$ is smooth (in the sense of Bastiani).

Our characterization of locality is inspired by the microlocal functionals proposed by Brunetti, Fredenhagen, and Ribeiro. ${ }^{20}$ However, the proof of their Proposition 2.3.12 is not complete because the application of the Fubini theorem and the second use of the fundamental theorem of calculus are not justified. Our condition 3 solves that problem. On the other hand, we do not need their assumption that $F$ is compactly supported.

Let us stress that the notion of locality is quite subtle and depends strongly on the functional analytic setting. A functional characterization of a notion of local functionals on measurable 
functions might not be valid anymore when applied to smooth functions as is shown by the simple counterexample of Sec. V B. We also make a conjecture as to how to generalize our main result to multi-vector fields and graded functionals, which is required for a rigorous version of the BatalinVilkovisky approach to gauge field theory and quantum gravity. The second main result is a proof of the global Poincare lemma (in our context), which is crucial to set up the BRST (Becchi, Rouet, Stora, Tyutin) and variational complexes. The last one is another characterization of local (and multilocal) functionals in the form of a Peetre's theorem.

Along the way to these results, we prove interesting properties of general functionals that we briefly describe now. In Sec. II, we explain why we choose test functions that are only smooth instead of smooth and compactly supported, we describe the topology of the space of test functions, and we present the concept of Bastiani differentiability and its main properties. In Sec. III, we show that a smooth functional is locally compactly supported (i.e., in a neighborhood of every test function) and we prove that the $k$ th derivative of a functional defines a continuous family of distributions whose order is locally bounded. Section IV, which relies heavily on Dabrowski's work, ${ }^{21,22}$ describes in detail a nuclear and complete topology on several spaces of functionals used in quantum field theory. Section V discusses the concept of additivity that characterizes local functionals. Sections VI and VII prove the main results discussed above. Note that the present paper has a somewhat foundational character, in as much as the choice of test-functions, additivity property, and differential are carefully justified from the physical and mathematical points of view. It contributes to the formulation of a mathematically rigorous basis on which the quantum field theory of gauge fields and gravitation can be built.

Note also that this paper aims at both functional analysts and theoretical physicists. Because of this dual readership, the proofs are often more detailed than what would be required for experts in functional analysis.

\section{FUNCTIONALS AND THEIR DERIVATIVES}

To set up a mathematical definition of functionals, we need to determine precisely which space of test functions (i.e., classical fields and sources) we consider and what we mean by a functional derivative.

\section{A. The space of classical fields}

Propagators and Green functions of quantum fields in flat spacetimes are tempered distributions $^{23,24}$ and the corresponding test functions are rapidly decreasing. Tempered distributions are computationally convenient because they have Fourier transforms. However, tempered distributions cannot be canonically extended to curved spacetimes (i.e., smooth Lorentzian manifolds) because the rapid decrease of test functions at infinity is controlled by some Euclidian distance which is not canonically defined on general spacetime manifolds (Ref. 25, p. 339).

The most natural spaces of test functions on a general spacetime $M$ are the space $C^{\infty}(M)$ of real valued smooth functions on $M$ and its subspace $\mathcal{D}(M)$ of compactly supported functions. These two spaces are identical when $M$ is compact, but physically relevant spacetimes are not compact because they are globally hyperbolic, and a choice must be made.

In this paper, we choose $C^{\infty}(M)$ [or the set $\Gamma(M, B)$ of smooth sections of a vector bundle $B$ ]. There is a strong physical reason for this: ${ }^{26}$ in the quantization process, we must be able to deal with on-shell fields $\varphi$ that are smooth solutions to normally hyperbolic equations and as such cannot be compactly supported. Therefore, the domain of the functionals can be $C^{\infty}(M)$ but not $\mathcal{D}(M)$. There are also good mathematical arguments for this choice: Indeed, $C^{\infty}(M)$ is a Fréchet space and its pointwise multiplication is continuous (Ref. 27, p. 119). Moreover, the Fréchet property of $C^{\infty}(M)$ saves us the trouble of distinguishing Bastiani from convenient differentiability which is treated in Ref. 28.

The choice of $C^{\infty}(M)$ has, however, several drawbacks: (i) Since smooth functions are generally not integrable over $M$, the Lagrangian density $\mathcal{L}(\varphi)$ must be multiplied by a smooth compactly supported function $g$ so that $\mathcal{L}(\varphi) g$ is integrable over $M .{ }^{29}$ As a result, long-range interactions are 
suppressed and infrared convergence is enforced. This simplifies the problem but makes it difficult to deal with the physics of infrared divergence. (ii) The function $g$ breaks the diffeomorphism invariance of the Einstein-Hilbert action. (iii) The effect of a perturbation $\varphi+\epsilon \psi$ is easier to deal with when $\psi$ is compactly supported because it avoids the presence of boundary terms. This problem can be solved by considering $C^{\infty}(M)$ as a manifold modeled on $\mathcal{D}(M),{ }^{5,20}$ but this is an additional complication.

\section{B. Locally convex spaces}

The spaces of test functions and functionals considered in the paper are all locally convex. The most pedagogical introduction to locally convex spaces is probably Horvath's book, ${ }^{30}$ so we refer the reader to it for more details.

We describe now the topology of the spaces of test functions that we use. For the space of smooth test functions $C^{\infty}\left(\mathbb{R}^{d}\right)$, the topology is defined by the seminorms

$$
\pi_{m, K}(f)=\sup _{x \in K} \sup _{|\alpha| \leq m}\left|\partial^{\alpha} f(x)\right|,
$$

where $f \in C^{\infty}\left(\mathbb{R}^{d}\right), m$ is an integer, $K$ is a compact subset of $\mathbb{R}^{d}, \alpha=\left(\alpha_{1}, \ldots, \alpha_{d}\right)$ is a $d$-tuple of nonnegative integers, with $|\alpha|=\alpha_{1}+\cdots+\alpha_{d}$ and $\partial^{\alpha}=\partial_{1}^{\alpha_{1}} \ldots \partial_{d}^{\alpha_{d}}$, with $\partial_{i}=\partial / \partial x^{i}$ being the derivative with respect to the $i$ th coordinate of $x$ (Ref. 27, p. 88).

If $U$ is open in $\mathbb{R}^{d}$, we denote by $C^{\infty}(U)$ the space of all functions defined on $U$ which possess continuous partial derivatives of all orders. We equip $C^{\infty}(U)$ with the topology defined by the seminorms $\pi_{m, K}$ where $K$ runs now over the compact subsets of $U$ (Ref. 30, p. 89). For every open set $U \subset \mathbb{R}^{d}$, the space $C^{\infty}(U)$ is Fréchet, reflexive, Montel, barrelled (Ref. 30, p. 239), bornological (Ref. 30, p. 222), and nuclear (Ref. 31, p. 530).

We define now $C^{\infty}(M)$, where $M$ is a $d$-dimensional manifold (tacitly smooth, Hausdorff, paracompact, and orientable) described by charts $\left(U_{\alpha}, \psi_{\alpha}\right)$. If for every $U_{\alpha} \subset M$ we are given a smooth function $g_{\alpha} \in C^{\infty}\left(\psi_{\alpha}\left(U_{\alpha}\right)\right)$ such that $g_{\beta}=g_{\alpha} \circ \psi_{\alpha} \circ \psi_{\beta}^{-1}$ on $\psi_{\beta}\left(U_{\alpha} \cap U_{\beta}\right)$, we call the system $g_{\alpha}$ a smooth function $g$ on $M$. The space of smooth functions on $M$ is denoted by $C^{\infty}(M)(\operatorname{Ref}$. 32, p. 143). This definition is simple, but to describe the topological properties of $C^{\infty}(M)$, the following more conceptual definition is useful.

Let $M$ be a manifold and $B \rightarrow M$ be a smooth vector bundle of rank $r$ over $M$ with projection $\pi$. Let $E=\Gamma(M, B)$ be the space of smooth sections of $B$ equipped with the following topology: ${ }^{28}$

Definition II.1. The topology on $\Gamma(M, B)$ is defined as follows. Choose a chart $\left(U_{\alpha}, \psi_{\alpha}\right)_{\alpha}$ and a trivialization map $\Phi_{\alpha}: \pi^{-1}\left(U_{\alpha}\right) \rightarrow \Omega \times \mathbb{R}^{r}$, where $\Omega$ is a fixed open set in $\mathbb{R}^{d}$. Then the map $\Phi_{\alpha}$ allows us to identify $\Gamma\left(U_{\alpha}, B\right)$ with $C^{\infty}\left(\Omega, \mathbb{R}^{r}\right)$ by $\Phi_{\alpha}: \pi^{-1} U_{\alpha} \rightarrow \Omega \times \mathbb{R}^{d}$ such that

$$
\Phi_{\alpha}(x, s(x))=\left(\psi_{\alpha}(x), K_{\alpha}(s)\left(\psi_{\alpha}(x)\right)\right),
$$

where

$$
K_{\alpha}: s \in \Gamma\left(U_{\alpha}, B\right) \mapsto K_{\alpha}(s) \in C^{\infty}\left(\Omega, \mathbb{R}^{r}\right) .
$$

The topology on $\Gamma(M, B)$ is the weakest topology making all the maps $K_{\alpha}$ continuous.

This topology does not depend on the choice of charts or trivialization maps (Ref. 28, p. 294). To interpret this topology, denote by $\rho_{\alpha}:\left.s \in \Gamma(M, B) \mapsto s\right|_{U_{\alpha}} \in \Gamma\left(U_{\alpha}, B\right)$ the restriction map of sections on open sets of our open cover $\left(U_{\alpha}\right)_{\alpha \in I}$ of $M$. The space $\Gamma(M, B)$ fits into the following complex of vector spaces:

$$
0 \rightarrow \Gamma(M, B) \stackrel{\left(\rho_{\alpha}\right)_{\alpha \in I}}{\longrightarrow} \prod_{\alpha \in I} \Gamma\left(U_{\alpha}, B\right) \simeq \prod_{\alpha \in I} C^{\infty}\left(\Omega, \mathbb{R}^{r}\right) \stackrel{\left(\rho_{\alpha}-\rho_{\beta}\right)_{\alpha \neq \beta}}{\longrightarrow} \prod_{(\alpha \neq \beta) \in I^{2}} \Gamma\left(U_{\alpha} \cap U_{\beta}, B\right) .
$$

The topology on $\Gamma\left(U_{\alpha}, B\right)$ is given by the isomorphism $\Gamma\left(U_{\alpha}, B\right) \simeq C^{\infty}\left(\Omega, \mathbb{R}^{r}\right)$, hence it is nuclear Fréchet. The countable products $\prod_{\alpha \in I} \Gamma\left(U_{\alpha}, B\right)$ and $\prod_{(\alpha, \beta) \in I^{2}} \Gamma\left(U_{\alpha} \cap U_{\beta}, B\right)$ are therefore nuclear 
Fréchet. For every pair $(\alpha, \beta)$ of distinct elements of $I$, the difference of restriction maps $\rho_{\alpha}-\rho_{\beta}$ is continuous and the topology on $\Gamma(M, B)$ is the weakest topology which makes the above complex topological, which implies that it is nuclear Fréchet as the kernel of $\prod_{\alpha \neq \beta} \rho_{\alpha}-\rho_{\beta}$.

Locally convex spaces are very versatile and they are the proper framework to define spaces of smooth functionals, i.e., smooth functions on a space of functions (or sections of a bundle). The first step towards this goal is to provide a rigorous definition of functional derivatives.

\section{Functional derivatives}

To define the space of functionals, we consider the main examples $Z(j)$ and $F(\varphi)$. These two functionals send smooth classical fields to $\mathbb{K}$, where $\mathbb{K}=\mathbb{R}$ or $\mathbb{K}=\mathbb{C}$. Moreover, the functional derivatives of $Z$ and $F$ of all orders are required to obtain the Green functions from $Z(j)$ and to quantize the product $F(\varphi) G(\varphi)$. Therefore, we must define the derivative of a function $f: E \rightarrow \mathbb{K}$, where $E$ is the space of classical fields.

It will be useful to generalize the problem to functions $f$ between arbitrary locally convex spaces $E$ and $F$. To define such a derivative, we start from

Definition II.2. Let $U$ be an open subset of a Hausdorff locally convex space $E$ and let $f$ be a map from $U$ to a Hausdorff locally convex space $F$. Then $f$ is said to have a derivative at $x \in U$ in the direction of $v \in E$ if the following limit exists: ${ }^{33}$

$$
D f_{x}(v):=\lim _{t \rightarrow 0} \frac{f(x+t v)-f(x)}{t} .
$$

One can also consider the same definition restricted to $t>0 .{ }^{34}$ A function $f$ is said to have a Gâteaux differential ${ }^{35,36}$ (or a Gâteaux variation ${ }^{37}$ ) at $x$ if $D f_{x}(v)$ exists for every $v \in E$. However, this definition is far too weak for our purpose because $D f_{x}(v)$ is generally neither linear nor continuous in $v$ and it can be linear without being continuous and continuous without being linear (Ref. 38, p. 7). Therefore, we will use a stronger definition, namely, Bastiani differentiability, ${ }^{39}$ which is the fundamental concept of differentiability used throughout the paper:

Definition II.3. Let $U$ be an open subset of a Hausdorff locally convex space $E$ and let $f$ be a map from $U$ to a Hausdorff locally convex space $F$. Then $f$ is Bastiani differentiable on $U$ [denoted by $\left.f \in C^{1}(U)\right]$ iff has a Gâteaux differential at every $x \in U$ and the map $D f: U \times E \rightarrow F$ defined by $D f(x, v)=D f_{x}(v)$ is continuous on $U \times E$.

With this definition, most of the properties used in physics textbooks (e.g., chain rule, Leibniz rule, and linearity) are mathematically valid.

\section{Examples}

We shall consider several examples of functions from $C^{\infty}(M)$ to $\mathbb{R}$ or $\mathbb{C}$, where $M=\mathbb{R}^{d}$,

$$
\begin{aligned}
F(\varphi) & =\int_{M} f(x) \varphi^{n}(x) d x \\
G(\varphi) & =\int_{M^{n}} g\left(x_{1}, \ldots, x_{n}\right) \varphi\left(x_{1}\right) \ldots \varphi\left(x_{n}\right) d x_{1} \ldots d x_{n} \\
H(\varphi) & =\sum_{\mu \nu} \int_{M} g^{\mu \nu}(x) h(x) \partial_{\mu} \varphi(x) \partial_{\nu} \varphi(x) d x \\
I(\varphi) & =\int_{M} f(x) e^{\varphi(x)} d x \\
J(\varphi) & =e^{\int_{M} f(x) \varphi(x) d x} \\
K(\varphi) & =\int_{M} f(x) \sin (\varphi(x)) d x
\end{aligned}
$$


where $f, g$, and $h$ are smooth compactly supported functions and where $g$ is a symmetric function of its arguments. Further examples can be found in Refs. 17 and 40. It is immediate to check that

$$
\begin{aligned}
D F_{\varphi}(v) & =n \int_{M} g(x) \varphi^{n-1}(x) v(x) d x \\
D G_{\varphi}(v) & =n \int_{M^{n}} g\left(x_{1}, \ldots, x_{n}\right) \varphi\left(x_{1}\right) \ldots \varphi\left(x_{n-1}\right) v\left(x_{n}\right) d x_{1} \ldots d x_{n} \\
D H_{\varphi}(v) & =2 \sum_{\mu \nu} \int_{M} g^{\mu \nu}(x) h(x) \partial_{\mu} \varphi(x) \partial_{\nu} v(x) d x \\
D I_{\varphi}(v) & =\int_{M} f(x) e^{\varphi(x)} v(x) d x \\
D J_{\varphi}(v) & =e^{\int_{M} f(x) \varphi(x) d x} \int_{M} f(x) v(x) d x \\
D K_{\varphi}(v) & =\int_{M} f(x) \cos (\varphi(x)) v(x) d x
\end{aligned}
$$

\section{Historical remarks}

Definition II.3 is due to Bastiani ${ }^{39,41}$ and looks quite natural. In fact, it is not so. For a long time, many different approaches were tried. For any reasonable definition of differentiability, the map $D f_{x}$ : $E \rightarrow F$ is linear and continuous so that $D f_{x} \in L(E, F)$. If $E$ and $F$ are Banach spaces, then a map $f: E$ $\rightarrow F$ is defined to be continuously (Fréchet) differentiable if the map $x \rightarrow D f_{x}$ is continuous from $U$ to $L_{c}(E, F)$, where $L_{c}(E, F)$ is the set of continuous maps from $E$ to $F$ equipped with the operator norm topology. But Fréchet differentiability is strictly stronger than Bastiani's differentiability specialized to Banach spaces. ${ }^{42}$ This is why Bastiani's definition was often dismissed in the literature ${ }^{43}$ and, for locally convex spaces that are not Banach, the map $D f$ was generally required to be continuous from $U$ to $L(E, F)$ equipped with some well-chosen topology. However, when $E$ is not normable, no topology on $L(E, F)$ provides the nice properties of Bastiani's definition (Ref. 44, p. 6) [Hamilton (Ref. 40, p. 70 ) gives a simple example of a map which is continuous $U \times E \rightarrow E$ but such that the corresponding map $U \rightarrow L(E, E)$ is not continuous]. Thus, $L(E, F)$ was equipped with various non-topological convergence structures (Ref. 44, p. 23). The result is an impressive zoology of differentiabilities. Twenty-five of them were reviewed and classified by Averbukh and Smolyanov. ${ }^{45}$ Still more can be found in the extensive lists given by Gähler ${ }^{46}$ and Ver Eecke ${ }^{47}$ covering the period up to 1983 (see also Refs. 38, 44, and 48).

Nowadays, essentially two concepts of differentiability survive: Bastiani's and the so-called convenient approach developed by Kriegl-Michor in the reference monograph, ${ }^{28}$ which is weaker than Bastiani's for general Hausdorff locally convex spaces. In particular, on any locally convex space that is not bornological, there is a conveniently smooth map that is not continuous (Ref. 41, p. 19). However, a nice feature of both approaches is that for a Fréchet space $E$, a function $f: E \rightarrow \mathbb{K}$ is smooth in the sense of Bastiani if and only if it is smooth in the sense of the convenient calculus. ${ }^{49}$ Bastiani differentiability became widespread after it was used by Michor, ${ }^{50}$ Hamilton $^{40}$ (for Fréchet spaces), and Milnor ${ }^{51}$ and it is now vigorously developed by Glöckner and Neeb (see also Ref. 52).

To complete this section, we would like to mention that the Bastiani differential is sometimes called the Michal-Bastiani differential ${ }^{53-55}$ (or even Michel-Bastiani differential ${ }^{54}$ ). This is not correct. The confusion comes from the fact that Bastiani defines her differentiability in several steps. She starts from the Gâteaux derivability and then she says that a map $f: U \rightarrow F$ is differentiable at $x$ (see Ref. 41, p. 18 and Ref. 39, p. 18) if (i) $D f_{x}$ is linear and continuous from $E$ to $F$ and (ii) the map $m_{x}: \mathbb{R} \times E \rightarrow F$ defined by

$$
m_{x}(t, v)=\frac{f(x+t v)-f(x)}{t}-D f_{x}(v)
$$


for $x+t v \in U$, is continuous at $(0, v)$ for all $v \in E$. This differentiability at $x$ is indeed equivalent to the differentiability defined by Michal ${ }^{56}$ in 1938, as proved in Refs. 45 and 57, Ref. 44, p. 72, and Ref. 47, p. 202. What we call Bastiani differentiability is called differentiability on an open set by Bastiani (see Ref. 41, p. 25 and Ref. 39, p. 44) and is strictly stronger than Michal differentiability.

The same distinction between Michal-Bastiani differentiability and Bastiani differentiability is made by Keller (Ref. 44, p. 72) in his thorough review. Bastiani's differentiability is denoted by $C_{c}^{1}$ by Keller, ${ }^{44}$ who also attributes the definition equivalent to $C_{c}^{1}$ to Bastiani alone (Ref. 44, p. 11).

In her Ph.D. thesis, Andrée Bastiani developed her concept of differentiability to define distributions on a locally convex space $E$ with values in a locally convex space $F$. She started from Schwartz' remark that a distribution is, locally, the derivative of a continuous function. She used her differential $D$ to define $F$-valued distributions over $E .^{58}$ A drawback of Bastiani's framework with respect to the convenient framework is that her category is not Cartesian closed for locally convex spaces that are not Fréchet.

\section{Properties of the differential}

We review now some of the basic properties of functional derivatives which will be used in the sequel. We strongly recommend Hamilton's paper, ${ }^{40}$ adapted to locally convex spaces by Neeb. ${ }^{42}$

\section{Continuity}

We characterize continuous (nonlinear) maps between two locally convex spaces.

Lemma II.4. Let $E$ and $F$ be locally convex spaces whose topology is defined by the families of seminorms $\left(p_{i}\right)_{i \in I}$ and $\left(q_{j}\right)_{j \in J}$, respectively. Then $f$ is continuous at $x$ if and only if, for every seminorm $q_{j}$ of $F$ and every $\epsilon>0$, there is a finite number $\left\{p_{i_{1}}, \ldots, p_{i_{k}}\right\}$ of seminorms of $E$ and $k$ strictly positive numbers $\eta_{1}, \ldots, \eta_{k}$ such that $p_{i_{1}}(x-y)<\eta_{1}, \ldots, p_{i_{k}}(x-y)<\eta_{k}$ imply $q_{j}(f(y)-f(x))<\epsilon$.

Proof. This is just the translation in terms of seminorms of the fact that $f$ is continuous at $x$ if, for every open set $V$ containing $f(x)$, there is an open set $U$ containing $x$ such that $f(U) \subset V$ (Ref. 59, p. 86).

When the seminorms of $E$ are saturated (Ref. 30, p. 96), as the seminorms $\pi_{m, K}$ of $C^{\infty}\left(\mathbb{R}^{d}\right)$, the condition becomes simpler: a map $f: C^{\infty}\left(\mathbb{R}^{d}\right) \rightarrow \mathbb{K}$ is continuous at $x$ if and only if, for every $\epsilon>0$, there is a seminorm $\pi_{m, K}$ and an $\eta>0$ such that $\pi_{m, K}(x-y)<\eta$ implies $|f(y)-f(x)|<\epsilon$. Since Fréchet spaces are metrizable, we can also use the following characterization of continuity (Ref. 60, p. 154):

Proposition II.5. Let E be a metrizable topological space and $F$ be a topological space. Then, a map $f: E \rightarrow F$ is continuous at a point $x$ if and only if, whenever a sequence $\left(x_{n}\right)_{n \in \mathbb{N}}$ converges to $x$ in $E$, the sequence $f\left(x_{n}\right)_{n \in \mathbb{N}}$ converges to $f(x)$ in $F$.

Another useful theorem is (Ref. 61, p. III.30)

Proposition II.6. Let $E$ and $F$ be two Fréchet spaces and $G$ be a locally convex space. Every separately continuous bilinear mapping from $E \times F$ to $G$ is continuous.

This result extends to multilinear mappings from a product $E_{1} \times \cdots \times E_{n}$ of Fréchet spaces to a locally convex space. ${ }^{62,63}$

\section{The fundamental theorem of calculus}

The fundamental theorem of calculus for functionals reads 
Theorem II.7. Let $f$ be a Bastiani differentiable map between two Hausdorff locally convex spaces $E$ and $F$. Let $U$ be an open set in $E, x$ in $U$, and $v$ in $E$ such that $(x+t v) \in U$ for every $t$ in an open neighborhood I of $[0,1]$ so that $g: t \mapsto f(x+t v)$ is a map from I to $F$. Then,

$$
f(x+v)=f(x)+\int_{0}^{1} g^{\prime}(t) d t=f(x)+\int_{0}^{1} D f_{x+t v}(v) d t .
$$

To give a meaning to Eq. (4), we need to define an integral of a function taking its values in a locally convex space. To cut a long story short: ${ }^{64,65}$

Definition II.8. Let X be a locally compact space (for example, $\mathbb{R}^{d}$ or some finite dimensional manifold), $\mu$ be a measure on $X$, and $F$ be a Hausdorff locally convex space. Let $f$ be a compactly supported continuous function from $X$ to $F$. Let $F^{\prime}$ be the topological dual of $F$ (i.e., the space of continuous linear maps from $F$ to $\mathbb{K})$. If there is an element $y \in F$ such that

$$
\langle\alpha, y\rangle=\int_{X}\langle\alpha, f\rangle d \mu
$$

for every $\alpha \in F^{\prime}$, where $\langle\cdot, \cdot\rangle$ denotes the duality pairing, then we say that $f$ has a weak integral and we denote y by $\int_{X} f d \mu$.

The uniqueness of the weak integral follows from the fact that $F$ is Hausdorff. In general, the existence of a weak integral requires some completeness property for $F$ (Ref. 64, p. 79). However, this is not the case for the fundamental theorem of calculus (Ref. 41, p. 27). This point was stressed by Glöckner. ${ }^{66}$

\section{Additional properties}

For maps between locally convex spaces, the linearity of the differential is not completely trivial. ${ }^{40}$

Proposition II.9. Let $E$ and $F$ be locally convex spaces and $f$ be a Bastiani differentiable map from an open subset $U$ of $E$ to $F$. Then, for every $x \in U$, the differential $D f_{x}: E \rightarrow F$ is a linear map.

The chain rule for Bastiani-differentiable functions was first proved by Bastiani herself ${ }^{41,39}$ (see also Ref. 53).

Proposition II.10. Assume that $E, F, G$ are locally convex spaces, $U \subset E$ and $V \subset F$ are open subsets, and $f: V \rightarrow G$ and $g: U \rightarrow V$ are two Bastiani-differentiable maps. Then, the composite map $f \circ g: U \rightarrow G$ is Bastiani differentiable and $D(f \circ g)_{x}=D f_{g(x)} \circ D g_{x}$.

By using these properties, the reader can prove that our examples are all Bastiani differentiable.

\section{E. Smooth functionals}

To define smooth functionals, we first define multiple derivatives.

Definition II.11. Let $U$ be an open subset of a locally convex space E and f be a map from $U$ to a locally convex space $F$. We say that fis $k$-times Bastiani differentiable on $U$ if we have the following:

- The k-th Gâteaux differential

$$
D^{k} f_{x}\left(v_{1}, \ldots, v_{k}\right)=\left.\frac{\partial^{k} f\left(x+t_{1} v_{1}+\cdots+t_{k} v_{k}\right)}{\partial t_{1} \ldots \partial t_{k}}\right|_{\mathbf{t}=0},
$$

where $\mathbf{t}=\left(t_{1}, \ldots, t_{k}\right)$, exists for every $x \in U$ and every $v_{1}, \ldots, v_{k} \in E$.

- The map $D^{k} f: U \times E^{k} \rightarrow F$ is continuous. 
Notice that for a function $f$ assumed to be $k$-times Bastiani differentiable, the restriction to any finite dimensional affine subspace is not only $k$-times differentiable (in the usual sense) but indeed of class $C^{k}$. The set of $k$-times Bastiani differentiable functions on $U$ is denoted by $C^{k}(U)$ or $C^{k}(U, F)$ when the target space $F$ has to be specified. Bastiani gives an equivalent definition, called $k$-times differentiability on $U$ (Ref. 41, p. 40), which is denoted by $C_{c}^{k}$ by Keller. ${ }^{44}$

Definition II.12. Let $U$ be an open subset of a locally convex space $E$ and $f$ be a map from $U$ to a locally convex space $F$. We say that $f$ is smooth on $U$ if $f \in C^{k}(U, F)$ for every integer $k$.

We now list a number of useful properties of the $k$ th Bastiani differential:

Proposition II.13. Let $U$ be an open subset of a locally convex space $E$ and $f \in C^{k}(U, F)$, where $F$ is a locally convex space, then we have the following:

1. $D^{k} f_{x}\left(v_{1}, \ldots, v_{k}\right)$ is a k-linear symmetric function of $v_{1}, \ldots, v_{k}($ Ref. 40, p. 84).

2. The function $f$ is of class $C^{m}$ for all $0 \leq m \leq k$ (Ref. 41, p. 40). In particular, $f$ is continuous.

3. The compositions of two functions in $C^{k}$ is in $C^{k}$ and the chain rule holds (Ref. 41, p. 51 and Ref. 40, p. 84).

4. The map $D^{m} f$ is in $C^{k-m}\left(U, L\left(E^{m}, F\right)\right)($ Ref. $41, p .40)$ where $L\left(E^{m}, F\right)$ is the space of jointly continuous m-linear maps from $E$ to $F$, equipped with the locally convex topology of uniform convergence on the compact sets of E, i.e., the topology generated by the seminorms

$$
p_{C, j}(\alpha)=\sup _{\left(h_{1}, \ldots, h_{m}\right) \in C} q_{j}\left(\alpha\left(h_{1}, \ldots, h_{m}\right)\right)
$$

where $C=C_{1} \times \cdots \times C_{m}, C_{i}$ runs over the compact sets of $E$ and $\left(q_{j}\right)_{j \in J}$ is a family of seminorms defining the topology of $F$.

5. If $E$ is metrizable, then $f \in C^{k}(U, F)$ if and only if $f$ belongs to $C^{k-1}(U, F)$ and $D^{k-1} f: U \rightarrow$ $L\left(E^{k-1}, F\right)$ is Bastiani differentiable (Ref. 41, p. 43). Here the metrizability hypothesis is used to obtain a canonical injection from $C\left(U \times E, L\left(E^{k-1}, F\right)\right)$ to $C\left(U \times E^{k}, F\right)$.

We refer the reader to Refs. 40 and 42 and Bastiani's cited studies for the proofs. Other results on $C^{k}(U)$ functions can be found in Keller's book. ${ }^{44}$ All the statements of Proposition II.13 are valid for $k=\infty$, i.e., smooth functions. Bastiani also defines jets of smooth functions between locally convex spaces (Ref. 41, p. 52 and Ref. 39, p. 75).

Note that $\mathrm{Neeb}^{67}$ and Glöckner ${ }^{68}$ agree with Bastiani for the definition of the first derivative but they use an apparently simpler definition of higher derivatives by saying that $f$ is $C^{k}$ if and only if $f$ is $C^{k-1}$ and $d^{k-1} f$ is $C^{1}$. However, this definition is less natural because, for example, $f \in C^{2}$ if $d f$ : $U \times E \rightarrow F$ is $C^{1}$. In the definition of the first derivative, $U$ is now replaced by $U \times E$ and $E$ by $E \times$ $E$. In other words, $d^{2}$ is a continuous map from $U \times E^{3}$ to $F$. More generally $d^{k}$ is a continuous map from $U \times E^{2^{k}-1}$ to $F$ (Ref. 68, p. 20). Moreover, according to Proposition 1.3.13 (Ref. 68, p. 23), a map $f$ belongs to $C^{k}$ if and only if it belongs to $C^{k}(U)$ is the sense of Bastiani, and Bastiani's $D^{k} f$ is denoted by $d^{(k)} f$ by Glöckner (Ref. 68, p. 23) and called the $k$ th differential of $f$. The $k$ th derivatives $d^{k} f$ and $d^{(k)} f=D^{k} f$ are not trivially related. For example (Ref. 68, p. 24), $d^{2} f\left(x, h_{1}, h_{2}, h_{3}\right)=D^{2} f(x$, $\left.h_{1}, h_{2}\right)+D f\left(x, h_{3}\right)$.

The Taylor formula with remainder for a function in $C^{n+1}(U)$ reads (Ref. 41, p. 44)

$$
\begin{aligned}
f(x+t h) & =f(x)+\sum_{k=1}^{n} \frac{t^{k}}{k !} D^{k} f_{x}\left(h^{k}\right)+\int_{0}^{t} \frac{(t-\tau)^{n}}{n !} D^{n+1} f_{x+\tau h}\left(h^{n+1}\right) d \tau \\
& =f(x)+\sum_{k=1}^{n} \frac{t^{k}}{k !} D^{k} f_{x}\left(h^{k}\right)+\int_{0}^{t} \frac{(t-\tau)^{n-1}}{(n-1) !}\left(D^{n} f_{x+\tau h}\left(h^{n}\right)-D^{n} f_{x}\left(h^{n}\right)\right) d \tau .
\end{aligned}
$$


Taylor's formula with remainder is a very important tool to deal with smooth functions on locally convex spaces.

The reader can check that all our examples are smooth functionals in the sense of Bastiani,

$$
D^{k} F_{\varphi}\left(v_{1}, \ldots, v_{k}\right)=\frac{n !}{(n-k) !} \int_{M} f(x) \varphi^{n-k}(x) v_{1}(x) \ldots v_{k}(x) d x,
$$

for $k \leq n$ and $D^{k} F_{\varphi}=0$ for $k>n$, and

$$
D^{k} G_{\varphi}\left(v_{1}, \ldots, v_{k}\right)=\frac{n !}{(n-k) !} \int_{M^{n}} g\left(x_{1}, \ldots, x_{n}\right) v_{1}\left(x_{1}\right) \ldots v_{k}\left(x_{k}\right) \varphi\left(x_{k+1}\right) \ldots \varphi\left(x_{n}\right) d x_{1} \ldots d x_{n},
$$

for $k \leq n$ and $D^{k} G_{\varphi}=0$ for $k>n$. Recall that $g$ is a symmetric, smooth compactly supported function of its arguments. The functional $H$ has only two non-zero derivatives and

$$
D^{2} H_{\varphi}\left(v_{1}, v_{2}\right)=2 \sum_{\mu \nu} \int_{M} g^{\mu \nu}(x) h(x) \partial_{\mu} v_{1}(x) \partial_{\nu} v_{2}(x) d x .
$$

The example $I$ has an infinite number of nonzero derivatives,

$$
D^{k} I_{\varphi}\left(v_{1}, \ldots, v_{k}\right)=\int_{M} f(x) e^{\varphi(x)} v_{1}(x) \ldots v_{k}(x) d x
$$

Finally

$$
D^{k} J_{\varphi}\left(v_{1}, \ldots, v_{k}\right)=e^{\int_{M} f(x) \varphi(x) d x} \int_{M^{k}} f\left(x_{1}\right) \ldots f\left(x_{k}\right) v_{1}\left(x_{1}\right) \ldots v_{k}\left(x_{k}\right) d x_{1} \ldots d x_{k}
$$

The functionals $F, G$, and $H$ are polynomials in the sense of Bastiani (Ref. 41, p. 53):

Definition II.14. Let E and $F$ be locally convex spaces. A polynomial of degree $n$ on $E$ is a smooth function $f: E \rightarrow F$ such that $D^{k} f=0$ for all $k>n$.

Let $u$ be a distribution in $\mathcal{D}^{\prime}\left(M^{k}\right)$, then the functional $f: \mathcal{D}(M) \rightarrow \mathbb{K}$ defined by $f(\varphi)=u\left(\varphi^{\otimes k}\right)$ is polynomial in the sense of Bastiani and its $k$-derivative is

$$
D^{k} f_{\varphi}\left(v_{1}, \ldots, v_{k}\right)=\sum_{\sigma} u\left(v_{\sigma(1)} \otimes \cdots \otimes v_{\sigma(k)}\right)
$$

where $\sigma$ runs over the permutations of $\{1, \ldots, k\}$ and the canonical inclusion $\mathcal{D}(M)^{\otimes k} \subset \mathcal{D}\left(M^{k}\right)$ was used.

If $F$ and $G$ are smooth maps from $E$ to $\mathbb{K}$, we can compose the smooth map $\varphi \mapsto(F(\varphi), G(\varphi))$ and the multiplication in $\mathbb{K}$ to show that

Proposition II.15. Let $E$ be a locally convex space and $U$ be an open set in E. Then the space of smooth functionals from $U$ to $\mathbb{K}$ is a sub-algebra of the algebra of real valued functions.

\section{PROPERTIES OF FUNCTIONALS}

We now prove important properties of smooth functionals. We first investigate the support of a functional. The fact that $D F_{\varphi}$ is continuous from $C^{\infty}(M)$ to $\mathbb{K}$ exactly means that $D F_{\varphi}$ is a compactly supported distribution for every $\varphi$. The support of $F$ is then essentially the union over $\varphi$ of the supports of $D F_{\varphi}$. We prove that, for any smooth functional and any $\varphi \in C^{\infty}(M)$, there is a neighborhood $V$ of $\varphi$ such that $\left.F\right|_{V}$ is compactly supported. 
The second property that we investigate is required to establish a link with quantum field theory. In this paper, we deal with functionals that are smooth functions $F$ on an open subset $U$ of $E=\Gamma(M$, $B)$, where $\Gamma(M, B)$ is the space of smooth sections of some finite rank vector bundle $B$ on the manifold $M$. There is a discrepancy between $D^{k} F_{\varphi}$, which is a continuous multilinear map from $E^{k}$ to $\mathbb{K}$, and the quantum field amplitudes (e.g., represented pictorially by Feynman diagrams) that are continuous linear maps from $E^{\hat{\otimes}_{\pi} k}=\Gamma\left(M^{k}, B^{\otimes k}\right)$ to $\mathbb{K}$, i.e., elements of the space $\Gamma^{\prime}\left(M^{k},\left(B^{*}\right)^{\otimes k}\right)$ of compactly supported distributions with values in the $k$ th external tensor power of the dual bundle $B^{*}$. It is easy to see that there is a canonical correspondence between $D^{k} F_{\varphi}$ and its associated distribution on $E^{\hat{\otimes}_{\pi} k}$, which we denote by $F_{\varphi}^{(k)}$. However, the equivalence between the continuity of $D^{k} F$ on $U \times E^{k}$ and the continuity of $F^{(k)}$ on $U \times E^{\hat{\otimes}_{\pi} k}$ requires a proof.

Finally, we show that the order of $F^{(k)}$ is locally bounded.

\section{A. Support of a functional}

Brunetti, Dütsch, and Fredenhagen proposed to define the support of a functional $F$ by the property that if the support of the smooth function $\psi$ does not meet the support of $F$, then $F(\varphi+\psi)$ $=F(\varphi)$ for all $\varphi$. More precisely, ${ }^{17}$

Definition III.1. Let $F: U \rightarrow \mathbb{K}$ be a Bastiani smooth function, with $U$ being a subset of $C^{\infty}(M)$. The support of $F$ is the set of points $x \in M$ such that, for every open set $\mathcal{U}_{x}$ containing $x$, there is a $\varphi$ $\in U$ and $a \psi$ in $C^{\infty}(M)$ with $\varphi+\psi \in U$ such that $\operatorname{supp} \psi \subset \mathcal{U}_{x}$ and $F(\varphi+\psi) \neq F(\varphi)$.

We want to relate this definition of the support of $F$ with the support of $D_{\varphi} F$, which is compactly supported as every distribution over $C^{\infty}(M) .{ }^{32}$ To do so, we need a technical lemma about connected open subsets in locally convex spaces.

Lemma III.2. Let $U$ be a connected open set in a locally convex space $E$, then any pair $(x, y) \in$ $U^{2}$ can be connected by a piecewise affine path.

Proof. Define the equivalence relation $\sim$ in $E$ as follows: two elements $(x, y)$ are equivalent if and only if they are connected by a piecewise affine path. Let us prove that this equivalence relation is both open and closed, hence any non-empty equivalence class for $\sim$ is both open and closed in $U$ and hence is equal to $U$.

Let $x \in U$, then there exists a convex neighborhood $V$ of $x$ in $U$ which means that every element in $V$ lies in the class of $x$, and the relation is open. Conversely let $y$ be in the closure of the equivalence class of $x$, then any neighborhood $V$ of $y$ contains an element equivalent to $x$. Choose some convex neighborhood $V$, then we find $z \in V$ such that $z \sim x$, but $z \sim y$ hence $x \sim z \sim y$ and we just proved that the equivalence class of $x$ was closed.

We can now prove an alternative formula due to Brunetti, Fredenhagen, and Ribeiro. ${ }^{20}$

Lemma III.3. For every Bastiani smooth function $F: U \rightarrow \mathbb{K}$, with $U$ being a connected open subset of $C^{\infty}(M)$,

$$
\operatorname{supp}(F)=\overline{\bigcup_{\varphi \in U} \operatorname{supp} D F_{\varphi}}
$$

Proof. Using the result of Lemma III.2, we may reduce to the case where $U$ is an open convex set. We prove that both sets $\overline{\bigcup_{\varphi} \operatorname{supp} D F_{\varphi}}$ and supp $F$ as defined in Definition III.1 have identical complements. Indeed, for every point $x \in M, x \notin \operatorname{supp} F$ means by definition of the support that there exists an open neighborhood $\Omega$ of $x$ such that $\forall(\psi, \varphi) \in \mathcal{D}(\Omega) \times C^{\infty}(M), F(\varphi+\psi)=F(\varphi)$. It follows that for all $\psi \in \mathcal{D}(\Omega)$, there exists $\varepsilon>0$ such that $|t| \leqslant \varepsilon \Longrightarrow \varphi+t \psi \in U$ and $t \in[-\varepsilon, \varepsilon] \mapsto F(\varphi+t \psi)$ is a constant function of $t$; therefore, $\left.\frac{d F(\varphi+t \psi)}{d t}\right|_{t=0}=D F_{\varphi}(\psi)=0$. This means that for all $\varphi \in U$, the support of $D F_{\varphi} \in \mathcal{E}^{\prime}(M)$ does not meet $\Omega$ since $\Omega$ lies in the complement of $\cup_{\varphi \in U} \operatorname{supp}\left(D F_{\varphi}\right)$ and therefore $x \in \Omega$ does not meet the closure $\overline{\cup_{\varphi \in U} \operatorname{supp}\left(D F_{\varphi}\right)}$. 
Conversely if $x$ does not meet the closure $\overline{\cup_{\varphi \in U} \operatorname{supp}\left(D F_{\varphi}\right)}$, then there is some neighborhood $\Omega$ of $x$ which does not meet $\cup_{\varphi \in U} \operatorname{supp}\left(D F_{\varphi}\right)$; therefore, for all $(\varphi, \psi) \in U \times \mathcal{D}(\Omega)$ such that $\varphi+\psi \in$ $U$, the whole straight path $[\varphi, \varphi+\psi]$ lies in $U$ (by convexity of $U$ ) and hence

$$
\forall t \in[0,1], D F_{\varphi+t \psi}(\psi)=0 \Longrightarrow \int_{0}^{1} d t D F_{\varphi+t \psi}(\psi)=0,
$$

and by the fundamental theorem of calculus, $F(\varphi+\psi)=F(\varphi)$.

Now we show that any smooth functional is locally compactly supported.

Proposition III.4. Let $F: U \mapsto \mathbb{K}$ be a Bastiani smooth function, where $U$ is an open connected subset of $E=C^{\infty}(M)$. For every $\varphi \in U$, there is a neighborhood $V$ of $\varphi$ in $U$ and a compact subset $K$ $\subset M$ such that the support of $F$ restricted to $V$ is contained in $K$. Moreover for all integers $n$ and all $\varphi \in U$, the distributional support $\operatorname{supp}\left(D^{n} F_{\varphi}\right)$ is contained in $K^{n} \subset M^{n}$.

Proof. By definition of the support of a functional, it is enough to show that, for every $\varphi \in V$, supp $D F_{\varphi} \subset K$. If $F$ is smooth, then $D F: U \times E \rightarrow \mathbb{K}$ is continuous. Thus, it is continuous in the neighborhood of $\left(\varphi_{0}, 0\right)$ for every $\varphi_{0} \in U$. In other words, for every $\epsilon>0$, there is a neighborhood $V$ of $\varphi_{0}$, a seminorm $\pi_{m, K}$, and an $\eta>0$ such that $\left|D F_{\varphi}(\chi)\right|<\epsilon$ for every $\varphi \in V$ and every $\chi \in E$ such that $\pi_{m, K}(\chi)<\eta$. Now, for every $\psi \in E$ such that $\pi_{m, K}(\psi) \neq 0$, we see that $\chi=\psi \eta /\left(2 \pi_{m, K}(\psi)\right)$ satisfies $\pi_{m, K}(\chi)<\eta$. Thus, $\left|D F_{\varphi}(\chi)\right|<\epsilon$ for every $\varphi \in V$ and, by linearity, $\left|D F_{\varphi}(\psi)\right|<(2 \epsilon / \eta) \pi_{m, K}(\psi)$. On the other hand, if $\pi_{m, K}(\psi)=0$, then for any $\mu>0 \psi_{m, K}(\mu \psi)=0<\eta$ so that $\left|D F_{\varphi}(\mu \psi)\right|<\epsilon$. By linearity, $\left|D F_{\varphi}(\psi)\right|<\epsilon / \mu$ for any $\mu>0$ and we conclude that $\left|D F_{\varphi}(\psi)\right|=0$. Thus, for every $\varphi \in V$ and every $\psi \in E$,

$$
\left|D F_{\varphi}(\psi)\right| \leq 2 \frac{\epsilon}{\eta} \pi_{m, K}(\psi) .
$$

Of course, this inequality implies that $D F_{\varphi}(\psi)=0$ when $\psi$ is identically zero on the compact subset $K$.

Let us show that this implies that the support of $D F_{\varphi}$ is contained in $K$. To avoid possible problems at the boundary, take any compact neighborhood $K^{\prime}$ of $K$. Now, take an open set $\Omega$ in $M$ such that $\Omega \cap K^{\prime}=\emptyset$. Then, for every smooth function $\psi$ supported in $\Omega$, we have $\pi_{m, K}(\psi)=0$ because the seminorm $\pi_{m, K}$ takes only into account the points of $K$. As a consequence, the restriction of $D F_{\varphi}$ to $\Omega$ is zero, which means that $\Omega$ is outside the support of $D F_{\varphi}$ for every $\varphi \in V$. Thus, supp $\left.F\right|_{V} \subset$ $K$ because, for every $\varphi \in V$, the support of $D F_{\varphi}$ is included in every compact neighborhood of $K$. Finally we show that if $F$ is compactly supported, then all $D^{n} F_{\varphi}$ are compactly supported with supp $D^{n} F_{\varphi} \subset(\operatorname{supp} F)^{\times n}$. This is easily seen by the following cutoff function argument: if $F$ is compactly supported, then for every cutoff function $\chi$ equal to 1 on an arbitrary compact neighborhood of supp $F$, we have $F(\varphi)=F(\chi \varphi), \forall \varphi \in E$. Then it is immediate by definition of $D^{n} F_{\varphi}$ that

$$
\begin{aligned}
D^{n} F_{\varphi}\left(\psi_{1}, \ldots, \psi_{n}\right) & =\left.\frac{d^{n} F\left(\chi\left(\varphi+t_{1} \psi_{1}+\cdots+t_{n} \psi_{n}\right)\right)}{d t_{1} \ldots d t_{n}}\right|_{\mathbf{t}=0} \\
& =D^{n} F_{\chi \varphi}\left(\chi \psi_{1}, \ldots, \chi \psi_{n}\right),
\end{aligned}
$$

thus supp $D^{n} F_{\varphi} \subset \operatorname{supp} \chi^{\times n}$ for all test function $\chi$ such that $\left.\chi\right|_{\operatorname{supp} F}=1$ and therefore supp $D^{n} F_{\varphi} \subset$ $(\operatorname{supp} F)^{\times n}$ since $\bigcap_{\chi \mid \operatorname{supp} F=1} \operatorname{supp} \chi=\operatorname{supp} F$.

\section{B. A multilinear kernel theorem with parameters}

We work with $M$ a smooth manifold and $B \rightarrow M$ a smooth vector bundle of finite rank over $M$. Let $E=\Gamma(M, B)$ be its space of smooth sections and $U$ be an open subset of $E$. We consider smooth maps $F: E \mapsto \mathbb{K}$ where $\mathbb{K}$ is the field $\mathbb{R}$ or $\mathbb{C}$. In this section, we relate the Bastiani derivatives $D^{k} F$, which are $k$-linear on $\Gamma(M, B)$ to the distributions used in quantum field theory, which are linear on 
$\Gamma\left(M^{k}, B^{\varpi k}\right)$. Since the $k$ th derivative $D^{k} F$ of a smooth map is multilinear and continuous in the last $k$ variables, we can use the following result (Ref. 69, p. 471 and Ref. 70, p. 259):

Lemma III.5. Let $E$ be a Hausdorff locally convex space. There is a canonical isomorphism between any $k$-linear map $f: E^{k} \rightarrow \mathbb{K}$ and the map $\bar{f}: E^{\otimes k} \rightarrow \mathbb{K}$, where $\otimes$ is the algebraic tensor product, which is linear and defined as follows: if $\chi=\sum_{j} \chi_{1}^{j} \otimes \cdots \otimes \chi_{k}^{j}$ is a finite sum of tensor products, then

$$
\bar{f}(\chi)=\sum_{j} f\left(\chi_{1}^{j}, \ldots, \chi_{k}^{j}\right)
$$

Let us give a topological version of this lemma, using the projective topology. We recall the definition of a family of seminorms defining the projective topology on tensor powers of locally convex spaces following (Ref. 71, p. 23). For arbitrary seminorms $p_{1}, \ldots, p_{k}$ on $E$, there exists a seminorm $p_{1} \otimes \cdots \otimes p_{k}$ on $E^{\otimes k}$ defined for every $\psi \in E^{\otimes k}$ by

$$
p_{1} \otimes \cdots \otimes p_{k}(\psi)=\inf \sum_{n} p_{1}\left(e_{1, n}\right) \ldots p_{k}\left(e_{k, n}\right),
$$

where the infimum is taken over the representations of $\psi$ as finite sums: $\psi=\sum_{n} e_{1, n} \otimes \cdots \otimes e_{k, n}$. Following Köthe (Ref. 72, p. 178), one can prove that $p_{1} \otimes \cdots \otimes p_{k}$ is the largest seminorm on $E^{\otimes k}$ such that

$$
p\left(x_{1} \otimes \cdots \otimes x_{k}\right)=p_{1}\left(x_{1}\right) \ldots p_{k}\left(x_{k}\right),
$$

for all $x_{1}, \ldots, x_{k}$ in $E$. More precisely, if $p$ is a seminorm on $E^{\otimes k}$ satisfying Eq. (8), then $p(X) \leqslant\left(p_{1}\right.$ $\left.\otimes \cdots \otimes p_{k}\right)(X)$ for every $X \in E^{\otimes k}$.

The projective topology on $E^{\otimes k}$ is defined by the family of seminorms $p_{1} \otimes \cdots \otimes p_{k}$ where each $p_{i}$ runs over a family of seminorms defining the topology of $E$ (Ref. 71, p. 24).

When $E$ is Fréchet, its topology is defined by a countable family of seminorms and it follows that the family of seminorms $p_{1} \otimes \cdots \otimes p_{k}$ on $E^{\otimes k}$ is countable. Hence they can be used to construct a metric on $E^{\otimes k}$ which defines the same topology as the projective topology and $E^{\hat{\otimes}_{\pi} k}$ is defined as the completion of $E^{\otimes k}$ relative to this metric or equivalently with respect to the projective topology. A fundamental property of the projective topology is that $f: E^{k} \rightarrow \mathbb{K}$ is (jointly) continuous if and only if $\bar{f}: E^{\otimes k} \rightarrow \mathbb{K}$ and still defined by Eq. (7) is continuous with respect to the projective topology (Ref. 73, p. I-50). Then $\bar{f}$ extends uniquely to a continuous map (still denoted by $\bar{f}$ ) on the completed tensor product $E^{\hat{\otimes}_{\pi} k}$ (Ref. 61, p. III.15).

If $E=C^{\infty}(M)$, then $E^{\hat{\otimes}_{\pi} k}=C^{\infty}\left(M^{k}\right)$ (Ref. 31, p. 530), and $\bar{f}$ becomes a compactly supported distribution on $M^{k}$. More generally, if $E=\Gamma(M, B)$, then $E$ is Fréchet nuclear and $E^{\hat{\otimes}_{\pi} k}=\Gamma\left(M^{k}, \mathcal{E}^{\boxplus k}\right)$ (Ref. 74, p. 72). Thus, $\bar{f}$ becomes a compactly supported distributional section on $M^{k}$. If $f=D^{k} F_{\varphi}$, we denote $\bar{f}$ by $F_{\varphi}^{(k)}$.

Recall that if $U$ is an open subset of a Hausdorff locally convex space $E$, a map $F: U \rightarrow \mathbb{K}$ is smooth if and only if every $D^{k} F$ is continuous from $U \times E^{k}$ to $\mathbb{K}$. According to the previous discussion, continuity on $E^{k}$ is equivalent to continuity on $E^{\hat{\otimes}_{\pi} k}$. Therefore, it is natural to wonder when joint continuity on $U \times E^{k}$ is equivalent to joint continuity on $U \times E^{\hat{\otimes}_{\pi} k}$. This is the subject of the next paragraphs.

We now prove an equicontinuity lemma.

Lemma III.6. Let $E$ be a Fréchet space, $U$ be open in $E$, and $F: U \times E^{k} \mapsto \mathbb{K}$ be a continuous map, multilinear in the last $k$ variables. Then for every $\varphi_{0} \in U$, there exist a neighborhood $V$ of $\varphi_{0}$, a seminorm $q$ of $E^{\hat{\otimes}_{\pi} k}$, and a constant $C>0$ such that

$$
\forall \varphi \in V, \forall \psi \in E^{\hat{\otimes}_{\pi} k},\left|\bar{F}(\varphi, \psi)-\bar{F}\left(\varphi_{0}, \psi\right)\right| \leqslant C q(\psi) .
$$

Proof. By continuity of $F: U \times E^{k} \mapsto \mathbb{K}$, for every $\epsilon>0$, there exist a neighborhood $V$ of $\varphi_{0}$ and neighborhoods $U_{1}, \ldots, U_{k}$ of zero such that $\varphi \in V$ and $e_{i} \in U_{i}$ for $i=1, \ldots, k$ imply $\mid F\left(\varphi, e_{1}\right.$, $\left.\ldots, e_{k}\right) \mid \leq \epsilon$. Since $E$ is locally convex, there are continuous seminorms $p_{1}, \ldots, p_{k}$ on $E$ and strictly 
positive numbers $\eta_{1}, \ldots, \eta_{k}$ such that $e_{i} \in U_{i}$ if $p_{i}\left(e_{i}\right) \leq \eta_{i}$. Consider now arbitrary elements $e_{1}, \ldots$, $e_{k}$ of $E$ such that $p_{i}\left(e_{i}\right) \neq 0$. Then, if $f_{i}=e_{i} \eta_{i} / p_{i}\left(e_{i}\right)$, we have $p_{i}\left(f_{i}\right) \leq \eta_{i}$. Thus, $\left|F\left(\varphi, f_{1}, \ldots, f_{k}\right)\right|<\epsilon$ and, by multilinearity, $\left|F\left(\varphi, e_{1}, \ldots, e_{k}\right)\right|<M p_{1}\left(e_{1}\right) \ldots p_{k}\left(e_{k}\right)$ where $M=\epsilon /\left(\eta_{1}, \ldots, \eta_{k}\right)$. The argument in the proof of Proposition III.4 shows that $\left|F\left(\varphi, e_{1}, \ldots, e_{k}\right)\right|=0$ if $p_{i}\left(e_{i}\right)=0$. Therefore, for every $\varphi$ $\in V$ and every $e_{1}, \ldots, e_{k}$ in $E,\left|F\left(\varphi, e_{1}, \ldots, e_{k}\right)\right| \leq M p_{1}\left(e_{1}\right) \ldots p_{k}\left(e_{k}\right)$. By defining $C=2 M$, we obtain for every $\varphi \in V$ and every $\left(e_{1}, \ldots, e_{k}\right) \in E^{k}$

$$
\left|F\left(\varphi, e_{1}, \ldots, e_{k}\right)-F\left(\varphi_{0}, e_{1}, \ldots, e_{k}\right)\right| \leqslant C p_{1}\left(e_{1}\right) \ldots p_{k}\left(e_{k}\right) .
$$

By definition of $\bar{F}$, for all $(\varphi, \psi) \in V \times E^{\otimes k}$,

$$
\begin{aligned}
\left|\bar{F}(\varphi, \psi)-\bar{F}\left(\varphi_{0}, \psi\right)\right| \leqslant & \sum_{n} \mid F\left(\varphi, e_{1, n}, \ldots, e_{k, n}\right) \\
& -F\left(\varphi_{0}, e_{1, n}, \ldots, e_{k, n}\right) \mid \\
\leqslant & C \sum_{n} p_{1}\left(e_{1, n}\right) \ldots p_{k}\left(e_{k, n}\right),
\end{aligned}
$$

for all representations of $\psi$ as finite sum $\psi=\sum_{n} e_{1, n} \otimes \cdots \otimes e_{k, n}$. Taking the infimum over such representations yields the estimate

$$
\forall(\varphi, \psi) \in V \times E^{\otimes k},\left|\bar{F}(\varphi, \psi)-\bar{F}\left(\varphi_{0}, \psi\right)\right| \leqslant C q(\psi),
$$

for the seminorm $q=p_{1} \otimes \cdots \otimes p_{k}$ on $E^{\otimes_{\pi} k}$ and the above inequality extends to any $\psi$ of $E^{\hat{\otimes}_{\pi} k}$ since, in a Fréchet space, $\psi$ can be approximated by a convergent sequence of elements in $E^{\otimes k}$ by the density of $E^{\otimes k}$ in $E^{\hat{\otimes}_{\pi} k}$ and by the continuity of the seminorm $p_{1} \otimes \cdots \otimes p_{k}$ for the topology of $E^{\hat{\otimes}_{\pi} k}$.

Another way to state the previous result is to say that the family of linear maps $\{F(\varphi, \cdot), \varphi \in V\}$, is equicontinuous (Ref. 61, p. II.6).

\section{Proof of the main result}

We are now ready to prove

Proposition III.7. Let $E$ be a Fréchet space and $U \subset E$ be an open subset. Then $F: U \times E^{k} \mapsto \mathbb{K}$, multilinear in the last $k$ variables, is jointly continuous if and only if the corresponding map $\bar{F}$ : $U \times E^{\hat{\otimes}_{\pi} k} \rightarrow \mathbb{K}$ is jointly continuous.

Proof. One direction of this theorem is straightforward and holds if $E$ is any locally convex space. Indeed, by definition of the projective tensor product, the canonical multilinear mapping $E^{k} \rightarrow E^{\hat{\otimes} k}$ is continuous (Ref. 73, p. I-50). Therefore, if $\bar{F}$ is continuous on $U \times E^{\hat{\otimes} k}$, then, by composition with the canonical multilinear mapping, $F$ is continuous on $U \times E^{k}$.

Let us prove that the continuity of $F$ implies the continuity of $\bar{F}$. According to Lemma II.4, we have to show that, for every $\varphi_{0} \in U$ and every $\varepsilon>0$, there exist a finite number of continuous seminorms $q_{i}$ on $E^{\hat{\otimes}_{\pi} k}$, a neighborhood $V$ of $\varphi_{0}$, and $\eta_{i}>0$ such that if $\varphi$ belongs to $V$ and $\psi \in E^{\hat{\otimes}_{\pi} k}$ satisfies $q_{i}\left(\psi-\psi_{0}\right) \leqslant \eta_{i}$, then $\left|\bar{F}(\varphi, \psi)-\bar{F}\left(\varphi_{0}, \psi_{0}\right)\right| \leqslant \varepsilon$.

In order to bound $\bar{F}(\varphi, \psi)-\bar{F}\left(\varphi_{0}, \psi_{0}\right)$, we cut it into three parts:

$$
\begin{aligned}
\bar{F}(\varphi, \psi)-\bar{F}\left(\varphi_{0}, \psi_{0}\right) & =\bar{F}\left(\varphi, \psi_{k}\right)-\bar{F}\left(\varphi_{0}, \psi_{k}\right) \\
& +\bar{F}\left(\varphi, \psi-\psi_{k}\right)-\bar{F}\left(\varphi_{0}, \psi-\psi_{k}\right) \\
& +\bar{F}\left(\varphi_{0}, \psi\right)-\bar{F}\left(\varphi_{0}, \psi_{0}\right),
\end{aligned}
$$

where $\psi_{k}$ is some element of the algebraic tensor product $E^{\otimes k}$ close enough to $\psi_{0}$ that we choose now. The equicontinuity lemma III.6 yields a neighborhood $V_{2}$ of $\varphi_{0}$, a constant $C>0$, and a continuous seminorm $q_{2}$ on $E^{\hat{\otimes}_{\pi} k}$ so that

$$
\forall \varphi \in V_{2}, \forall \psi \in E^{\hat{\otimes}_{\pi} k},\left|\bar{F}(\varphi, \psi)-\bar{F}\left(\varphi_{0}, \psi\right)\right| \leqslant C q_{2}(\psi) .
$$


Now we use the fact that the algebraic tensor product $E^{\otimes k}$ is everywhere dense in $E^{\hat{\otimes}_{\pi} k}$, hence there is some element $\psi_{k}$ in the algebraic tensor product $E^{\otimes k}$ such that $q_{2}\left(\psi_{0}-\psi_{k}\right) \leqslant \eta_{2}$ with $\eta_{2}:=\frac{\varepsilon}{6 C}$.

Now that $\psi_{k}$ is chosen, we can bound the second term of the sum (11), namely, $\bar{F}\left(\varphi, \psi-\psi_{k}\right)-$ $\bar{F}\left(\varphi_{0}, \psi-\psi_{k}\right)$. From the previous relation, for every $\varphi \in V_{2}$ and every $\psi$ such that $q_{2}\left(\psi-\psi_{0}\right) \leq \eta_{2}$, the triangle inequality for $q_{2}\left(\psi-\psi_{k}\right)$ gives us

$$
\left|\bar{F}\left(\varphi, \psi-\psi_{k}\right)-\bar{F}\left(\varphi_{0}, \psi-\psi_{k}\right)\right| \leqslant C\left(q_{2}\left(\psi-\psi_{0}\right)+q_{2}\left(\psi_{0}-\psi_{k}\right)\right) \leqslant \frac{\varepsilon}{3} .
$$

We continue by bounding the last term $\bar{F}\left(\varphi_{0}, \psi\right)-\bar{F}\left(\varphi_{0}, \psi_{0}\right)$ in the sum (11). Since $\varphi_{0}$ is fixed, the map $\psi \mapsto \bar{F}\left(\varphi_{0}, \psi\right)$ is continuous in $\psi$ because, since $\bar{F}\left(\varphi_{0}, \cdot\right)$ is continuous on $E^{\otimes_{\pi} k}$, its extension to the completion $E^{\hat{\otimes}_{\pi} k}$, also denoted by $\bar{F}\left(\varphi_{0}, \cdot\right)$, is continuous. It follows that there is some seminorm $q_{1}$ of $E^{\hat{\otimes}_{\pi} k}$ and a number $\eta_{1}>0$ such that if $\psi \in U$ satisfies $q_{1}\left(\psi-\psi_{0}\right) \leqslant \eta_{1}$, then

$$
\left|\bar{F}\left(\varphi_{0}, \psi\right)-\bar{F}\left(\varphi_{0}, \psi_{0}\right)\right| \leqslant \frac{\varepsilon}{3} .
$$

To bound the first term $\bar{F}\left(\varphi, \psi_{k}\right)-\bar{F}\left(\varphi_{0}, \psi_{k}\right)$ in the sum (11), we use the fact that $\psi_{k} \in E^{\otimes k}$. Thus, $\psi_{k}=\sum_{j=1}^{p}\left(e_{1, j} \otimes \cdots \otimes e_{k, j}\right)$ for some $\left(e_{1, j}, \ldots, e_{k, j}\right) \in E^{k}$. By definition of $\bar{F}$,

$$
\bar{F}\left(\varphi, \psi_{k}\right)-\bar{F}\left(\varphi_{0}, \psi_{k}\right)=\sum_{j=1}^{p} F\left(\varphi, e_{1, j}, \ldots, e_{k, j}\right)-F\left(\varphi_{0}, e_{1, j}, \ldots, e_{k, j}\right) .
$$

By continuity of $F$ in the first factor, the finite $\operatorname{sum} \sum_{j=1}^{p} F\left(\varphi, e_{1, j}, \ldots, e_{k, j}\right)$ is continuous in $\varphi$ and there is some neighborhood $V_{3}$ of $\varphi_{0}$ such that for all $\varphi \in V_{3}$ the following bound

$$
\left|\sum_{j=1}^{p} F\left(\varphi, e_{1, j}, \ldots, e_{k, j}\right)-F\left(\varphi_{0}, e_{1, j}, \ldots, e_{k, j}\right)\right| \leqslant \frac{\varepsilon}{3}
$$

holds true.

Finally we found some neighborhood $V=V_{2} \cap V_{3}$ of $\varphi_{0}$, two seminorms $q_{1}$ and $q_{2}$ of $E^{\hat{\otimes}_{\pi} k}$, and two numbers $\eta_{1}>0$ and $\eta_{2}=\epsilon / 6 C$ such that $q_{1}\left(\psi-\psi_{0}\right)<\eta_{1}$ and $q_{2}\left(\psi-\psi_{0}\right)<\eta_{2}$ imply

$$
\left|\bar{F}(\varphi, \psi)-\bar{F}\left(\varphi_{0}, \psi_{0}\right)\right| \leqslant \varepsilon
$$

The proposition is proved.

Now we can specialize our result to the space of smooth sections of vector bundles. We recall a fundamental result on the projective tensor product of sections (Ref. 74, p. 72):

Proposition III.8. Let $\Gamma(M, B)$ be the space of smooth sections of some smooth finite rank vector bundle $B \rightarrow$ M on a manifold $M$. Then $\Gamma(M, B)^{\hat{\otimes}_{\pi} k}=\Gamma\left(M^{k}, B^{\bowtie k}\right)$.

Note that we could remove the index $\pi$ in $\hat{\otimes}_{\pi}$ because we saw that $\Gamma(M, B)$ is nuclear. If we specialize Proposition III.7 to sections of vector bundles (which is a Fréchet space), we obtain

Theorem III.9. Let $E=\Gamma(M, B)$ be the space of smooth sections of some smooth finite rank vector bundle $B \rightarrow M$. Then $F: U \times E^{k} \rightarrow \mathbb{K}$ multilinear in the last $k$ variables is jointly continuous if and only if the corresponding map $\bar{F}: U \times \Gamma\left(M^{k}, B^{\otimes k}\right) \rightarrow \mathbb{K}$ is jointly continuous.

Proof. The proof is an immediate consequence of the fact that $E^{\hat{\otimes}_{\pi} k}=\Gamma\left(M^{k}, B^{\bowtie k}\right)$ and Proposition III.7.

The definition of a Bastiani smooth functional implies the following corollary: 
Theorem III.10. Let $E=\Gamma(M, B)$ be the space of smooth sections of some smooth finite rank vector bundle $B \rightarrow M$. A map $F: U \rightarrow \mathbb{K}$, where $U$ is open in $E$, is Bastiani smooth if and only if the maps $F^{(k)}: U \times \Gamma\left(M^{k}, B^{\otimes k}\right) \rightarrow \mathbb{K}$ are (jointly) continuous for every $k \geq 1$.

To interpret Theorem III.7 in terms of distributional kernels, let $B \rightarrow M$ denote a smooth vector bundle of finite rank over a manifold $M$ equipped with a fixed density $|d x|$ and $B^{*} \rightarrow M$ denote the corresponding dual bundle. Recall that $\Gamma(M, B)^{\prime} \simeq \Gamma\left(M, B^{*}\right) \otimes_{C^{\infty}(M)} \mathcal{E}^{\prime}(M),{ }^{75}$ where $\Gamma\left(M, B^{*}\right) \otimes_{C^{\infty}(M)} \mathcal{E}^{\prime}(M)$ denotes the compactly supported distributional sections of the dual bundle $B^{*}$. In global analysis, to every continuous linear map $L: \Gamma(M, B) \rightarrow \Gamma(M, B)^{\prime}$, we associate the continuous bilinear map $B:\left(\varphi_{1}, \varphi_{2}\right) \in \Gamma(M, B)^{2} \mapsto\left\langle\varphi_{1}, L \varphi_{2}\right\rangle \in \mathbb{K}$ where the pairing is understood as a pairing between a smooth function and a distribution once the smooth density on $M$ is fixed.

The usual kernel theorem of the theory of distributions states that a bilinear map can be represented by a distribution $K_{L} \in \mathcal{E}^{\prime}(M \times M) \otimes_{C^{\infty}\left(M^{2}\right)} \Gamma\left(M^{2}, B^{*} \otimes B^{*}\right)$ living on configuration space $M^{2}$ such that, for every $\left(\varphi_{1}, \varphi_{2}\right) \in \Gamma(M, B)^{2}$,

$$
\left\langle K_{L}, \varphi_{1} \otimes \varphi_{2}\right\rangle_{\Gamma_{2}^{\prime}, \Gamma_{2}}=\left\langle\varphi_{1}, L \varphi_{2}\right\rangle_{\Gamma(M, B), \Gamma(M, B)^{\prime}},
$$

where $\Gamma_{2}=\Gamma\left(M^{2}, B \otimes B\right)$. Theorem III.7 generalizes the kernel theorem by using multilinear maps instead of the bilinear ones and by considering that these multilinear maps depend continuously and non-linearly on a parameter $\varphi$.

\section{Order of distributions}

If $F$ is a Bastiani smooth map from an open subset $U$ of $E=C^{\infty}(M)$ to $\mathbb{K}$, then, for every $\varphi \in$ $U, D^{k} F_{\varphi}$ is a compactly supported distribution. Therefore, the order of $F_{\varphi}^{(k)}$ is finite (Ref. 27, p. 88). For some applications, for example, to local functionals, it is important to require the order of $F_{\varphi}^{(k)}$ to be locally bounded.

Proposition III.11. Let $E=C^{\infty}(M)$ and $F: E \rightarrow \mathbb{K}$ be a smooth functional on an open subset $U$ of $E$. Then, for every $\varphi_{0} \in U$ and every integer $k$, there is a neighborhood $V$ of $\varphi_{0}$, an integer $m$, and a compact $K \subset M^{k}$ such that, for every $\varphi \in V$, the order of $F_{\varphi}^{(k)}$ is smaller than $m$ and $F_{\varphi}^{(k)}$ is supported in $K$.

Proof. According to Lemma III.6, for every $\varphi_{0}$ in $U$, there is a neighborhood $V$ of $\varphi_{0}$, a constant $C$, and a seminorm $\pi_{n, K}$ of $C^{\infty}(M)$ such that

$$
\left|F_{\varphi}^{(k)}(\psi)-F_{\varphi_{0}}^{(k)}(\psi)\right| \leqslant C \pi_{n, K}(\psi) .
$$

This means that the order of $F_{\varphi}^{(k)}-F_{\varphi_{0}}^{(k)}$ is bounded by $n$ (Ref. 27, p. 64), and the order of $F_{\varphi}^{(k)}$ is bounded by $n$ plus the order of $F_{\varphi_{0}}^{(k)}$. Moreover, if supp $\psi \cap K=\emptyset$, then $\pi_{n, K}(\psi)=0$ and $F_{\varphi}^{(k)}(\psi)-F_{\varphi_{0}}^{(k)}(\psi)=0$. This means that the support of $F_{\varphi}^{(k)}-F_{\varphi_{0}}^{(k)}$ is contained in $K$ and the support of $F_{\varphi}^{(k)}(\psi)$ is contained in the compact $K \cup \operatorname{supp} F_{\varphi_{0}}^{(k)}$.

Note also that, in general, the order of the distributions is not bounded on $U$.

Lemma III.12. Let $g \in \mathcal{D}(\mathbb{R})$ and $\left(\chi_{n}\right)_{n \in \mathbb{Z}}$ be a sequence of functions such that $\chi_{n} \in \mathcal{D}([n-1, n+$ 1]) and $\sum_{n \in \mathbb{Z}} \chi_{n}=1$. Then, the functional

$$
F(\varphi)=\sum_{n=-\infty}^{\infty} \int_{\mathbb{R}} \chi_{n}(\varphi(x)) \frac{d^{|n|} \varphi}{d x^{|n|}}(x) g(x) d x
$$

is Bastiani smooth on $C^{\infty}(\mathbb{R})$ but the order of $F^{(k)}$ is not bounded on $C^{\infty}(\mathbb{R})$.

Proof. The functional $F$ is smooth because, for every $\varphi_{0} \in C^{\infty}(\mathbb{R})$, we can define a neighborhood of $\varphi_{0}$ by $V=\left\{\varphi ; \pi_{0, K}\left(\varphi-\varphi_{0}\right)<\epsilon\right\}$, where $K$ is a compact neighborhood of the support of $g$. Let $N$ 
be the smallest integer strictly greater than $\pi_{0, K}\left(\varphi_{0}\right)+\epsilon$. Then, $-N<\varphi(x)<N$ for every $\varphi \in V$ and every $x \in K$ and

$$
F(\varphi)=\sum_{n=-N-1}^{N+1} \int \chi_{n}(\varphi(x)) \varphi^{(|n|)}(x) g(x) d x
$$

is a finite sum of smooth functionals.

However, the order of

$$
F_{\varphi}^{(1)}(\psi)=\sum_{n=-\infty}^{\infty} \int\left(\chi_{n}(\varphi(x)) \psi^{(|n|)}(x)+\chi_{n}^{\prime}(\varphi(x)) \psi^{(1)}(x) \varphi^{(|n|)}(x)\right) g(x) d x
$$

is not bounded on $C^{\infty}(\mathbb{R})$. Indeed, for any positive integer $n$, we can find a smooth function $\varphi$ such that $\chi_{n}(\varphi(x)) g(x) \neq 0$ for some $x \in \operatorname{supp} g$. Since $F_{\varphi}^{(1)}(\psi)$ contains a factor $\psi^{(n)}(x)$, it is at least of order $n$.

\section{Derivatives as smooth functionals}

In Sec. IV, we equip several spaces of functionals with a topology. As a warm-up exercise, we show here that the maps $F^{(k)}$ are smooth functionals from $C^{\infty}(M)$ to $\mathcal{E}^{\prime}\left(M^{k}\right)$.

We adapt to the case of functionals the general result given in item 4 of Proposition II.13 stating that if $F$ is a smooth functional on $U$, then $D^{k} F$ is a Bastiani smooth map from $U$ to $L\left(E^{k}, \mathbb{K}\right)$. We need to identify the topology of $L\left(E^{k}, \mathbb{K}\right)$ used by Bastiani. Let us start with $L(E, \mathbb{K})$. Bastiani furnishes $E$ with the topology of convergence on all compact sets of $E$. In other words, the seminorms that define the topology of $L(E, \mathbb{K})$ are $p_{C}(u)=\sup _{f \in C}|\langle u, f\rangle|$, where $C$ runs over the compact subsets of $C^{\infty}(M)$. Since $C^{\infty}(M)$ is a Montel space (Ref. 30, p. 239), the topology of uniform convergence on compact sets is the same as the strong topology (Ref. 30, p. 235). This means that $L(E, \mathbb{K})$ is the space $\mathcal{E}^{\prime}(M)$ of compactly supported distributions with its usual topology. Similarly, $L\left(E^{k}, \mathbb{K}\right)$ can be identified to a subset of $\mathcal{E}^{\prime}\left(M^{k}\right)$ with its usual topology. We just obtained the following result:

Proposition III.13. Let $U$ be an open subset of $C^{\infty}(M)$ and $F: U \rightarrow \mathbb{K}$ be a Bastiani smooth functional. Then, for every integer $k$, the map $F^{(k)}: U \rightarrow \mathcal{E}^{\prime}\left(M^{k}\right)$ is smooth in the sense of Bastiani.

\section{TOPOLOGIES ON SPACES OF FUNCTIONALS}

We need to define a topology on the various spaces of functionals used in quantum field theory. The general idea is to define seminorms on $F$ and its derivatives $F^{(k)}$. The topology proposed by Brunetti, Dütsch, and Fredenhagen ${ }^{17}$ is the initial topology of all the maps $F \rightarrow F_{\varphi}^{(k)}$, where each $F_{\varphi}^{(k)}$ belongs to a nuclear space determined by a wavefront set condition. This topology is nuclear, but the absence of a control of the dependence on $\varphi$ makes it generally not complete. We then describe Bastiani's topology, which is complete but has two drawbacks: it does not take wavefront set conditions into account and it is generally not nuclear. Finally we shall describe the family of topologies proposed by Dabrowski ${ }^{21}$ which are both nuclear and complete.

\section{A. Bastiani's topology}

Bastiani defines several topologies on the space of Bastiani smooth maps between two locally convex spaces (Ref. 41, p. 65). For the case of functionals, we consider the topology defined by the following seminorms:

$$
\begin{aligned}
p_{C_{0}}(F) & =\sup _{\varphi \in C_{0}}|F(\varphi)|, \\
p_{C_{0}, C}(F) & =\sup _{\left(\varphi, h_{1}, \ldots, h_{k}\right) \in C_{0} \times C}\left|D^{k} F_{\varphi}\left(h_{1}, \ldots, h_{k}\right)\right|,
\end{aligned}
$$

where $C=C_{1} \times \cdots \times C_{k}$ and $C_{i}$ runs over the compact sets of $\Gamma(M, B)$. By using Bastiani's results (Ref. 41, p. 66), we obtain 
Proposition IV.1. Let $B \stackrel{\pi}{\rightarrow} M$ be a finite rank vector bundle over the manifold $M$ and $\Gamma(M, B)$ be the space of smooth sections of $B$. Then, with the seminorms defined above, the space of smooth functionals on $\Gamma(M, B)$ is a complete locally convex space.

A similar topology was used by Glöckner (Ref. 76, p. 367) and Wockel (Ref. 77, p. 29 and Ref. 78, p. 12).

\section{B. Nuclear and complete topologies}

Quantum field theory uses different spaces of functionals defined by conditions on the wave front set of $F_{\varphi}^{(k)}$. Recall that the wave front set describes the points and the directions of singularity of a distribution. ${ }^{79}$ Dabrowski ${ }^{21}$ recently described nuclear and complete topologies for spaces of functionals with wave front set conditions. We present some of his topologies for several common spaces of functionals.

Dabrowski's definition differs from Bastiani in two respects. To describe the first difference, recall that, according to Proposition III.13, if $F: U \rightarrow \mathbb{R}$ is a smooth functional, then the derivatives $F^{(k)}: U \rightarrow \mathcal{E}^{\prime}\left(M^{k}\right)$ are smooth functionals. To add the wave front set conditions, Dabrowski requires $F^{(k)}$ to be smooth from $U$ to $\mathcal{E}_{\Gamma_{k}}^{\prime}\left(M^{k}\right)$, which is the space of compactly supported distributions whose wave front sets are included in $\Gamma_{k}$, a cone in $T^{*} M^{k}$. In fact, Dabrowski supplements this definition with a more refined wave front set (the dual wave front set) which enables him to equip $\mathcal{E}_{\Gamma_{k}}^{\prime}\left(M^{k}\right)$ with a Montel, complete, ultrabornological, and nuclear topology. He also considers support conditions which are different from compact.

To describe the second difference, recall that Bastiani's topology gives a locally convex space that is complete. However it is generally not nuclear. This is due to a theorem by Colombeau and Meise ${ }^{80}$ which says, broadly speaking, that a function space over a Fréchet space cannot be nuclear for the topology of convergence over some balanced, convex, compact sets of infinite dimension. To avoid that problem, the variable $\varphi$ is made to run over finite dimensional compact sets. More precisely, Dabrowski considers compact sets in $\mathbb{R}^{m}$ for any finite value of $m$ and smooth maps $f$ from $\mathbb{R}^{m}$ to an open subset of $C^{\infty}(M)$. He defines two families of seminorms:

$$
\begin{aligned}
p_{f, K}(F) & =\sup _{\varphi \in f(K)}|F(\varphi)|, \\
p_{n, f, K, C}(F) & =\sup _{\varphi \in f(K)} \sup _{v \in C}\left|\left\langle F_{\varphi}^{(n)}, v\right\rangle\right|,
\end{aligned}
$$

where $K$ is a compact subset of $\mathbb{R}^{m}$ for some $m$ and $C$ is an equicontinuous subset of the dual of the space of distributions to which $F_{\varphi}^{(n)}$ belong. Dabrowski proved that, with this family of seminorms, the space of functionals $\mathrm{F}$ is a complete locally convex nuclear space. ${ }^{22}$

We describe now several types of functionals that have been used in the literature and we specify more precisely their topologies.

\section{The regular functionals}

A polynomial functional of the form

$$
F(\varphi)=\sum_{n} \int_{M^{n}} d x_{1} \ldots d x_{n} f_{n}\left(x_{1}, \ldots, x_{n}\right) \varphi\left(x_{1}\right) \ldots \varphi\left(x_{n}\right),
$$

where the sum over $n$ is finite and $f_{n} \in \mathcal{D}\left(M^{n}\right)$, is called a regular functional ${ }^{81}$ because all its derivatives are smooth functions, ${ }^{82}$ i.e., the wave front set of $F_{\varphi}^{(k)}$ is empty. More generally, we define the space $\mathrm{F}_{\text {reg }}(M)$ of regular functionals to be the set of Bastiani smooth functionals $F$ such that $\operatorname{WF}\left(F^{(n)}\right)=\emptyset$ for every $n>0$. Thus, $F^{(n)} \in \mathcal{E}_{\emptyset}^{\prime}\left(M^{n}\right)=\mathcal{D}\left(M^{n}\right)$ and the sets $C$ in Eq. (13) are the equicontinuous sets of $\mathcal{D}^{\prime}\left(M^{n}\right)$. By a general theorem (Ref. 30, p. 200), the topology of uniform convergence on the equicontinuous sets of $\mathcal{D}^{\prime}\left(M^{n}\right)$ is equivalent to the topology given by the seminorms of its dual $\mathcal{D}\left(M^{n}\right)$. In other words, the topology of $\mathrm{F}_{\text {reg }}(M)$ is defined by the 
seminorms $^{22}$

$$
\begin{gathered}
p_{f, K}(F)=\sup _{\varphi \in f(K)}|F(\varphi)|, \\
p_{n, f, K, \alpha}(F)=\sup _{\varphi \in f(K)} p_{\alpha, n}\left(F_{\varphi}^{(n)}\right),
\end{gathered}
$$

where $p_{\alpha, n}$ runs over a defining family of seminorms of $\mathcal{D}\left(M^{n}\right) .{ }^{83}$ With this topology, $\mathrm{F}_{\text {reg }}(M)$ is nuclear and complete.

Note that the tensor product of elements of $\mathcal{D}\left(M^{m}\right)$ with elements of $\mathcal{D}\left(M^{n}\right)$ is not continuous in $\mathcal{D}\left(M^{m+n}\right) .{ }^{84}$ Thus, the product in $\mathrm{F}_{\text {reg }}(M)$ is hypocontinuous but not continuous.

\section{The microcausal functionals}

It is possible to describe quantum field theory (up to renormalization) as the deformation quantization of classical field theory. ${ }^{26}$ For the deformation quantization of the product $F G$ of two functionals to first order in $\hbar$, we need to evaluate $\left\langle D F_{\varphi} \otimes D G_{\varphi}, \Delta_{+}\right\rangle$, where $\Delta_{+}$is a singular distribution (the Wightman propagator) and $\langle\cdot, \cdot\rangle$ is an extension of the duality pairing between distributions and test functions. ${ }^{85}$ For this pairing to be meaningful to all orders in $\hbar$, the wave front set of $\Delta_{+}$imposes that the wave front set of $D_{\varphi}^{(k)}$ must not meet the cone $\Gamma_{k}$ defined as follows. ${ }^{26}$

Let $M$ be a Lorentzian manifold with pseudo-metric $g$. Let $V_{x}^{+}$(respectively, $V_{x}^{-}$) be the set of $(x ; \xi) \in T_{x}^{*} M$ such that $g^{\mu v}(x) \xi_{\mu} \xi_{v} \geq 0$ and $\xi_{0} \geq 0$ (respectively, $\xi_{0} \leq 0$ ), where we assume that $g^{00}$ $>0$. We define the closed cone

$$
\begin{aligned}
\Gamma_{n}= & \left\{\left(x_{1}, \ldots, x_{n} ; \xi_{1}, \ldots, \xi_{n}\right) \in \dot{T}^{*} M^{n} ;\left(\xi_{1}, \ldots, \xi_{n}\right) \in\right. \\
& \left.\left(V_{x_{1}}^{+} \times \cdots \times V_{x_{n}}^{+}\right) \cup\left(V_{x_{1}}^{-} \times \cdots \times V_{x_{n}}^{-}\right)\right\},
\end{aligned}
$$

where $\dot{T}^{*} M^{n}$ is the cotangent bundle $T^{*} M^{n}$ without its zero section. The space $\mathrm{F}_{\mathrm{mc}}$ of microcausal functionals was originally defined as the set of Bastiani smooth functionals such that $F_{\varphi}^{(n)} \in \mathcal{E}_{\Xi_{n}}^{\prime}\left(M^{n}\right)$ for every $\varphi$, where $\Xi_{n}=\dot{T}^{*} M^{n} \backslash \Gamma_{n}$ is an open cone. ${ }^{16,17,20,26,81,86,87}$

However, the space $\mathcal{E}_{\Xi_{n}}^{\prime}\left(M^{n}\right)$ being not even sequentially complete, ${ }^{85}$ it is not suitable to define a complete space of functionals. Therefore, Dabrowski defines the space $F_{\mathrm{mc}}$ of microcausal functionals to be the set of Bastiani smooth functionals such that $F_{\varphi}^{(n)} \in \mathcal{E}_{\Xi_{n}, \bar{\Xi}_{n}}^{\prime}\left(M^{n}\right)$, which is the completion of $\mathcal{E}_{\Xi_{n}}^{\prime}\left(M^{n}\right)$. Dabrowski proved that $\mathcal{E}_{\Xi_{n}, \bar{\Xi}_{n}}^{\prime}\left(M^{n}\right)$ is the set of compactly supported distributions $u \in \mathcal{E}^{\prime}\left(M^{n}\right)$ such that the dual wavefront set of $u$ is in $\Xi_{n}$ and the wavefront set of $u$ is in its closure $\bar{\Xi}_{n}$ (see Ref. 21 for a precise definition of these concepts and of the topology). This completion is not only complete but even Montel and nuclear. ${ }^{21}$ According to the general results of Ref. 21, the sets $C$ are now equicontinuous sets of the bornologification of the normal topology of $\mathcal{D}_{\Gamma_{n}}^{\prime}$. However, it was shown ${ }^{21}$ that these equicontinuous sets are the same as the bounded sets of $\mathcal{D}_{\Gamma_{n}}^{\prime}$ with its normal topology. Therefore, the sets $C$ are the well-known bounded sets of $\mathcal{D}_{\Gamma_{n}}^{\prime}{ }^{85}$

With this topology, the space $F_{\mathrm{mc}}$ is a complete nuclear algebra with hypocontinuous product.

\section{E. Local functionals}

As discussed in the Introduction, local functionals are the basic building block (Lagrangian) of quantum field theory. We shall see that local functionals are a closed subset of the set of smooth functionals such that $F_{\varphi}^{(1)}$ can be identified with an element of $\mathcal{D}(M)$ that we denote by $\nabla F_{\varphi}$ and the wave front set of $F_{\varphi}^{(k)}$ is included in the conormal $C_{k}$ of $D_{k}=\left\{\left(x_{1}, \ldots, x_{k}\right) \in M^{k} ; x_{1}=\cdots=x_{k}\right\}$. Recall that the conormal of $D_{k}$ is the set of $\left(x_{1}, \ldots, x_{k} ; \xi_{1}, \ldots, \xi_{k}\right) \in T^{*} M^{k}$ such that $x_{1}=\cdots=x_{k}$ and $\xi_{1}$ $+\cdots+\xi_{k}=0$.

Since the additivity property (defined in Sec. V C) of local functionals complicates the matter, we follow Dabrowski ${ }^{21}$ and, for any open set $\Omega \subset M$, we first define $F_{C}(\Omega)$ to be the set of smooth 
maps such that $\varphi \mapsto \nabla F_{\varphi}$ is Bastiani smooth from $C^{\infty}(\Omega)$ to $\mathcal{D}(M)$ and, for every integer $k, \varphi \mapsto F_{\varphi}^{(k)}$ is Bastiani smooth from $C^{\infty}(\Omega)$ to $\mathcal{E}_{C_{k}}^{\prime}\left(M^{k}\right)$ [we do not need to index $\mathcal{E}^{\prime}\left(M^{k}\right)$ with two cones because $C_{k}$ is closed ${ }^{21}$. The set $\mathrm{F}_{\text {loc }}(\Omega)$ of local functionals is then the subset of $\mathrm{F}_{C}(\Omega)$ satisfying the addivity condition.

The topology of $F_{C}$ is induced by the family of seminorms given by Eq. (15) that depend on the equicontinuous sets of the dual $\mathcal{D}_{\Lambda_{k}, \bar{\Lambda}_{k}}^{\prime}\left(M^{k}\right)$ of $\mathcal{D}_{C_{k}}^{\prime}\left(M^{k}\right)$, where $\Lambda_{k}=\dot{T}^{*} M^{k} \backslash C_{k}$. They were determined by Dabrowski (Ref. 21, Lemma 28).

Proposition IV.2. A subset $B$ of $\mathcal{D}_{\Lambda_{k}}^{\prime}\left(M^{k}\right)$ is equicontinuous if and only if there is a closed cone $\Gamma \subset \Lambda_{k}$ such that $\operatorname{WF}(u) \subset \Gamma$ for every $u \in B$ and $B$ is bounded in $\mathcal{D}_{\Gamma}^{\prime}\left(M^{k}\right)$.

The bounded sets of $\mathcal{D}_{\Gamma}^{\prime}\left(M^{k}\right)$ are characterized in detail in Ref. 85. The topology of $\mathcal{D}_{\Lambda_{k}}^{\prime}\left(M^{k}\right)$, where $\Lambda_{k}$ is open, can be described as a non-countable inductive limit as follows. Write the complement $\Lambda_{k}^{c}=\cup \Gamma_{n}$, where each $\Gamma_{n}$ is a compactly supported closed set. We write the open set $\Gamma_{n}^{c}$ as a countable union of closed sets $\Gamma_{n}^{c}=\cup_{m} \Lambda_{n, m}$ so that $\Gamma_{n}=\cap_{m} \Lambda_{n, m}^{c}$ and $\Lambda_{k}^{c}=\cup_{n} \cap_{m} \Lambda_{n, m}^{c}$ is a countable union of countable intersections of open sets. We obtain $\Lambda_{k}=\cap_{n} \cup_{m} \Lambda_{n, m}$. We define for a sequence $\alpha$ the closed set $\Pi_{\alpha}=\cap_{n} \Lambda_{n, \alpha(n)}$ such that $\alpha \leq \beta$ implies $\Pi_{\alpha} \subset \Pi_{\beta}$. Then $\Lambda=\cup_{\alpha} \Pi_{\alpha}$ is a non-countable inductive limit of closed cones from which we can define the topology of $\mathcal{D}_{\Lambda_{k}}^{\prime}\left(M^{k}\right)$ as a non-countable inductive limit of $\mathcal{D}_{\Pi_{\alpha}}^{\prime}\left(M^{k}\right)$.

The space $F_{C}$ furnished with the topology induced by the seminorms defined by Eqs. (12) and (13) is complete and nuclear. The space $F_{\text {loc }}$ of local functionals is the closed subset of $F_{C}$ defined by the additivity condition defined in Sec. V. As a closed subspace of a nuclear complete space, the space of local functionals is nuclear and complete.

Further examples of spaces of functionals are given by Dabrowski. ${ }^{22}$

\section{ADDITIVITY}

The characterization of local functionals is a long-standing mathematical problem. According to Rao, ${ }^{88}$ the first criterium was proposed by Pinsker in 1938 and called partial additivity. ${ }^{89}$ This criterium is also used in physics, but we shall see that it is not what we need by exhibiting a partially additive functional which is not local. Then, we shall discuss a more stringent criterium that is exactly what we need.

\section{A. Partial additivity}

When looking for an equation to characterize functionals having the form of Eq. (1), one can make the following observation. Let $\varphi_{1}$ and $\varphi_{2}$ be two smooth functions with disjoint supports $K_{1}$ and $K_{2}$ and assume that $f(x, \varphi(x), \ldots)=0$ if $\varphi=0$ on a neighborhood of $x^{89}$ so that $F(0)=0$. Then, since the support of $\varphi_{1}+\varphi_{2}$ is included in $K_{1} \cup K_{2}$,

$$
\begin{aligned}
F\left(\varphi_{1}+\varphi_{2}\right) & =\int_{K_{1}} d x f\left(x, \varphi_{1}(x)+\varphi_{2}(x), \ldots\right)+\int_{K_{2}} d x f\left(x, \varphi_{1}(x)+\varphi_{2}(x), \ldots\right) \\
& =\int_{K_{1}} d x f\left(x, \varphi_{1}(x), \ldots\right)+\int_{K_{2}} d x f\left(x, \varphi_{2}(x), \ldots\right)=F\left(\varphi_{1}\right)+F\left(\varphi_{2}\right) .
\end{aligned}
$$

Therefore, it is tempting to use the condition of locality,

$$
F\left(\varphi_{1}+\varphi_{2}\right)=F\left(\varphi_{1}\right)+F\left(\varphi_{2}\right),
$$

for $\varphi_{1}$ and $\varphi_{2}$ with disjoint support and functionals $F$ such that $F(0)=0$. And indeed, many authors since 1938, including Gelfand and Vilenkin (Ref. 90, p. 275), used condition (16), but with disjoint support replaced by $\varphi_{1} \varphi_{2}=0$ and smooth functions by measurable functions (see Ref. 88 for a review). In perturbative quantum field theory, partial additivity in our sense is also used when the function $f$ 
in Eq. (1) is polynomial ${ }^{17,91,92}$ because, in that case, partial additivity is equivalent to locality in the sense of Eq. (1). ${ }^{17}$

However, this definition of locality does not suit our purpose, essentially because the set of functions $\varphi$ that can be written as $\varphi=\varphi_{1}+\varphi_{2}$ (with $\left.\operatorname{supp} \varphi \cap \operatorname{supp} \varphi_{2}=\emptyset\right)$ is not dense in the space of smooth functions. We show this now and we construct a partially additive functional which is not local.

\section{B. A non-local partially additive functional}

We work in the space $C^{\infty}\left(\mathbb{S}^{1}\right)$ of smooth functions on the unit circle. We denote by $\mathcal{I}$ the subset of functions $f=\varphi_{1}+\varphi_{2}$ which are sums of the two elements of $C^{\infty}\left(\mathbb{S}^{1}\right)$ whose supports are disjoint. It is not a vector subspace of $C^{\infty}\left(\mathbb{S}^{1}\right)$.

The idea of the construction is the following. In the metric space $C^{\infty}\left(\mathbb{S}^{1}\right)$, we will show that the subset $\mathcal{I}$ is bounded away from the constant function $f=1$. This means that the functional equation (16) only concerns the restriction $\left.F\right|_{\mathcal{I}}$ to a subset which is bounded away from 1 . Therefore there is some open neighborhood of $f=1$ which does not meet $\mathcal{I}$. Then we use Sobolev norms to build some cutoff function $\chi$ to glue a local functional near $\mathcal{I}$ with a nonlocal functional near $f=1$.

Lemma V.1. The constant function $f=1$ is bounded away from $\mathcal{I}$ in $C^{\infty}\left(\mathbb{S}^{1}\right)$ : if $f \in \mathcal{I}$, then $\| f-\left.1\right|_{C^{0}}=\sup _{x \in \mathbb{S}^{1}}|f(x)-1| \geq 1$.

Proof. Let us denote by $\|\cdot\|_{C^{0}}$ the norm $\|f\|_{C^{0}}=\sup _{x \in \mathbb{S}^{1}}|f(x)|$. It is a continuous norm for the Fréchet topology of $C^{\infty}\left(\mathbb{S}^{1}\right)$ because $\|f\|_{C^{0}}=\pi_{0, \mathbb{S}^{1}}(f)$. Then, if supp $\varphi_{1} \cap \operatorname{supp} \varphi_{2}=\emptyset$, we have $\left\|\varphi_{1}+\varphi_{2}-1\right\|_{C^{0}} \geqslant 1$. Indeed, the supports of $\varphi_{1}$ and $\varphi_{2}$ being compact, the fact that they do not meet implies that they are at a finite distance. Thus, there is a point $x \in \mathbb{S}^{1}$ such that $\varphi_{1}(x)=\varphi_{2}(x)=0$. Hence, $\left|\varphi_{1}(x)+\varphi_{2}(x)-1\right|=1$ and $\sup _{x \in \mathbb{S}^{1}}\left|\varphi_{1}(x)+\varphi_{2}(x)-1\right| \geq 1$.

The second step is to build a smooth function $\chi$ such that $\chi(1)=1$ and $\left.\chi\right|_{\mathcal{I}}=0$.

Lemma V.2. There is a smooth function $\chi: C^{\infty}\left(\mathbb{S}^{1}\right) \rightarrow \mathbb{R}$ such that $\chi=1$ on a neighborhood of $f=1$ and $\chi(f)=0$ if $\|f-1\|_{C^{0}} \geq 1$. In particular, $\left.\chi\right|_{\mathcal{I}}=0$.

Proof. First recall that the Sobolev norm $H^{2 k}$ on $\mathbb{S}^{1}$ is defined as

$$
\|f\|_{H^{2 k}}=\sqrt{\int_{\mathbb{S}^{1}}\left((1-\Delta)^{k} f(x)\right)^{2} d x}=2 \pi\left(\left.\sum_{n \in \mathbb{Z}}\left(1+n^{2}\right)^{2 k} \widehat{f}(n)\right|^{2}\right)^{\frac{1}{2}},
$$

where the last representation uses the Fourier series $f(x)=\sum_{n} \hat{f}(n) e^{i n x}$. By the Sobolev injections, $H^{2}\left(\mathbb{S}^{1}\right)$ injects continuously in $C^{0}\left(\mathbb{S}^{1}\right)$. In other words, there is a constant $C>0$ such that $\|f\|_{C^{0}} \leqslant$ $C\|f\|_{H^{2}}$ for every $f \in C^{\infty}\left(\mathbb{S}^{1}\right)$.

Now we take a function $g \in C^{\infty}(\mathbb{R})$ such that $g(t)=1$ when $t \leq 1 / 3 C^{2}$ and $g(t)=0$ when $t \geq$ $1 / 2 C^{2}$ and we define $\chi: \mathbb{C}^{\infty}\left(\mathbb{S}^{1}\right) \rightarrow \mathbb{R}$ by composing $g$ with the square of the Sobolev norm,

$$
\chi(f)=g\left(\|1-f\|_{H^{2}}^{2}\right) .
$$

If $\|1-f\|_{C^{0}} \geqslant 1$ (in particular, if $f \in \mathcal{I}$ by Lemma V.1), the Sobolev injection leads to

$$
1 \leqslant\|1-f\|_{C^{0}} \leqslant C\|1-f\|_{H^{2}} \Longrightarrow\|1-f\|_{H^{2}}^{2} \geqslant \frac{1}{C^{2}}
$$

hence $g\left(\|1-f\|_{H^{2}}^{2}\right)=0$ by definition of $g$. 
On the other hand, $\|1-f\|_{H^{2}} \leqslant \frac{1}{\sqrt{3} C}$ means that $f$ belongs to the neighborhood of the constant function $f=1$ defined by $V=\left\{f ;\|1-f\|_{H^{2}} \leqslant 1 / \sqrt{3} C\right\}$. On this neighborhood, $g\left(\|1-f\|_{H^{2}}^{2}\right)=1$. The smoothness of $\chi$ is an immediate consequence of the chain rule, the smoothness of $g$ and of the squared Sobolev norm $\|\cdot\|_{H^{2}\left(\mathbb{S}^{1}\right)}^{2}$.

We are now ready to define our counterexample:

Theorem V.3. The functional $F_{\mathrm{nl}}$ on $C^{\infty}\left(\mathbb{S}^{1}\right)$ defined for any integer $N>1$ by

$$
F_{\mathrm{nl}}(f)=(1-\chi(f)) \int_{\mathbb{S}^{1}} f(x) d x+\chi(f)\left(\int_{\mathbb{S}^{1}} f(x) d x\right)^{N}
$$

is partially additive but not local.

Proof. For every $\left(\varphi_{1}, \varphi_{2}\right) \in C^{\infty}\left(\mathbb{S}^{1}\right)^{2}$ whose supports are disjoint, $f=\varphi_{1}+\varphi_{2} \in \mathcal{I}$, hence $\chi(f)$ $=0$ by Lemma V.2. Moreover, we saw that if $\operatorname{supp} \varphi_{1} \cap \operatorname{supp} \varphi_{2}=\emptyset$, then there is a point $x \in \mathbb{S}^{1}$ such that $\varphi_{1}(x)=\varphi_{2}(x)=0$. Thus, $\left\|1-\varphi_{1}\right\|_{C^{0}} \geq 1$ and $\left\|1-\varphi_{2}\right\|_{C^{0}} \geq 1$. As a consequence, $\chi\left(\varphi_{1}\right)=\chi\left(\varphi_{2}\right)$ $=0$ by Lemma V.2 and $F_{\mathrm{nl}}\left(\varphi_{1}+\varphi_{2}\right)=\int_{\mathbb{S}^{1}}\left(\varphi_{1}(x)+\varphi_{2}(x)\right) d x=F_{\mathrm{nl}}\left(\varphi_{1}\right)+F_{\mathrm{nl}}\left(\varphi_{2}\right)$.

On the other hand, in the neighborhood $V$ of $f=1$ given by Lemma V.2, $\chi(f)=1$ hence $F_{\mathrm{nl}}(f)=\left(\int_{\mathbb{S}^{1}} f(x) d x\right)^{N}$ which is not local. It is even a typical example of a multilocal functional. ${ }^{16}$

Since partial additivity is equivalent to locality for polynomial functions, the non-locality of $F_{\mathrm{nl}}$ can be considered to be non-perturbative. Moreover, the fact that the derivatives $D^{n} F_{\mathrm{nl}}$ calculated at $f=0$ are supported in the thin diagonal of $\left(\mathbb{S}^{1}\right)^{n}$, although $F_{\mathrm{nl}}$ is not local, means that locality cannot be controlled by the support of differentials taken at a single function $f$. We come now to the property that is relevant for quantum field theory.

\section{Additive functionals}

In 1965, Chacon and Friedman ${ }^{93}$ introduced a more stringent concept of additivity that meets our needs.

Definition V.4. We say that a Bastiani smooth map $F: C^{\infty}(M) \rightarrow \mathbb{K}$ is additive if, for every triple $\left(\varphi_{1}, \varphi_{2}, \varphi_{2}\right)$ of smooth functions on $M$, the property $\operatorname{supp} \varphi_{1} \cap \operatorname{supp} \varphi_{3}=\emptyset$ implies the property

$$
F\left(\varphi_{1}+\varphi_{2}+\varphi_{3}\right)=F\left(\varphi_{1}+\varphi_{2}\right)+F\left(\varphi_{2}+\varphi_{3}\right)-F\left(\varphi_{2}\right) .
$$

In the literature, the additivity equation (19) is also called the Hammerstein property. ${ }^{94-99}$ The additivity property is equivalent to the fact that the functional derivatives are supported on the thin diagonal $D_{n}=\left\{\left(x_{1}, \ldots, x_{n}\right) \in M^{n} ; x_{1}=\cdots=x_{n}\right\} .{ }^{17,92}$

Proposition V.5. A smooth functional $F$ on $C^{\infty}(M)$ is additive if and only if $\operatorname{supp} F_{\varphi}^{(2)} \subset D_{2}$ for every $\varphi \in C^{\infty}(M)$, where $D_{2}=\left\{(x, y) \in M^{2} ; x=y\right\}$. If $F$ is an additive functional, then supp $F_{\varphi}^{(n)} \subset D_{n}$ for every $\varphi \in C^{\infty}(M)$, where $D_{n}=\left\{\left(x_{1}, \ldots, x_{n}\right) \in M^{n} ; x_{1}=\cdots=x_{n}\right\}$.

Proof. We first prove that the second derivative of an additive functional is localized on the diagonal. ${ }^{17}$ If we use the additivity property with $\varphi_{1}=\lambda \psi, \varphi_{3}=\mu \chi$ and $\operatorname{supp} \psi \cap \operatorname{supp} \chi=\emptyset$, then

$$
F\left(\lambda \psi+\varphi_{2}+\mu \chi\right)=F\left(\lambda \psi+\varphi_{2}\right)+F\left(\varphi_{2}+\mu \chi\right)-F\left(\varphi_{2}\right) .
$$

Since no term on the right-hand side of this equation depends on both $\lambda$ and $\mu$, we have

$$
\frac{\partial^{2} F\left(\lambda \psi+\varphi_{2}+\mu \chi\right)}{\partial \lambda \partial \mu}=D^{2} F_{\lambda \psi+\varphi_{2}+\mu \chi}(\psi, \chi)=F_{\lambda \psi+\varphi_{2}+\mu \chi}^{(2)}(\psi \otimes \chi)=0 .
$$


This equation, being true for every $\varphi_{2}$, can be written as $F_{\varphi}^{(2)}(\psi \otimes \chi)=0$ for every $\varphi$ and every pair $(\psi, \chi)$ with disjoint supports. Now for every point $(x, y) \in M^{2}$ such that $x \neq y$, there are two open sets $U_{x}$ containing $x$ and $U_{y}$ containing $y$ such that $U_{x} \cap U_{y}=\emptyset$. Then, any pair of functions $\psi$ and $\chi$ supported in $U_{x}$ and $U_{y}$ satisfies $F_{\varphi}^{(2)}(\psi \otimes \chi)=0$. Since the functions $\psi \otimes \chi$ are dense in $\mathcal{D}\left(M^{2}\right)$, this implies that every test functions $f \in \mathcal{D}\left(M^{2}\right)$ supported in $U_{x} \times U_{y}$ satisfies $F_{\varphi}^{(2)}(f)=0$. Thus $(x, y) \notin \operatorname{supp} F_{\varphi}^{(2)}$ and $\operatorname{supp} F_{\varphi}^{(2)} \subset D_{2}$. To determine the support of $F_{\varphi}^{(n)}$, consider a point $\left(x_{1}, \ldots\right.$, $x_{n}$ ) which is not in $D_{n}$. Then, there are two indices $i$ and $j$ such that $x_{i} \neq x_{j}$. Denote by $U_{x}$ an open neighborhood of $x_{i}$ and by $U_{y}$ an open neighborhood of $x_{j}$ and repeat the previous proof to obtain $F_{\varphi}^{(2)}(\psi \otimes \chi)=0$ for every $\varphi$ and every pair $(\psi, \chi)$ with supports in $U_{x}$ and $U_{y}$. Now, rewrite $\varphi=\varphi_{0}$ $+\sum \lambda_{k} \psi_{k}$, where $\psi_{k}\left(x_{k}\right) \neq 0$ and the sum is over all integers from 1 to $n$ except $i$ and $j$. Then, the derivatives with respect to $\lambda_{k}$ are all zero and we find again with the same argument that $\left(x_{1}, \ldots, x_{n}\right)$ is not in the support of $F_{\varphi}^{(n)}$ for every $\varphi$.

Conversely, ${ }^{20,92}$ assume that supp $F_{\varphi}^{(2)} \subset D_{2}$ for every $\varphi$. As we have seen in the first part of the proof, this means that if $\psi$ and $\chi$ have disjoint support, then $D^{2} F_{\varphi}(\psi, \chi)=F_{\varphi}^{(2)}(\psi \otimes \chi)=0$. By the fundamental theorem of calculus,

$$
\begin{gathered}
F(\varphi+\psi+\chi)=F(\varphi+\psi)+\int_{0}^{1} d \mu \frac{d}{d \mu} F(\varphi+\psi+\mu \chi), \\
F(\varphi+\psi+\mu \chi)=F(\varphi+\mu \chi)+\int_{0}^{1} d \lambda \frac{d}{d \lambda} F(\varphi+\lambda \psi+\mu \chi) .
\end{gathered}
$$

Thus,

$$
F(\varphi+\psi+\chi)=F(\varphi+\psi)+\int_{0}^{1} d \mu \frac{d}{d \mu} F(\varphi+\mu \chi)+\int_{0}^{1} d \lambda \int_{0}^{1} d \mu \frac{\partial^{2}}{\partial \lambda \partial \mu} F(\varphi+\lambda \psi+\mu \chi) .
$$

The last term is zero because $D^{2} F_{\varphi}(\psi, \chi)=0$ and the second term is $F(\varphi+\chi)-F(\varphi)$. We recover the additivity condition.

Finally, additivity is stronger than partial additivity because the latter corresponds to the case $\varphi_{2}$ $=0$ and $F(0)=0$. It is strictly stronger because $F_{\mathrm{nl}}$ is not additive.

\section{CHARACTERIZATION OF SMOOTH LOCAL FUNCTIONALS}

In this section, we give a characterization of local functionals inspired by the topology described in Sec. IV E. In the sequel, we shall deal with compactly supported distributions $u$ with empty wavefront sets. We repeat the definition of local functionals in terms of jets.

Definition VI.1. Let $U$ be an open subset of $C^{\infty}(M)$. A Bastiani smooth functional $F: U \rightarrow \mathbb{K}$ is said to be local if, for every $\varphi \in U$, there is a neighborhood $V$ of $\varphi$, an integer $k$, an open subset $\mathcal{V} \subset J^{k} M$, and a smooth function $f \in C^{\infty}(\mathcal{V})$ such that $x \in M \mapsto f\left(j_{x}^{k} \psi\right)$ is supported in a compact subset $K \subset M$ and

$$
F(\varphi+\psi)=F(\varphi)+\int_{M} f\left(j_{x}^{k} \psi\right) d x
$$

whenever $\varphi+\psi \in V$ and where $j_{x}^{k} \psi$ denotes the $k$-jet of $\psi$ at $x$.

We invite the reader not familiarized with jet bundles to have a look at Sec. VI A, where these objects are carefully defined. Note that the representation of $F$ by $f$ is not unique: adding the total derivative of a function does not change the result. We shall see that $f$ belongs to a unique cohomology class for some specific cohomology theory on the space of local functionals. 
Before we state the main theorem of this section, let us start by some useful definition-lemma:

Lemma VI.2. Let $U$ be an open subset of $C^{\infty}(M)$ and $F: U \rightarrow \mathbb{K}$ be Bastiani smooth. For every $\varphi$ such that the distribution $D F_{\varphi} \in \mathcal{E}^{\prime}(M)$ has an empty wave front set, there exists a unique function $\nabla F_{\varphi} \in \mathcal{D}(M)$ such that

$$
D F_{\varphi}[h]=\int_{M} \nabla F_{\varphi}(x) h(x) d x
$$

Proof. Once a density $d x$ is fixed on $M$, functions in $L_{l o c}^{1}(M)$ [in particular in $C^{\infty}(M)$ ] can be identified with distributions by the map

$$
f \in L_{l o c}^{1}(M) \mapsto\left(\phi \mapsto \int_{M} f \phi d x\right)
$$

and Ref. 32, Theorem 1.2.4, shows that the distribution is uniquely defined when $f$ is continuous hence when $f$ is smooth.

Since $W F\left(D F_{\varphi}\right)=\emptyset$, there exists a unique $C^{\infty}$ function $\nabla F_{\varphi}$ which represents the distribution $D F_{\varphi} \in \mathcal{E}^{\prime}(M)$ by integration on $M$ against $d x$.

The main theorem of this section is

Theorem VI.3. Let $U$ be an open subset of $C^{\infty}(M)$ and $F: U \rightarrow \mathbb{K}$ be Bastiani smooth. Then, $F$ is local if and only if the following two conditions are satisfied:

1. $F$ is additive.

2. For every $\varphi \in U$, the differential $D F_{\varphi}=F_{\varphi}^{(1)}$ of $F$ at $\varphi$ has an empty wave front set and the map $\varphi \mapsto \nabla F_{\varphi}$ is Bastiani smooth from $U$ to $\mathcal{D}(M)$.

Note that our definition of locality is strictly more general than the usual one because the counterexample described in Lemma III.12 is local in our sense but not in the sense of Eq. (1) since its order is infinite.

The proof is delayed to Sec. VI C. Since this theorem deals with jets, we start with a short presentation of the jet bundle. Our point of view on jets is based on the concept of infinitesimal neighborhoods due to Grothendieck and is closely related to several expositions in the literature. ${ }^{100-102}$

\section{A. The manifold of jets of functions on a manifold}

Let $M$ be a manifold. For every smooth real-valued function $\varphi$ on $M$, we call $k$-jet of $\varphi$ at a point $x \in M$ the class $j_{x}^{k}(\varphi)$ of $\varphi$ in the quotient $C^{\infty}(M) / I_{x}^{k+1}$, with the understanding that $I_{x}^{k+1}$ stands for the $(k+1)$-th power of the ideal $I_{x}$ of smooth functions on $M$ vanishing at $x \in M$. Recall that $I_{x}^{k+1}$ coincides with the ideal of smooth functions on $M$ whose $k+1$ first derivatives vanish at the point $x$.

For all $x \in M$, the space $J_{x}^{k}(M)$ of all $k$-jets of functions on $M$ at $x$ coincides with $C^{\infty}(M) / I_{x}^{k+1}$ and is called the space of $k$-jets at $x$. It is clearly a vector space. The disjoint union $J^{k}(M):=\bigsqcup_{x \in M} J_{x}^{k}(M)$ is a smooth vector bundle over $M$ called the bundle of $k$ th jets. Consider the map

$$
\begin{aligned}
\mathfrak{J}_{\Delta}: C^{\infty}(M \times M) & \rightarrow \Gamma\left(J^{k}(M)\right) \\
\psi & \mapsto x \mapsto j_{x}^{k}\left(\mathfrak{i}_{x}^{*} \psi\right),
\end{aligned}
$$

where $\mathfrak{i}_{x}: M \rightarrow M \times M$ is the map $y \mapsto(x, y)$. It is known that $\mathfrak{I}_{\Delta}$ is surjective onto the space of smooth sections of $J^{k}(M)$ and its kernel is the $(k+1)$-th power of the ideal $\mathcal{I}_{\Delta}$ of functions on $M \times$ $M$ vanishing on the diagonal.

Last, the projection $p_{1}: M \times M \rightarrow M$ onto the first component dualizes in an algebra morphism $\varphi \mapsto p_{1}^{*} \varphi$ from $C^{\infty}(M)$ to $C^{\infty}(M \times M)$ which endows $C^{\infty}(M \times M)$ with a $C^{\infty}(M)$-module structure. The space of sections of $J^{k}(M)$ is also a $C^{\infty}(M)$-module, and it is routine to check that $\mathfrak{J}_{\Delta}$ is a morphism of $C^{\infty}(M)$-modules. Therefore, the space of sections of $J^{k}(M)$ is, as a $C^{\infty}(M)$-module, 
isomorphic to the quotient $C^{\infty}(M \times M) / \mathcal{I}_{\Delta}^{k+1}$ and $\Gamma\left(J^{k}(M)\right)$ fits into the following exact sequence of $C^{\infty}(M)$-modules:

$$
0 \rightarrow \mathcal{I}_{\Delta}^{k+1} \rightarrow C^{\infty}(M \times M) \rightarrow \Gamma\left(J^{k}(M)\right) \simeq C^{\infty}(M \times M) / \mathcal{I}_{\Delta}^{k+1} \rightarrow 0 .
$$

And the map that to $f$ associates its $k$-jet reads

$$
f \in C^{\infty}(M) \mapsto\left[\left(p_{1}^{*} f\right)\right] \in C^{\infty}(M \times M) / \mathcal{I}_{\Delta}^{k+1} .
$$

The purpose of the rest of this section is to prove the technical proposition VI.4, the statement of which we now explain. For all integers $k \in \mathbb{N}$, there is a natural vector bundle morphism $\mathfrak{J}^{k}$ from the trivial bundle over $M$ with typical fiber $E=C^{\infty}(M)$ to the bundle $J^{k}(M) \rightarrow M$ of $k$-jets. This morphism simply consists in assigning to a pair $(f, x)$ in $E \times M \rightarrow M$ the $k$-jet of $f$ at $x$. In equation,

$$
\begin{aligned}
\mathfrak{J}^{k}: E \times M & \mapsto j^{k}(M), \\
(f, x) & \rightarrow j_{x}^{k}(f) .
\end{aligned}
$$

The result goes as follows.

Proposition VI.4. Let $E=C^{\infty}(M), V \subset E$ be an open subset, and $k \in \mathbb{N}$ be an integer.

1. The subset $\mathfrak{J}^{k}(V \times M)$ is an open subset of $j^{k}(M)$.

2. Let $c$ be a smooth $\mathbb{K}$-valued function on $V \times M$, with $V \subset E$ being an open subset. Assume that $c(f, x)$ depends only on the $k$-jet of the function fat the point $x$. Then there exists a unique smooth $\mathbb{K}$-valued function $\tilde{c}$ on the open subset $\mathfrak{J}^{k}(V \times M) \subset j^{k}(M)$ that makes the following diagram commutative:

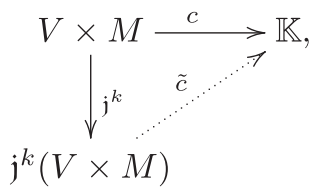

i.e., such that the relation $c(f, x)=\tilde{c}\left(j_{x}^{k}(f)\right)$ holds for all $f \in V$ and $x \in M$.

When $V=E$, Proposition VI.4 specializes to the following easier statement:

Corollary VI.5. Let c be a smooth function from $E \times M$ to $\mathbb{K}$. Assume that there exists an integer $k$ such that $c(f, x)$ depends only on the $k$-jet of the function $f$ at the point $x$. Then there exists a unique smooth $\mathbb{K}$-valued function $\tilde{c}$ on $j^{k}(M)$ such that the following diagram commutes:

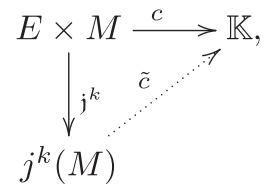

i.e., such that the relation $c(f, x)=\tilde{c}\left(j_{x}^{k}(f)\right)$ holds for all $f \in E$ and $x \in M$.

Before establishing these results, we shall need several lemmas.

Lemma VI.6. The vector bundle morphism $\mathfrak{J}^{k}$ described in (21) is surjective and admits a smooth section $\mathfrak{s}^{k}$.

Proof. The section $\mathfrak{s}^{k}$, when it exists, is by construction a right inverse of $\mathfrak{J}^{k}$, the latter is surjective. It suffices therefore to prove the existence of $s^{k}$.

We first prove that the lemma holds true for $M$ an open subset $V$ of $\mathbb{R}^{d}$. In that case, the bundle of $k$-jets $j_{x}^{k}(V)$ is isomorphic to the trivial bundle over $V$ with a typical fiber, the space of polynomials of degree less or equal to $k$. There is an obvious candidate for the section of $\mathfrak{J}^{k}$ : it consists in mapping $\alpha_{x} \in j_{x}^{k}(V)$ to the unique polynomial of degree $k$ whose $k$-jet at $x \in V$ is $\alpha$. The henceforth obtained 
assignment, which we denote by $\mathfrak{s}_{V}^{k}$, is a smooth vector bundle morphism from $j^{k}(V) \rightarrow V$ to the trivial bundle $C^{\infty}(V) \times V \rightarrow V$. It is by construction a section of $\mathfrak{J}^{k}$.

We now go back to the general case of an arbitrary manifold $M$. For every point $x \in M$, choose $V_{x}$ a coordinate neighborhood and let $\chi$ be a smooth function with compact support on $V_{x}$ which is identically equal to $1 \mathrm{in}$ a neighborhood $V_{x}^{\prime} \subset V_{x}$ of $x$. Since $V_{x}$ is a coordinate neighborhood, it can be identified with an open subset of $\mathbb{R}^{d}$, which allows us to consider

$$
\mathfrak{s}_{V_{x}}^{k}: j^{k}\left(V_{x}\right) \mapsto C^{\infty}\left(V_{x}\right) \times V_{x}
$$

as in the previous paragraph. We can then consider the composition of vector bundle morphisms over $V_{x}$,

$$
\left.j^{k}(M)\right|_{V_{x}} \simeq j^{k}\left(V_{x}\right) \stackrel{\mathfrak{s}_{V_{x}}^{k}}{\longrightarrow} C^{\infty}\left(V_{x}\right) \times V_{x} \stackrel{m_{x} \times i d}{\longrightarrow} E \times V_{x},
$$

where $\left.j^{k}(M)\right|_{V_{x}} \simeq j^{k}\left(V_{x}\right)$ is the obvious identification of the $k$-jet bundle of $V_{x}$ to the restriction to $V_{x}$ of the $k$-jet bundle on $M$ and where $m_{\chi}$ is the smooth linear map from $C^{\infty}\left(V_{x}\right)$ to $E=C^{\infty}(M)$ defined by $m_{\chi}(f)=f \chi$. Since $\chi$ is identically equal to 1 on $V_{x}^{\prime}$, the restriction to $V_{x}^{\prime}$ of this vector bundle morphism is by construction a section of the restriction of $\mathfrak{J}^{k}$ to $V_{x}^{\prime}$.

Since the manifold $M$ is paracompact, ${ }^{103}$ the latter point implies that the manifold $M$ can be covered by open subsets $\left(U_{i}\right)_{i \in I}$ such that the restriction of $\mathfrak{J}^{k}$ to $U_{i}$ admits a section $\mathfrak{s}_{i}^{k}$. Without any loss of generality, we can assume the existence of a smooth partition of unity $\left(\chi_{i}\right)_{i \in I}$ relative to this open cover. A global smooth section of $\mathfrak{J}^{k}$ is then given by the explicit formula $\mathfrak{s}^{k}=\sum_{i \in I} \chi_{i} \mathfrak{s}_{i}^{k}$, as follows from the obvious computation:

$$
\mathfrak{J}^{k} \circ \mathfrak{s}^{k}=\sum_{i \in I} \chi_{i} \mathfrak{J}^{k} \circ \mathfrak{s}_{i}^{k}=\sum_{i \in I} \chi_{i} \operatorname{id}_{j^{k}(M)}=\operatorname{id}_{j^{k}(M)},
$$

where we used the fact that $\mathrm{j}^{k}$ commutes with multiplications by $\chi_{i}$ since $\mathrm{j}^{k}: E \times M \mapsto J^{k} M$ is a vector bundle morphism. This completes the proof.

Since $c: V \times M \rightarrow \mathbb{K}$ is only defined on the open subset $V \times M$ of $E \times M$, we need the following refinement of Lemma VI.6 where the local sections $t_{x}^{k}$ of $\mathfrak{J}^{k}$ are valued in $V \times M$ :

Lemma VI.7. For every $(f, x) \in E \times M$, the vector bundle morphism $\mathfrak{J}^{k}$ described in (21) admits a smooth section $\mathrm{t}^{k}$ through ${ }^{104}(f, x)$.

Proof. Notice that Lemma VI.7 can be derived from Lemma VI.6 for any vector bundle morphism over the identity of $M$. A careful check shows that the arguments below are absolutely general and indeed show that for any two vector bundles $E_{1}, E_{2}$ over $M$, any vector bundle morphism $E_{1} \mapsto E_{2}$ over $M$ that admits a section is a submersion and admits a section through every point of $E_{1}$.

We prefer to do it, however, in our particular setting-since one of the bundles is infinite dimensional and requires careful attention.

Let $\mathfrak{s}^{k}$ be a section of $\mathfrak{J}^{k}$ as in Lemma VI.6. Consider the smooth map defined at all points $y \in$ $M$ by

$$
\begin{aligned}
\mathfrak{t}_{y}^{k}: j_{y}^{k}(M) & \rightarrow(E \times M)_{y} \simeq E, \\
\beta & \mapsto \mathfrak{s}_{y}^{k}(\beta)+\left(f-\mathfrak{s}_{y}^{k} \circ j_{y}^{k}(f)\right) .
\end{aligned}
$$

This map is smooth by construction. It is again a section $\mathfrak{J}^{k}$, as follows from the following computation, valid for all $y \in M, \beta \in j_{y}^{k}(M)$ :

$$
\begin{aligned}
\mathfrak{J}_{y}^{k} \circ \mathfrak{t}_{y}^{k}(\beta) & =\mathfrak{J}_{y}^{k}\left(\mathfrak{s}_{y}^{k}(\beta)+\left(f-\mathfrak{s}_{y}^{k} \circ j_{y}^{k}(f)\right)\right) \\
& =\mathfrak{J}_{y}^{k} \circ \mathfrak{s}_{y}^{k}(\beta)+\mathfrak{J}_{y}^{k}(f)-\mathfrak{J}_{y}^{k} \circ \mathfrak{s}_{y}^{k} \circ j_{y}^{k}(f) \\
& =\beta+\mathfrak{J}_{y}^{k}(f)-\mathfrak{J}_{y}^{k}(f)=\beta .
\end{aligned}
$$


Then the section $\mathrm{t}^{k}$ above satisfies by construction

$$
\mathrm{t}_{x}^{k} \circ \mathfrak{J}_{x}^{k}(f)=\mathfrak{s}_{x}^{k} \circ j_{x}^{k}(f)+f-\mathfrak{s}_{x}^{k} \circ j_{x}^{k}(f)=f .
$$

This completes the proof.

Lemma VI.7 has the following immediate consequence.

Lemma VI.8. The vector bundle morphism $\mathfrak{J}^{k}$ described in (21) is a submersion.

Proof. For every $(f, x)$ in $E \times M$, let us choose $\mathrm{t}^{k}$ to be a section through $(f, x)$ as in Lemma VI.7. By construction, the differential of $\mathfrak{J}^{k}$ at $(f, x)$ admits the differential of $\mathrm{t}^{k}$ at $\mathfrak{J}^{k}(f, x)$ as right inverse, so it is surjective.

We can now prove Proposition VI.4.

Proof. Since the vector bundle morphism $\mathfrak{J}^{k}$ described in (21) is a submersion by Lemma VI.8 and since $V \times M$ is open in $E \times M$, the subset $\mathfrak{J}^{k}(V \times M)$ is an open subset of $j^{k}(M)$. This proves the first item in Proposition VI.4.

Let us now prove the second item. Assume that we are given a function $c: V \times M \mapsto \mathbb{K}$ such that the value $c(f, x)$ at an arbitrary $f \in E$ and $x \in M$ depends only the $k$-jet of $f$ at $x$. The existence of an unique function $\tilde{c}$ from $j^{k}(M)$ to $\mathbb{K}$ making the diagram (22) commute is simply a set-theoretic property: the difficulty is to show that this function $\tilde{c}$ is smooth.

When $V=E$ (i.e., under the assumptions of Corollary VI.5), the smoothness of $\tilde{c}$ follows directly from Lemma VI.6, which implies that the commutative diagram (23) can be completed to

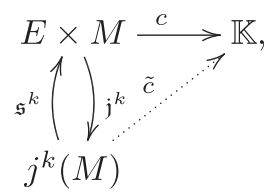

which amounts to say that the following relation holds:

$$
\tilde{c}=c \circ \mathfrak{s}^{k} .
$$

The latter formula and the smoothness of $\mathfrak{s}^{k}$ implies that when $c$ is assumed to be a smooth function, so is the function $\tilde{c}$ by composition. This proves Corollary VI.5.

For the general case, we have to choose, for all $\alpha \in \mathfrak{J}^{k}(V \times M)$, a section $\mathrm{t}^{k}$ of $\mathfrak{J}^{k}$ such that $\mathrm{t}^{k}(\alpha) \in V \times M$. Such a section $\mathrm{t}^{k}$ always exists by Lemma VI.7. Since $\mathrm{t}^{k}$ is smooth, there exists a neighborhood $W_{\alpha}$ of $\alpha$ in $\mathfrak{J}^{k}(V \times M)$ on which $\mathrm{t}^{k}$ takes values in the domain of definition $V \times M$ of $c$, which implies that the commutative diagram (22) can be completed to

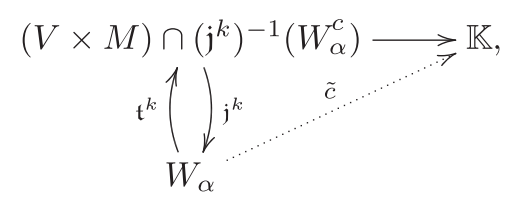

In turn, the commutativity of this diagram gives the explicit description of $\tilde{c}$ through the following formula, valid on $W_{\alpha}$ :

$$
\tilde{c}=c \circ \mathrm{t}^{k} .
$$

Formula (25) and the smoothness of $\mathrm{t}^{k}$ imply that when $c$ is assumed to be a smooth function, so is, by composition, the restriction to $W_{\alpha}$ of the function $\tilde{c}$. Since every $\alpha \in \mathfrak{J}^{k}(V \times M)$ admits a neighborhood on which the restriction of $\tilde{c}$ is smooth, the function $\tilde{c}$ is a smooth function. This completes the proof. 


\section{B. Properties of $\boldsymbol{F}_{\varphi}^{(2)}$}

We first show that the two assumptions of our theorem are equivalent to some strong assumptions on the second derivative of $F$ :

Lemma VI.9. Let $U$ be an open subset of $C^{\infty}(M)$ and $F: U \rightarrow \mathbb{K}$ be Bastiani smooth. Assume that for every $\varphi \in U$, the differential $D F_{\varphi}=F_{\varphi}^{(1)}$ of $F$ at $\varphi$ has no wave front set, i.e., $\operatorname{WF}\left(F_{\varphi}^{(1)}\right)=\emptyset$. Then the two following properties are equivalent:

1. $F$ is additive and the map $\varphi \mapsto \nabla F_{\varphi}$ is Bastiani smooth from $U$ to $\mathcal{D}(M)$.

2. For every $\varphi_{0} \in U$, there is a neighborhood $V$ of $\varphi_{0}$, a compact $K \subset M$, and a finite family of Bastiani smooth maps $f_{\alpha}: V \rightarrow \mathcal{D}(K)$ with $|\alpha| \leqslant k$ such that in any system of local coordinates $(x, y)$ on $M^{2}$,

$$
F_{\varphi}^{(2)}(x, y)=\sum_{|\alpha| \leqslant k} f_{\alpha}(\varphi)(x) \partial_{y}^{\alpha} \delta(x-y)
$$

for every $\varphi \in V$.

In particular, both conditions imply that $D^{2} F_{\varphi}$ is represented by a distribution $F_{\varphi}^{(2)}$ whose wave front set is the conormal bundle of the diagonal in $M^{2}$ (Ref. 105, p. 32).

In the sequel, we shall often use the following simple lemma:

Lemma VI.10. Let $E, F$, and $G$ be locally convex spaces. If $f: E \rightarrow F$ is Bastiani smooth and $\ell$ : $F \rightarrow G$ is linear and continuous, then $\ell \circ f: E \rightarrow G$ is Bastiani smooth and $D^{k}(\ell \circ f)=\ell \circ D^{k} f$.

Proof. This is a consequence of three facts: the map $\ell$ is Bastiani smooth because it is linear and continuous, $\ell \circ f$ is Bastiani smooth because it is the composition of two Bastiani smooth maps, and the chain rule.

We also need the following lemma in the proof of Lemma VI.9:

Lemma VI.11. Let $U$ be a convex open subset of $E=C^{\infty}(M)$ containing the origin and $F: U \rightarrow$ $E$ be a Bastiani smooth map. Then, $G$ : $U \rightarrow E$ defined by $G(\varphi)=\int_{0}^{1} F(s \varphi) d s$ is Bastiani smooth.

Proof. The first step is to define a candidate for the Bastiani differential $D^{k} G$ by determining $D^{k} G(x)$ pointwise in $x \in M$. For every $\left(t_{1}, \ldots, t_{k}, x\right) \in[0,1]^{k} \times M$ and $\left(\varphi, \psi_{1}, \ldots, \psi_{k}\right) \in U \times E^{k}$, the function $\left(t_{1}, \ldots, t_{k}, x\right) \mapsto \int_{0}^{1} d s F\left(s\left(\varphi+t_{1} \psi_{1}+\cdots+t_{k} \psi_{k}\right)\right)(x)$ is smooth in $\left(t_{1}, \ldots, t_{k}, x\right)$ by dominated convergence theorem since $x$ can always be restricted to some compact subset $K \subset M$ to obtain uniform bounds. We can differentiate in $\left(t_{1}, \ldots, t_{k}\right)$ outside and inside the integral and both differentials coincide. Therefore, for every $x \in M$, the Bastiani $k$ th-differential $D^{k} G(x)$ of $G(x)=\int_{0}^{1} d s F(s \varphi(x))$ exists and satisfies the relation $D^{k} G_{\varphi}\left(\psi_{1}, \ldots, \psi_{k}\right)(x)=\int_{0}^{1} d s s^{k} D^{k} F_{s \varphi}\left(\psi_{1}, \ldots, \psi_{k}\right)(x)$. Let us show that $D^{k} G: U \times E^{k} \mapsto E$ is jointly continuous in $\left(\varphi, \psi_{1}, \ldots, \psi_{k}\right)$.

We know that the map $\chi:\left(s, \varphi, \psi_{1}, \ldots, \psi_{k}\right) \in[0,1] \times U \times E^{k} \mapsto s^{k} D^{k} F_{s \varphi}\left(\psi_{1}, \ldots, \psi_{k}\right) \in E$ is continuous by joint continuity of $D^{k} F: U \times E^{k} \mapsto E$ and composition of the continuous maps

$$
(s, \varphi, \psi) \mapsto(s \varphi, \psi) \mapsto s^{k} D^{k} F_{s \varphi}(\psi),
$$

where $\psi=\left(\psi_{1}, \ldots, \psi_{k}\right)$. Then by Ref. 40, Theorem 2.1.5, p. 72, applied to the function $\chi$, the integrated map $\left(\varphi, \psi_{1}, \ldots, \psi_{k}\right) \mapsto \int_{0}^{1} d s s^{k} D^{k} F_{s \varphi}\left(\psi_{1}, \ldots, \psi_{k}\right)$ is continuous and the proof is complete because continuity holds true for every $k$.

Let us now prove Lemma VI.9.

Proof. First of all, by Proposition V.5, $F$ is additive if and only if its second derivative is represented by a distribution supported in the diagonal. We start by proving the direct sense assuming that $\varphi \in U \mapsto \nabla F_{\varphi} \in C^{\infty}(M)$ is Bastiani smooth. 
We first show that item 1 implies item 2 in Lemma VI.9. Since $F$ is Bastiani smooth for any $\varphi_{0} \in$ $U$, we already know by Proposition III.4 that there is some neighborhood $V$ of $\varphi_{0}$ on which $\left.F\right|_{V}$ has fixed compact support that we denote by $K$. Therefore, $\nabla F_{\varphi}$ belongs to $\mathcal{D}(K)$ for every $\varphi \in V$ and $F_{\varphi}^{(2)}$ is supported in $K \times K$. Since $F_{\varphi}^{(2)}$ is also supported in the diagonal of $M^{2}$ by Proposition V.5, the support of $F_{\varphi}^{(2)}$ is contained in the diagonal of $K \times K$ which can be identified with $K$ itself.

Since $D F_{\varphi}$ has an empty wavefront set by assumption, its singular support is empty and it can be represented by a unique smooth compactly supported function $\nabla F_{\varphi}$ (Ref. 32, p. 37) such that

$$
\left.\frac{d}{d t} F(\varphi+t h)\right|_{t=0}=D F_{\varphi}(h)=\int_{M} \nabla F_{\varphi}(x) h(x) d x .
$$

The main step is to represent $F_{\varphi}^{(2)}$ as the Bastiani differential of $\nabla F_{\varphi}$ by calculating the second derivatives in two different ways. The Bastiani smoothness of $F$ yields

$$
\begin{aligned}
D^{2} F_{\varphi}(g, h) & =\left.\frac{d^{2}}{d t_{1} d t_{2}} F\left(\varphi+t_{1} h+t_{2} g\right)\right|_{t_{1}=t_{2}=0} \\
& =\left.\frac{d}{d t_{2}}\left(\left.\frac{d}{d t_{1}} F\left(\varphi+t_{1} h+t_{2} g\right)\right|_{t_{1}=0}\right)\right|_{t_{2}=0} \\
& =\left.\frac{d}{d t_{2}}\left(\int_{M} \nabla F_{\varphi+t_{2} g}(x) h(x) d x\right)\right|_{t_{2}=0},
\end{aligned}
$$

where we used the Schwarz lemma and Eq. (27). To justify switching $\frac{d}{d t_{2}}$ and integration over $M$, observe that the map $\varphi \in U \mapsto \nabla F_{\varphi} \in \mathcal{D}(M)$ is Bastiani smooth, hence $C^{1}$. It follows by the chain rule that $t \mapsto \frac{d}{d t} \nabla F_{\varphi+t g}$ is a $C^{0}$ map valued in $\mathcal{D}(M)$. Since $\nabla F_{\varphi}$ is actually in $\mathcal{D}(K)$ for every $\varphi \in V$ and the topology induced by $\mathcal{D}(M)$ on $\mathcal{D}(K)$ is the usual Fréchet topology of $\mathcal{D}(K)$, the map $\nabla F$ is smooth from $V$ to the Fréchet space $\mathcal{D}(K)$.

Since $\mathcal{D}(K)$ injects continuously in $\left(C^{0}(K), \pi_{0, K}\right)$, this implies that $(t, x) \in[-1,1] \times K \mapsto$ $\frac{d}{d t} \nabla F_{\varphi+t g}(x) \in C^{0}([-1,1] \times K)$. Hence the integrand $\frac{d}{d t_{2}} \nabla F_{\varphi+t_{2} g}(x) h(x)$ is in $C^{0}([-1,1] \times K)$ and is bounded on the integration domain. A continuous map $u: t \in[-1,1] \mapsto u(t,.) \in\left(C^{0}(K), \pi_{0, K}\right)$ corresponds to a map also denoted by $u \in C^{0}([-1,1] \times K)$. Indeed for every convergent sequence $\left(t_{n}, x_{n}\right) \underset{n \rightarrow \infty}{\rightarrow}(t, x)$ in $[-1,1] \times K$, the simple estimate $\left|u(t, x)-u\left(t_{n}, x_{n}\right)\right| \leqslant\left|u(t, x)-u\left(t, x_{n}\right)\right|+\mid u\left(t, x_{n}\right)-$ $u\left(t_{n}, x_{n}\right)|\leqslant| u(t, x)-u\left(t, x_{n}\right) \mid+\pi_{0, K}\left(u\left(t_{n},.\right)-u(t,).\right)$ shows that $u\left(t_{n}, x_{n}\right) \underset{n \rightarrow \infty}{\rightarrow} u(t, x)$.

By the dominated convergence theorem, we can differentiate under the integral sign,

$$
D^{2} F_{\varphi}(g, h)=\left.\frac{d}{d t_{2}}\left(\int_{M} \nabla F_{\varphi+t_{2} g}(x) h(x) d x\right)\right|_{t_{2}=0}=\int_{M}\left(\left.\frac{d}{d t_{2}} \nabla F_{\varphi+t_{2} g}(x)\right|_{t_{2}=0}\right) h(x) d x .
$$

By definition $\left.\frac{d}{d t_{2}} \nabla F_{\varphi+t_{2} g}\right|_{t_{2}=0}$ is only the Bastiani derivative

$$
D \nabla F:(\varphi, g) \in V \times C^{\infty}(M) \mapsto D \nabla F_{\varphi}[g] \in \mathcal{D}(K),
$$

where $D \nabla F$ is a Bastiani smooth map since $\nabla F$ is Bastiani smooth.

Note also that by Theorem III.9, the second derivative $D^{2} F_{\varphi}(g, h)$ can be represented by a map $\varphi \in U \mapsto F_{\varphi}^{(2)} \in \mathcal{E}^{\prime}\left(M^{2}\right)$ such that $\forall(\varphi, g, h) \in U \times C^{\infty}(M)^{2}, D^{2} F_{\varphi}(g, h)=\left\langle F_{\varphi}^{(2)}, g \otimes h\right\rangle$, which means that $F_{\varphi}^{(2)}$ is the distributional kernel of the second derivative $D^{2} F_{\varphi}$. We now arrive at the following equality that identifies two different representations of the second derivative. For every $(\varphi, g, h) \in U$ $\times C^{\infty}(M)^{2}$,

$$
\left\langle F_{\varphi}^{(2)}, g \otimes h\right\rangle=\int_{M} D \nabla F_{\varphi}[g](y) h(y) d y .
$$

By the same theorem III.9 and the chain rule, $D \nabla F_{\varphi}[g](y)=e v_{y} D \nabla F_{\varphi}[g]$ is linear continuous in $g$ $\in C^{\infty}(M)$, hence there is a distribution, denoted by $D \nabla F_{\varphi}(x, y)$, such that $\int_{M} D \nabla F_{\varphi}(x, y) g(x) d x=$ 
$D \nabla F_{\varphi}[g](y)$ and $\int_{M} D \nabla F_{\varphi}(x, y) g(x) d x$ is in $\mathcal{D}(K)$ by Eq. (28). Since the above identity holds for all $(g, h) \in C^{\infty}(M)^{2}$, we have in the sense of distributions that $F_{\varphi}^{(2)}(x, y)=D \nabla F_{\varphi}(x, y)$ where the map

$$
(\varphi, g) \in V \times C^{\infty}(M) \mapsto \int_{M} F_{\varphi}^{(2)}(x, \cdot) g(x) d x \in \mathcal{D}(K)
$$

is Bastiani smooth.

It suffices to do the last part of the proof, which is local in nature, on $M=\mathbb{R}^{d}$. We now represent $F_{\varphi}^{(2)}(x, y)$ as a $C^{\infty}(M)$-linear combination of derivatives of Dirac distributions concentrated on the diagonal. By Proposition V.5, the additive property satisfied by $F$ implies that the distribution $F^{(2)}$ associated with the second derivative $D^{2} F$ is supported in the diagonal $D_{2} \subset M \times M$. By Proposition III.11, the kernel $F_{\varphi}^{(2)}(x, y) \in \mathcal{E}^{\prime}(M \times M)$ has bounded distributional order uniformly in $\varphi \in$ $V$. Schwartz' theorem on distributions supported on a submanifold (Ref. 27, p. 101) states that in local coordinates, there exists a finite sequence of distributions $\left(\varphi \in V \mapsto f_{\alpha}(\varphi, .) \in \mathcal{D}(K)\right)_{|\alpha| \leqslant k}$ such that $F_{\varphi}^{(2)}=\sum_{|\alpha| \leqslant k} f_{\alpha}(\varphi, x) \partial_{y}^{\alpha} \delta(x-y)$. We denote the distributions $f_{\alpha}(\varphi)$ by $f_{\alpha}(\varphi, x)$ because we shall show that $\varphi \mapsto f_{\alpha}(\varphi)$ is Bastiani smooth from $V$ to $\mathcal{D}(K)$.

By Eq. (30), we know that for every $(\varphi, g) \in V \times C^{\infty}(M)$ the map from $V \times C^{\infty}(M)$ to $\mathcal{D}(K)$ defined by

$$
(\varphi, g) \mapsto \int_{M} F_{\varphi}^{(2)}(x, \cdot) g(x) d x=\sum_{|\alpha| \leqslant k}(-1)^{|\alpha|} f_{\alpha}(\varphi, .) \partial^{\alpha} g(.)
$$

is smooth. Choosing $g$ to be equal to the Fourier oscillatory function $e^{-i\langle\xi \cdot x\rangle}$, we obtain by the chain rule the maps that send $(\varphi, \xi)$ to

$$
\int_{M} F_{\varphi}^{(2)}(x, y) e^{-i\langle\xi \cdot x\rangle} d y=\int_{M} \sum_{|\alpha| \leqslant k}(-1)^{|\alpha|} f_{\alpha}(\varphi, y) \delta(x-y) \partial_{y}^{\alpha} e^{-i\langle\xi \cdot x\rangle} d y=\sum_{|\alpha| \leqslant k}(-1)^{|\alpha|} f_{\alpha}(\varphi, x)(-i \xi)^{\alpha}
$$

is Bastiani smooth. Moreover, since the image of the map in Eq. (30) is in $\mathcal{D}(K)$ for every smooth $g$, we obtain that $\sum_{|\alpha| \leqslant k}(-1)^{|\alpha|} f_{\alpha}(\varphi, \cdot)(-i \xi)^{\alpha}$ is in $\mathcal{D}(K)$ for every $\xi$. This is only possible if $f_{\alpha}(\varphi) \in \mathcal{D}(K)$ for every $|\alpha| \leqslant k$. Therefore $\varphi \mapsto f_{\alpha}(\varphi)=\left.\left(i \frac{d}{d \xi}\right)^{\alpha} \int_{M} F_{\varphi}^{(2)}(., y) e^{-i\langle\xi \cdot y\rangle} d y\right|_{\xi=0}$ is Bastiani smooth from $V$ to $\mathcal{D}(K)$ and the proof of the direct sense is complete.

Conversely, we want to prove that if there is a neighborhood $V$ of $\varphi_{0}$, a compact $K \subset M$, and a finite family of smooth maps $\varphi \mapsto f_{\alpha}(\varphi) \in \mathcal{D}(K),|\alpha| \leqslant k$, such that in any system of local coordinates $(x, y)$ on $M^{2}$,

$$
F_{\varphi}^{(2)}(x, y)=\sum_{|\alpha| \leqslant k} f_{\alpha}(\varphi)(x) \partial_{y}^{\alpha} \delta(x-y),
$$

then $\varphi \mapsto \nabla F_{\varphi} \in \mathcal{D}(M)$ is Bastiani smooth. Without loss of generality, we assume that $V$ is convex. By the Taylor formula with remainder for Bastiani smooth functions, for every $\left(\varphi, \psi_{1}, \psi_{2}\right) \in V \times$ $C^{\infty}(M)^{2}$

$$
\begin{aligned}
D^{2} F_{\varphi+s_{1} \psi_{1}+s_{2} \psi_{2}}\left(\psi_{1}, \psi_{2}\right) & =\partial_{s_{1}} \partial_{s_{2}} F\left(\varphi+s_{1} \psi_{1}+s_{2} \psi_{2}\right) \\
& =\partial_{s_{2}} D F_{\varphi+s_{1} \psi_{1}+s_{2} \psi_{2}}\left(\psi_{1}\right),
\end{aligned}
$$

for $s_{1}$ and $s_{2}$ small enough. It follows by the fundamental theorem of calculus and by evaluating at $s_{1}=0$ the previous relation that

$$
\begin{aligned}
D F_{\varphi+t \psi_{2}}\left(\psi_{1}\right) & =D F_{\varphi}\left(\psi_{1}\right)+\int_{0}^{t} \partial_{s_{2}} D F_{\varphi+s_{2} \psi_{2}}\left(\psi_{1}\right) d s_{2} \\
& =D F_{\varphi}\left(\psi_{1}\right)+\int_{0}^{t} D^{2} F_{\varphi+s \psi_{2}}\left(\psi_{1}, \psi_{2}\right) d s
\end{aligned}
$$


where by assumption $D F_{\varphi}\left(\psi_{1}\right)$ is represented by integration against a smooth function

$$
\begin{aligned}
D F_{\varphi}\left(\psi_{1}\right) & =\int_{M} \nabla F_{\varphi}(x) \psi_{1}(x) d x, \\
D^{2} F_{\varphi+s \psi_{2}}\left(\psi_{1}, \psi_{2}\right) & =\int_{M \times M} F_{\varphi+s \psi_{2}}^{(2)}(x, y) \psi_{1}(x) \psi_{2}(y) d x d y,
\end{aligned}
$$

and $F_{\varphi+s \psi}^{(2)}$ is supported on a subset of the diagonal $D_{2} \subset M \times M$ that can be identified with $K$. Hence, for $\psi \in C^{\infty}(M)$ such that $\varphi+\psi \in V$,

$$
\begin{aligned}
\nabla F_{\varphi+\psi}(x) & =\nabla F_{\varphi}(x)+\int_{0}^{1}\left(\int_{M} F_{\varphi+s \psi}^{(2)}(x, y) \psi(y) d y\right) d s \\
& =\nabla F_{\varphi}(x)+\sum_{|\alpha| \leqslant k}(-1)^{|\alpha|} \partial^{\alpha} \psi(x) \int_{0}^{1} f_{\alpha}(\varphi+s \psi)(x) d s .
\end{aligned}
$$

To show that the map $\chi: V \rightarrow \mathcal{D}(K)$ defined by $\chi(\psi)=\nabla F_{\varphi+\psi}$ is smooth, we notice that, according to the last equation, $\nabla F_{\varphi+\psi}$ is the sum of the constant (i.e. independent of $\left.\psi\right) \nabla F_{\varphi}$ and a finite linear combination of products of $\psi \mapsto \partial^{\alpha} \psi$ by an integral over $s$. The integrand $f_{\alpha}(\varphi+s \psi)$ is smooth by assumption. Therefore, the map

$$
\psi \in(V-\varphi) \mapsto \int_{0}^{1} f_{\alpha}(\varphi+s \psi)(x) d s \in \mathcal{D}(K)
$$

is smooth by Lemma VI.11 and the fact that the topology induced on $\mathcal{D}(K)$ by the topology of $C^{\infty}(M)$ is the standard topology of $\mathcal{D}(K)$. The map $\psi \mapsto \partial^{\alpha} \psi$ is smooth because it is linear and continuous. Finally, the product of the integral by $\partial^{\alpha} \psi$ is smooth by a trivial extension of Lemma VI.15. This completes the proof of Lemma VI.9.

We are now ready to prove Theorem VI.3 characterizing local functionals.

\section{Proof of Theorem VI.3}

Let us start by proving the converse where we assume that $F$ is the integral of some local function on jet space. Let $\varphi \in U$ and $V$ be some neighborhood of $\varphi$ such that $F(\varphi+\psi)=\int_{M} f\left(x, j_{x}^{k} \psi\right) d x$ for every $\psi \in V$ where $j_{x}^{k} \psi$ is the $k$-jet of $\psi$ at $x$ and where $f$ is smooth and compactly supported in the variable $x$ in some fixed compact $K \subset M$. Without loss of generality, we can restrict the support $K$ of $f$ by a smooth partition of unity and assuming that $K$ is contained in some open chart of $M$, we may reduce to the same problem for $f \in C^{\infty}(\Omega)$ where $\Omega$ is some open set in $\mathbb{R}^{d}$ and $K \subset \Omega$.

We choose a smooth compactly supported function $\chi \in \mathcal{D}(\Omega)$ such that $\chi=1$ on a compact neighborhood of $K$ with supp $\chi \subset \Omega$ and we observe that

$$
\Psi: \psi \in C^{\infty}(\Omega) \longmapsto\left(\partial^{\alpha} \psi\right)_{|\alpha| \leqslant k} \chi \in \mathcal{D}(\operatorname{supp}(\chi))^{\frac{(d+k) !}{d !}}
$$

is linear continuous hence Bastiani smooth. We need a simple

Lemma VI.12. Let $\Omega$ be an open set in $\mathbb{R}^{d}$ then the map

$$
\Phi: \varphi \in C^{\infty}\left(\Omega, \mathbb{R}^{r}\right) \mapsto\{x \mapsto(x, \varphi(x))\} \in C^{\infty}\left(\Omega, \mathbb{R}^{d} \times \mathbb{R}^{r}\right)
$$

is Bastiani smooth.

Proof. The first Bastiani differential $D \Phi_{\varphi}(h)$ can be identified with the smooth function $x \mapsto(0$, $h(x)$ ), which is linear continuous in $h$ and does not depend on $\varphi$. It is thus smooth and so is $\Phi$. 
Therefore, the composition

$$
\Phi \circ \Psi: C^{\infty}(\Omega) \rightarrow C^{\infty}\left(\Omega, \mathbb{R}^{d} \times \mathbb{R}^{\frac{(d+k) !}{d !}}\right)
$$

defined by

$$
\Phi \circ \Psi(\psi): x \mapsto\left(x, \chi \partial^{\alpha} \psi(x)_{|\alpha| \leqslant k}\right)
$$

is Bastiani smooth and finally

$$
\psi \in C^{\infty}(M) \mapsto f\left(., j_{x}^{k} \psi(.)\right) \in \mathcal{D}(K) \mapsto \int_{\Omega} f\left(x, j_{x}^{k} \psi\right) d x
$$

is Bastiani smooth by the chain rule, and since the last integration map is linear continuous thus Bastiani smooth.

Now let us prove the direct sense of Theorem VI.3, where we start from a functional characterization of $F$ and end up with a representation as a function $F(\varphi+\psi)=\int_{M} f\left(x, j_{x}^{k} \psi\right) d x$ on jet space, for $\varphi+\psi$ in a neighborhood $V$ of $\varphi$, that we assume convex. We start by deriving a candidate for the function $f$. According to the fundamental theorem of calculus,

$$
F(\varphi+\psi)=F(\varphi)+\int_{0}^{1} d t D F_{\varphi+t \psi}(\psi) .
$$

As discussed at the beginning of this section, since we assume that $\operatorname{WF}\left(F_{\varphi}^{(1)}\right)=\emptyset$ for every $\varphi \in U$, there exists a unique smooth compactly supported function $x \mapsto \nabla F_{\varphi}(x)$ such that

$$
F_{\varphi}^{(1)}(\psi)=\int_{M} d x \nabla F_{\varphi}(x) \psi(x)
$$

Therefore Eq. (31) reads

$$
F(\varphi+\psi)=F(\varphi)+\int_{0}^{1} d t \int_{M} \nabla F_{\varphi+t \psi}(x) \psi(x) d x
$$

We show that Fubini's theorem can be applied to the function $\chi:(x, t) \mapsto \nabla_{\varphi+t \psi} F(x) \psi(x)$. By Proposition III.4, $F^{(1)}$ is locally compactly supported so that there is a convex neighborhood $V$ of $\varphi$ and a compact subset $K$ of $\Omega$ such that $F_{\varphi+\psi}^{(1)}$ is supported in $K$ for every $\varphi+\psi \in V$. The function $\chi$ is defined on $[0,1] \times K$ and supported on $K$ for fixed $t \in[0,1]$. Moreover, by imposing the additional assumption carried by item 2 in Theorem VI.3, namely, that $\varphi \mapsto \nabla F_{\varphi}$ be Bastiani smooth from $U$ to $\mathcal{D}(M)$, the support property of $F$ implies that the image of $\nabla F_{\varphi+t \psi}$ is actually in $\mathcal{D}(K)$ and $\nabla F$ is smooth from $V$ to $\mathcal{D}(K)$ because the topology induced on $\mathcal{D}(K)$ by $\mathcal{D}(M)$ is the Fréchet topology of $\mathcal{D}(K)$ determined by the seminorms $\pi_{m, K}$ (Ref. 30, p. 172). Since $\mathcal{D}(K)$ injects continuously in $\left(C^{0}(K), \pi_{0, K}\right), \varphi \mapsto$ $D_{\varphi} F$ is a continuous $\left(C^{0}(K), \pi_{0, K}\right)$-valued map. This implies that $(t, x) \mapsto \nabla F_{\varphi+t \psi}(x)$ is continuous as a $\mathbb{K}$-valued function on $[0,1] \times K$. Hence so is the integrand of (33), and the Fubini theorem holds and we obtain

Lemma VI.13. Let U be an open subset of $E=C^{\infty}(M)$ and $F: E \rightarrow \mathbb{K}$ be Bastiani smooth. Assume that for every $\varphi \in U, \operatorname{WF}\left(F_{\varphi}^{(1)}\right)=\emptyset$ and $F^{(1)}: U \rightarrow \mathcal{D}(M)$ is Bastiani smooth, then, for every $\varphi \in U$, there is a convex neighborhood $V$ of $\varphi$ such that if $\varphi+\psi \in V$, then

$$
F(\varphi+\psi)=F(\varphi)+\int_{M} d x \int_{0}^{1} \nabla F_{\varphi+t \psi}(x) \psi(x) d t .
$$

From now on, we consider $\varphi \in U$ to be fixed. Our candidate for $f\left(j_{x}^{k} \psi\right)$ is

$$
c_{\psi}(x)=\int_{0}^{1} \nabla F_{\varphi+t \psi}(x) d t \psi(x)
$$

By definition and Lemma VI.13, for all $\psi$ such that $\varphi+\psi \in V$,

$$
F(\varphi+\psi)=F(\varphi)+\int_{M} c_{\psi}(x) d x .
$$

To show that $c_{\psi}(x)$ is the right candidate, we first need 
Proposition VI.14. The function $c_{\psi}$ depends only on a finite jet of $\psi$. More precisely, for every $\varphi$ $\in U$, if there is a convex neighborhood $V$ of $\varphi$ and an integer $k \geq 0$ such that, for all $x \in M$, for every $\psi_{1}$ and $\psi_{2}$ such that $\varphi+\psi_{1}$ and $\varphi+\psi_{2}$ are in $V$ and $j_{x}^{k} \psi_{1}=j_{x}^{k} \psi_{2}$, then $c_{\psi_{1}}(x)=c_{\psi_{2}}(x)$.

The beginning of the proof is inspired by Ref. 20. For fixed $\varphi \in U$, by Proposition III.11, there exist an integer $k$, a compact $K$, and a convex neighborhood $V$ of $\varphi$ such that the order of $F_{\varphi+\psi}^{(2)}$ is smaller than $k$ and the support of $D F_{\varphi+\psi}$ is in $K$ if $\varphi+\psi \in V$.

Let us choose some point $x_{0} \in M$. Consider a pair $\psi_{1}, \psi_{2}$ of smooth functions such that $\psi_{1}\left(x_{0}\right)$ $=\psi_{2}\left(x_{0}\right)$. Then,

$$
\begin{aligned}
c_{\psi_{1}}\left(x_{0}\right)-c_{\psi_{2}}\left(x_{0}\right) & =\int_{0}^{1} d t\left(\nabla F_{\varphi+t \psi_{2}}\left(x_{0}\right) \psi_{2}\left(x_{0}\right)-\nabla F_{\varphi+t \psi_{1}}\left(x_{0}\right) \psi_{1}\left(x_{0}\right)\right) \\
& =\psi_{1}\left(x_{0}\right) \int_{0}^{1} d t\left(\nabla F_{\varphi+t \psi_{2}}\left(x_{0}\right)-\nabla F_{\varphi+t \psi_{1}}\left(x_{0}\right)\right) .
\end{aligned}
$$

We use the fundamental theorem of analysis again for $D F_{\varphi}(h)=\int_{M} d x \nabla F_{\varphi}(x) h(x)$ for an arbitrary $h \in C^{\infty}(M)$ to get

$$
D F_{\varphi+t \psi_{2}}(h)-D F_{\varphi+t \psi_{1}}(h)=t \int_{0}^{1} d s\left\langle F_{\varphi+t \psi_{1}+s t\left(\psi_{2}-\psi_{1}\right)}^{(2)},\left(\psi_{2}-\psi_{1}\right) \otimes h\right\rangle
$$

Now we take a sequence of smooth functions $\left(h_{n}\right)_{n \in \mathbb{N}}$ which converges to $\delta_{x_{0}}$ in $\mathcal{D}^{\prime}(M)$ when $n$ goes to infinity and show that both the left- and right-hand sides have limits. For the left-hand side, the distribution $D F_{\varphi+t \psi_{i}}$ being smooth, it defines the continuous form $u \mapsto D F_{\varphi+t \psi_{i}}(u)$ on $\mathcal{D}^{\prime}(M)$ by duality pairing. By continuity, $D F_{\varphi+t \psi_{i}}\left(h_{n}\right) \rightarrow D F_{\varphi+t \psi_{i}}\left(\delta_{x_{0}}\right)$ and Eq. (32) yields

$$
\begin{aligned}
h & =D F_{\varphi+t \psi_{2}}\left(\delta_{x_{0}}\right)-D F_{\varphi+t \psi_{1}}\left(\delta_{x_{0}}\right) \\
& =\int_{M} d x\left(\nabla F_{\varphi+t \psi_{2}}(x)-\nabla F_{\varphi+t \psi_{1}}^{(1)}(x)\right) \delta\left(x-x_{0}\right) \\
& =\nabla F_{\varphi+t \psi_{2}}\left(x_{0}\right)-\nabla F_{\varphi+t \psi_{1}}\left(x_{0}\right) .
\end{aligned}
$$

For the right-hand side, we know by Lemma VI.9 that for every $s \in[0,1]$, the wave front set of the distribution $F_{\varphi+t \psi_{1}+s t\left(\psi_{2}-\psi_{1}\right)}^{(2)}$ is in the conormal $C_{2}$ and the sequence $\left(\psi_{2}-\psi_{1}\right) \otimes h_{n}$ converges to $\left(\psi_{2}-\psi_{1}\right) \otimes \delta_{x_{0}}$ in $\mathcal{D}_{N^{*}\left(M \times\left\{x_{0}\right\}\right)}^{\prime}$, where $N^{*}\left(M \times\left\{x_{0}\right\}\right)$ is the conormal of the submanifold $M \times\left\{x_{0}\right\} \subset$ $M \times M$ in $T^{*}(M \times M)$. Therefore, by transversality of the wave front sets and hypocontinuity of the duality pairings, ${ }^{106}$ the following limit exists:

$$
\lim _{n}\left\langle F_{\varphi+t \psi_{1}+s t\left(\psi_{2}-\psi_{1}\right)}^{(2)},\left(\psi_{2}-\psi_{1}\right) \otimes h_{n}\right\rangle .
$$

Moreover, still by Lemma VI.9, we have for $\varphi+\psi \in V$,

$$
\left\langle F_{\varphi+\psi}^{(2)}, g \otimes h\right\rangle=\sum_{|\alpha| \leq k}(-1)^{|\alpha|} \int_{M} d x \theta_{\psi}^{\alpha}(x) g(x) \partial^{\alpha} h(x),
$$

for every $(g, h) \in C^{\infty}(M)^{2}$ and all $\theta_{\psi}^{\alpha}$ belong to $\mathcal{D}(K)$. An integration by parts yields

$$
\left\langle F_{\varphi+\psi}^{(2)}, g \otimes h\right\rangle=\sum_{|\alpha| \leq k} \int_{M} d x f_{\psi}^{\alpha}(x) h(x) \partial^{\alpha} g(x),
$$

where $f_{\psi}^{\alpha}=\sum_{\beta}\left(\begin{array}{l}\beta \\ \alpha\end{array}\right) \partial^{\beta-\alpha} \theta_{\psi}^{\beta}$ and the sum is over the multi-indices such that $\beta \geq \alpha$ and $|\beta| \leqslant k$. 
As a consequence, for $\varphi+\psi_{1}+\psi_{2}$ in the convex neighborhood $V$,

$$
X=\left\langle F_{\varphi+t \psi_{1}+s t\left(\psi_{2}-\psi_{1}\right)}^{(2)},\left(\psi_{2}-\psi_{1}\right) \otimes \delta_{x_{0}}\right\rangle=\sum_{|\alpha| \leq k} f_{t \psi_{1}+s t\left(\psi_{2}-\psi_{1}\right)}^{\alpha}\left(x_{0}\right) \partial^{\alpha}\left(\psi_{2}-\psi_{1}\right)\left(x_{0}\right) .
$$

If, at the point $x_{0}, j_{x_{0}}^{k} \psi_{1}=j_{x_{0}}^{k} \psi_{2}$, then

$$
c_{\psi_{1}}\left(x_{0}\right)-c_{\psi_{2}}\left(x_{0}\right)=\psi_{1}\left(x_{0}\right) \sum_{|\alpha| \leq k} \int_{0}^{1} t d t \int_{0}^{1} d s f_{t \psi_{1}+s t\left(\psi_{2}-\psi_{1}\right)}^{\alpha}\left(x_{0}\right)\left(\partial^{\alpha} \psi_{2}\left(x_{0}\right)-\partial^{\alpha} \psi_{1}\left(x_{0}\right)\right)=0 .
$$

We showed that $c_{\psi}$ depends only on the $k$-jet of $\psi$ at $x_{0}$. Moreover, the number $k$ depends only on $V$ and not on $x_{0}$ so that $c_{\psi}$ depends on the $k$-jet for every $x \in M$. In other words, there is an integer $k$ and a function $f$ such that $c_{\psi}(x)=f\left(x, \psi(x), \ldots, \partial^{\alpha} \psi(x)\right)$ for every $x \in M$, where $1 \leq|\alpha| \leq k$.

We want to show that $f$ is smooth in its arguments, hence we now investigate in which manner $c_{\psi}$ depends on $\psi$. This suggests to study the regularity of the $\mathcal{D}(K)$-valued function $\psi \mapsto \nabla F_{\varphi+t \psi} \psi$. More precisely, we need to show that the map $\psi \mapsto \nabla F_{\varphi+t \psi} \psi$ is Bastiani smooth from $U$ to $\mathcal{D}(K)$. This is not completely trivial because the map $x \mapsto \nabla F_{\varphi+t \psi}(x)$ is in $\mathcal{D}(K)$ and $\psi$ is in $C^{\infty}(M)$ and we must check that the product of a function in $\mathcal{D}(K)$ by a function in $C^{\infty}(M)$ is continuous (Ref. 27, p. 119).

Lemma VI.15. If U is an open set in $C^{\infty}(M)$ and $F: U \rightarrow \mathcal{D}(K)$ is a compactly supported Bastianismooth map, then the function $G: U \rightarrow \mathcal{D}(M)$ defined by $G(\varphi)=F(\varphi) \varphi$ is compactly supported Bastiani-smooth with the same support as $F$.

Proof. Dabrowski pointed out to us the following fact. For any compact subset $K$ of $\Omega$, both $\mathcal{D}(\Omega)$ and $C^{\infty}(\Omega)$ induce on $\mathcal{D}(K)$ the usual Fréchet topology of $\mathcal{D}(K)$ (Ref. 30, p. 172). Thus to establish the smoothness of $G$, it suffices to show that the multiplication $(u, v) \in \mathcal{D}(K) \times C^{\infty}(M) \mapsto \mathcal{D}(K)$ is continuous and then it would be Bastiani smooth, and by the chain rule it follows that $\varphi \mapsto(F(\varphi), \varphi) \mapsto$ $F(\varphi) \varphi$ is smooth. Since both $\mathcal{D}(K)$ and $C^{\infty}(M)$ are Fréchet, the product $\mathcal{D}(K) \times C^{\infty}(M)$ endowed with the product topology is metrizable and it is enough to prove that the product is sequentially continuous. Indeed, let $\left(u_{n}, v_{n}\right) \rightarrow(u, v)$ in $\mathcal{D}(K) \times C^{\infty}(M)$, we can find some cutoff function $\chi \in \mathcal{D}(M)$ such that $\chi=1$ on the support of all $u_{n}$, and for all $m$, by (Ref. 85, p. 1351)

$$
\begin{aligned}
\pi_{m, K}\left(u v-u_{n} v_{n}\right) & \leqslant \pi_{m, K}\left(\left(u-u_{n}\right) v \chi\right)+\pi_{m, K}\left(u_{n} \chi\left(v-v_{n}\right)\right) \\
& \leqslant 2^{m}\left(\pi_{m, K}\left(u-u_{n}\right) \pi_{m, K}(\chi v)+\pi_{m, K}\left(u_{n}\right) \pi_{m, K}\left(\left(v-v_{n}\right) \chi\right)\right) \rightarrow 0 .
\end{aligned}
$$

Hence $G$ is smooth.

This implies that $\psi \mapsto c_{\psi}=\int_{0}^{1} \nabla F_{\varphi+t \psi} \psi d t$ is smooth since the above lemma shows the smoothness of $a(t, \psi) \mapsto \nabla F_{\varphi+t \psi} \psi$ and integration over $t$ conserves smoothness by Lemma VI.11. At this point, Theorem VI.3 follows directly from Proposition VI.4.

\section{Representation theory of local functionals}

In this section, we discuss the issue of representation of our local functionals and the relations between the functionals $\left(c_{\psi}, \nabla F, f\left(j_{x}^{k} \psi\right)\right)$ which are defined or constructed in the course of our proof of Theorem VI.3. In the sequel, we assume that our manifold $M$ is connected, oriented without boundary, hence we can fix a density $d x$ on $M$ which is also a differential form on $M$ of top degree.

In the sequel, we shall work out all explicit formulas in local charts which means without loss of generality that we work on $\mathbb{R}^{d}$, and the reference density $d x$ is chosen to be the standard Lebesgue measure. We will denote by $\left(x, u, u^{\alpha}\right)_{|\alpha| \leqslant k}$ where $\alpha$ are multi-indices, some local coordinates on the jet bundle $J^{k}\left(\mathbb{R}^{d}\right)$. Introduce the vertical Euler vector field $\rho=\sum u^{(\alpha)} \frac{\partial}{\partial u^{(\alpha)}}$ on the bundle $J^{k}\left(\mathbb{R}^{d}\right)$. In the manifold case if we work on $J^{k}(M)$, this vector field is intrinsic since it generates scaling in the fibers of $J^{k}(M)$. For all multi-index $(\alpha)=\left(\alpha_{1} \ldots \alpha_{p}\right), \alpha_{i} \in\{1, \ldots, d\}$, introduce the operators $\partial^{(\alpha)}=\partial^{\alpha_{1}} \ldots \partial^{\alpha_{p}}$ where $\partial^{i}=\frac{\partial}{\partial x^{i}}+\sum_{\alpha} u^{(\alpha i)} \frac{\partial}{\partial u^{(\alpha)}}$ and the Euler-Lagrange operator $E L=u \frac{\partial}{\partial u}+\sum_{\alpha}(-1)^{|\alpha|} \partial^{(\alpha)} u^{(\alpha)} \frac{\partial}{\partial u^{(\alpha)}}$. 
Let us discuss the nature of the objects involved, $\rho$ is a vertical vector field and acts on $C^{\infty}\left(J^{k} M\right)$ as a $C^{\infty}(M)$ linear map, for $\chi \in C^{\infty}\left(\mathbb{R}^{d}\right), f \in C^{\infty}\left(J^{k} \mathbb{R}^{d}\right), \rho(\chi f)=\chi(\rho f)$. For every $i \in\{1, \ldots, d\}, \partial^{i}$ is a vector field on $J^{k} \mathbb{R}^{d}$ but it has a horizontal component; therefore, it is not $C^{\infty}\left(\mathbb{R}^{d}\right)$ linear and the Euler-Lagrange operator is not $C^{\infty}\left(\mathbb{R}^{d}\right)$ linear either.

What follows is a definition-proposition-where we give an intrinsic and global definition of the Euler-Lagrange operator in terms of the operator $\nabla F$ associated with a functional.

Proposition VI.16 (Euler-Lagrange operator is intrinsic). Let $U$ be an open subset of $C^{\infty}(M)$ and $F: U \rightarrow \mathbb{K}$ be a Bastiani smooth local functional. For $\varphi \in U$, if there is an integer $k$, a neighborhood $V$ of $\varphi$, an open subset $\mathcal{V}$ of $J^{k} M$, and $f \in C^{\infty}(\mathcal{V})$ such that $x \mapsto f\left(j_{x}^{k} \psi\right)$ is compactly supported and

$$
F(\varphi+\psi)=F(\varphi)+\int_{M} f\left(j_{x}^{k} \psi\right) d x
$$

whenever $\varphi+\psi \in V$, then in every local chart

$$
\nabla F_{\varphi}=E L(f)\left(j^{k} \psi\right)
$$

where $E L(f)(\psi)=\sum_{|\alpha| \leqslant k}(-1)^{|\alpha|}\left(\partial^{(\alpha)}\left(\frac{\partial f}{\partial u^{(\alpha)}}\right)\right)\left(j_{x}^{k} \psi\right)$ and $E L(f)(\psi)$ is uniquely determined by $F$.

The above proposition means that $E L(f)$ does not depend on the choice of representative $f$ and is intrinsic (i.e., it does not depend on the choice of a local chart).

Proof. Indeed, assume that we make a small perturbation $\varphi+\psi$ of the background field $\varphi$ by $\psi$ which is compactly supported in some open chart $U$ of $M$. Then a local calculation yields

$$
\begin{aligned}
D F_{\varphi}(\psi) & =\sum_{\alpha} \int_{M} \frac{\partial f}{\partial u^{(\alpha)}(x)} \psi^{(\alpha)}(x) d x \\
& =\sum_{|\alpha| \leq k}(-1)^{|\alpha|} \int_{M} \psi(x)\left(\partial^{(\alpha)}\left(\frac{\partial f}{\partial u^{(\alpha)}}\right)\right) d x,
\end{aligned}
$$

where we used an integration by parts to recover the Euler-Lagrange operator and all boundary terms vanish since $f$ is compactly supported in $x$ and $\psi \in \mathcal{D}(U)$. We have just proved that for all open charts $U \subset M,\left.\nabla F_{\varphi}\right|_{U}=\left.E L(f)\right|_{U}$. But $\nabla F_{\varphi}$ is intrinsically defined on $M$ therefore so is $E L(f)$ and we have the equality $\nabla F=E L(f)$. The unique determination of $\nabla F_{\varphi}$ follows from Lemma VI.2.

Theorem VI.17 [Global Poincaré]. Assume that $M$ is a smooth, connected, oriented manifold without boundary. Let $U$ be an open subset of $C^{\infty}(M)$ and $F: U \rightarrow \mathbb{K}$ be a Bastiani smooth local functional. Then the following statements are equivalent:

- Two functions, $\left(f_{1}, f_{2}\right) \in C^{\infty}(\mathcal{V})$ for $\mathcal{V}$ an open subset of the jet space $J^{k} M$, are two representations of $F$ in a neighborhood $V$ of $\varphi \in U$,

$$
F(\varphi+\psi)=F(\varphi)+\int_{M} f_{1}\left(j_{x}^{k} \psi\right) d x=F(\varphi)+\int_{M} f_{2}\left(j_{x}^{k} \psi\right) d x,
$$

whenever $\varphi+\psi \in V$.

- For all $\psi \in V-\varphi$,

$$
f_{1}\left(j_{x}^{k} \psi\right) d x-f_{2}\left(j_{x}^{k} \psi\right) d x=d \beta\left(j_{x}^{2 k} \psi\right),
$$

where $\beta\left(j_{x}^{2 k} \psi\right) \in \Omega_{c}^{d-1}(M)$ is a differential form of degree $d-1$ whose value at a point $x$ depends only on the $2 k$-jet of $\psi$ at $x$. 
Let us stress that we do not need to constraint the topology of $M$ in the above theorem and only the compactness of the support of $f_{i}\left(j_{x}^{k} \psi\right) d x, i \in\{1,2\}$ really matters.

Proof. One sense of the equivalence is trivial since the integral of a compactly supported exact form on $M$ always vanishes. By Proposition VI.4, we know that the map $\psi \in V-\varphi \mapsto j^{k} \psi$ has an open image in $J^{k}(M)$ denoted $\mathcal{V}$ and we only need $\left(f_{1}, f_{2}\right)$ to be defined on $\mathcal{V}$. Recall we denote by $\left(x, u, u^{\alpha}\right)_{|\alpha| \leqslant k}$ where $\alpha$ are multi-indices, some local coordinates on the jet bundle $J^{k}\left(\mathbb{R}^{d}\right)$. We use the vertical Euler vector field $\rho=\sum u^{(\alpha)} \frac{\partial}{\partial u^{(\alpha)}}$ on the bundle $J^{k}\left(\mathbb{R}^{d}\right)$. For every multi-index $(\alpha)=\left(\alpha_{1} \ldots \alpha_{p}\right), \alpha_{i} \in\{1, \ldots, d\}$, introduce the operators $\partial^{(\alpha)}=\partial^{\alpha_{1}} \ldots \partial^{\alpha_{p}}$ where $\partial^{i}=\frac{\partial}{\partial x^{i}}+$ $\sum_{\alpha} u^{(\alpha i)} \frac{\partial}{\partial u^{(\alpha)}}$ and the Euler-Lagrange operator reads $E L=u \frac{\partial}{\partial u}+\sum_{\alpha}(-1)^{|\alpha|} \partial^{(\alpha)} u^{(\alpha)} \frac{\partial}{\partial u^{(\alpha)}}$.

We shall prove two related identities, in local chart,

$$
\left.(\rho f)\left(j^{k} \psi\right) d x=(u E L(f))\left(j^{k} \psi\right) d x+d\left(\sum_{\mu=1}^{d} j_{\mu}\left(j^{2 k} \psi\right) \frac{\partial}{\partial x^{\mu}}\right\lrcorner d x\right)
$$

$\left.f\left(j^{k}\left(\psi_{1}+\psi_{2}\right)\right) d x=f\left(j^{k} \psi_{1}\right) d x+\int_{0}^{1} d t \psi_{2} E L(f)\left(j^{k}\left(\psi_{1}+t \psi_{2}\right)\right) d x+d\left(\int_{0}^{1} \frac{d t}{t} j_{\mu}\left(j^{2 k}\left(\psi_{1}+t \psi_{2}\right)\right) \partial_{x^{\mu}}\right\lrcorner d x\right)$.

For all $(f, g) \in C^{\infty}\left(J^{k} \mathbb{R}^{d}\right)^{2}$ and all multi-indices $\alpha$, the generalized Leibniz-like identity holds true,

$$
\left(\partial^{\alpha_{1}} \ldots \partial^{\alpha_{p}} f\right) g=(-1)^{p} f\left(\partial^{\alpha_{p}} \ldots \partial^{\alpha_{1}} g\right)+\sum_{i=1}^{p}(-1)^{i+1} \partial^{\alpha_{i}}\left(\left(\partial^{\alpha_{i+1}} \ldots \partial^{\alpha_{p}} f\right) \partial^{\alpha_{i-1}} \ldots \partial^{\alpha_{1}} g\right)
$$

where the second term is a sum of total derivatives. Using this, we derive the following key identity which is valid on jet spaces. For all $f d x \in C^{\infty}\left(J^{k} \mathbb{R}^{d}\right) \otimes \Omega^{d}\left(\mathbb{R}^{d}\right)$,

$$
\begin{aligned}
(\rho f) d x & =\sum u^{(\alpha)} \frac{\partial f}{\partial u^{(\alpha)}} d x=u \frac{\partial f}{\partial u}+\sum_{|\alpha| \geqslant 1} u^{(\alpha)} \frac{\partial f}{\partial u^{(\alpha)}} d x \\
& =u E L(f) d x+\sum_{\mu=1}^{d} \partial^{\mu} j_{\mu}\left(j^{2 k} \psi\right) d x \\
& \left.=u E L(f) d x+d\left(\sum_{\mu=1}^{d} j_{\mu}\left(j^{2 k} \psi\right) \frac{\partial}{\partial_{x^{\mu}}}\right\lrcorner d x\right),
\end{aligned}
$$

where $j_{\mu} \in C^{\infty}\left(J^{2 k} \mathbb{R}^{d}\right)$ is a local functional.

To prove the second identity, we shall use the fundamental theorem of calculus and the first identity,

$$
\begin{aligned}
f\left(j^{k}\left(\psi_{1}+\psi_{2}\right)\right) d x & =f\left(j^{k} \psi_{1}\right) d x+\int_{0}^{1} \frac{d t}{t}(\rho f)\left(j^{k}\left(\psi_{1}+t \psi_{2}\right)\right) d x \\
& \left.=f\left(j^{k} \psi_{1}\right) d x+\int_{0}^{1} d t \psi_{2} E L(f)\left(j^{k}\left(\psi_{1}+t \psi_{2}\right)\right) d x+d\left(\int_{0}^{1} \frac{d t}{t} j_{\mu}\left(j^{2 k}\left(\psi_{1}+t \psi_{2}\right)\right) \partial_{x^{\mu}}\right\lrcorner d x\right) .
\end{aligned}
$$

To prove the claim of the lemma is equivalent to show the following statement: if a local functional $F$ is locally constant, i.e., $F(\varphi+\psi)=F(\varphi)$ whenever $\varphi+\psi \in V$, then $F(\varphi+\psi)=F(\varphi)+\int_{M} d \beta\left(j_{x}^{2 k} \psi\right)$ and $\beta\left(j_{x}^{2 k} \psi\right) \in \Omega_{c}^{n-1}(M)$ is a compactly supported $n-1$ form. For all $\psi$ in $V-\varphi$,

$$
F(\varphi+t \psi)=F(\varphi) \Longrightarrow \int_{\mathbb{R}^{d}} \int_{0}^{1} \frac{d t}{t}(\rho f)\left(j_{x}^{k}(t \psi)\right) d x=0, \Longrightarrow \int_{\mathbb{R}^{d}} \int_{0}^{1} d t\left(\psi E L(f)\left(j_{x}^{k}(t \psi)\right) d x\right)=0 .
$$


This means that $E L(f)=0$ and therefore on any open chart $U$ ( $U$ is contractible), Eq. (39) yields

$$
\left.f\left(j_{x}^{p}(\psi)\right)=f(0)+d \int_{0}^{1} d t\left(\sum_{\mu} j_{\mu}\left(j_{x}^{2 p}(t \psi)\right) \partial_{x^{\mu}}\right\lrcorner d x\right) .
$$

We want to prove that $E L(f)=0 \Longrightarrow f\left(j_{x}^{k}(\psi)\right) d x-\left.f(0) d x\right|_{M_{p+1}}=d \beta\left(j^{2 k} \psi\right)$, where $\beta \in C^{\infty}\left(J^{2 p}(M)\right)$ knowing that this holds true on any local chart and that $E L(f)=0$ is equivalent to assuming that $F(\varphi+$ $\psi):=\int_{M} f\left(j_{x}^{k} \psi\right) d x$ is locally constant. We cover $M$ by some countable union $\cup_{i \in \mathbb{N}} U_{i}$ of contractible open charts such that every element $x \in M$ belongs to a finite number of charts $U_{i}$, set $M_{p}=$ $\left(U_{1} \cup \cdots \cup U_{p}\right)$, and arrange the cover in such a way that $M_{p} \cap U_{p+1} \neq \emptyset$ for all $p$ which is always possible. Assume by induction on $p$ that $E L(f)=0$ and $\operatorname{supp}(f) \subset M_{p}$ implies

$$
f\left(j_{x}^{k}(\psi)\right) d x-\left.f(0) d x\right|_{M_{p}}=d \beta\left(j^{2 k} \psi\right),
$$

where $\beta \in C^{\infty}\left(J^{2 p}(M)\right) \otimes \Omega_{c}^{d-1}\left(M_{p}\right)$.

We want to prove that $E L(f)=0$, supp $(f) \subset M_{p+1} \Longrightarrow f\left(j_{x}^{k}(\psi)\right) d x-\left.f(0) d x\right|_{M_{p+1}}=d \beta\left(j^{2 k} \psi\right)$, where $\beta \in C^{\infty}\left(J^{2 p}(M)\right) \otimes \Omega_{c}^{d-1}\left(M_{p+1}\right)$. Choosing a partition of unity $(\chi, 1-\chi)$ subordinated to $M_{p} \cup$ $U_{p+1}$, the key idea is to decompose the variation $\psi$ of the background field $\varphi$ as the sum of two components $\chi \psi+(1-\chi) \psi$ where $\chi \psi($ respectively, $(1-\chi) \psi)$ vanishes outside $U_{p}\left(\operatorname{resp} U_{p+1}\right)$ which yields

$$
f\left(j^{k} \psi\right)=f\left(j^{k}(\chi \psi+(1-\chi) \psi)\right)-f\left(j^{k}((1-\chi) \psi)\right)+f\left(j^{k}((1-\chi) \psi)\right)-f(0)+f(0) .
$$

The second idea is to note that for every fixed $\psi$, the new functional

$$
\phi \mapsto \tilde{f}\left(j^{k} \phi\right)=f\left(j^{k}(\chi \phi+(1-\chi) \psi)\right)-f\left(j^{k}((1-\chi) \psi)\right)
$$

has the trivial Euler-Lagrange equation $E L(\tilde{f})\left(j^{k} \phi\right)=E L(f)\left(j^{k}(\chi \phi+(1-\chi) \psi)\right)=0$ since $E L(f)=0$ and its support is contained in $M_{p}$. Therefore,

$$
\begin{aligned}
f\left(j^{k} \psi\right) & =\tilde{f}\left(j^{k} \psi\right)+f\left(j^{k}((1-\chi) \psi)\right)-f(0)+f(0) \\
& =d \tilde{\beta}\left(j^{2 k} \psi\right)+\underbrace{f\left(j^{k}((1-\chi) \psi)\right)-f(0)}+f(0)
\end{aligned}
$$

by the inductive assumption. To treat the term under brace, define a new functional

$$
\psi \mapsto g\left(j^{k} \psi\right)=f\left(j^{k}((1-\chi) \psi)\right)-f(0),
$$

whose support is contained in $U_{p+1}$ and whose Euler-Lagrange equation vanishes, $E L(g)=0$ again by the fact that $E L(f)=0$. Since $U_{p+1}$ is contractible, we know that $f\left(j^{k}((1-\chi) \psi)\right)-f(0)=d \alpha\left(j^{2 k} \psi\right)$ where $\alpha \in C^{\infty}\left(J^{2 k} M\right) \otimes \Omega_{c}^{d-1}\left(U_{p+1}\right)$ and therefore we found that

$$
f\left(j^{k} \psi\right) d x=d \beta\left(j^{2 k} \psi\right)+f(0) d x,
$$

where $\beta \in C^{\infty}\left(J^{2 k} M\right) \otimes \Omega_{c}^{d-1}\left(M_{p+1}\right)$. Therefore for all $\psi \in V-\varphi, f\left(j_{x}^{k}(\psi)\right) d x=d \beta\left(j^{2 k} \psi\right)+f(0) d x$. Now we conclude by using the fact that $F$ is a constant functional, thus $0=F(\varphi+\psi)-F(\varphi)=$ $\int_{M}\left(f(0) d x+d \beta\left(j^{2 k} \psi\right)\right)=\int_{M} f(0) d x$. But $f(0) d x$ is a top form in $\Omega_{c}^{d}(M)$ which does not depend on $\psi$ and whose integral over $M$ vanishes, hence $f(0) d x=d k$ for some $k \in \Omega_{c}^{d-1}(M)$ since $H_{c}^{d}(M, \mathbb{R}) \simeq \mathbb{R}$ for the top de Rham cohomology with compact support when $M$ is connected (Ref. 107, Theorem 17.30, p. 454).

The next theorem summarizes the above results. 
Theorem VI.18. Let $U$ be an open subset of $C^{\infty}(M)$ and $F: U \rightarrow \mathbb{K}$ be a Bastiani smooth local functional. For $\varphi \in U$, if there is an integer $k$, a neighborhood $V$ of $\varphi$, an open subset $\mathcal{V}$ of $J^{k} M$, and $f \in C^{\infty}(\mathcal{V})$ such that $x \mapsto f\left(j^{k} \psi_{x}\right)$ is compactly supported and

$$
F(\varphi+\psi)=F(\varphi)+\int_{M} f\left(j_{x}^{k} \psi\right) d x
$$

whenever $\varphi+\psi \in V$, then in every local chart

$$
\nabla F_{\varphi}=E L(f)\left(j^{k} \psi\right)
$$

where $\operatorname{EL}(f)(\psi)=\sum_{|\alpha| \leqslant k}(-1)^{|\alpha|}\left(\partial^{(\alpha)}\left(\frac{\partial f}{\partial u^{(\alpha)}}\right)\right)\left(j_{x}^{k} \psi\right)$ is the Euler-Lagrange operator and $E L(f)(\psi)$ is uniquely determined by $F$.

Furthermore, we find that

$$
\begin{aligned}
F(\varphi+\psi) & =F(\varphi)+\int_{M} f\left(j_{x}^{k} \psi\right) d x \\
& =F(\varphi)+\int_{M}\left(\int_{0}^{1} d t \psi E L(f)(t \psi) \psi\right) d x .
\end{aligned}
$$

Moreover $f\left(j_{x}^{k} \psi\right)-\left(\int_{0}^{1} d t \psi E L(f)(t \psi) \psi\right) d x=d \beta\left(j_{x}^{2 k} \psi\right)$ where $\beta\left(j_{x}^{2 k} \psi\right) \in \Omega_{c}^{d-1}(M)$ is a compactly supported $d-1$ form.

\section{Explicit forms}

In this section, we derive the explicit expression of $\nabla F_{\varphi}$ and $F^{(\alpha)}(\varphi)$ in terms of $f$ when $M=\mathbb{R}^{d}$. Since the general expression is not very illuminating, let us start with the following simple example:

$$
F(\varphi)=\int_{M} h(x) \varphi^{4}(x)+g^{\mu \nu}(x) \partial_{\mu} \varphi(x) \partial_{\nu} \varphi(x) d x,
$$

where $h$ and $g^{\mu \nu}$ are smooth and compactly supported and $g^{\mu \nu}$ is symmetric. We compute

$$
\begin{aligned}
D F_{\varphi}(u) & =2 \int_{M} d x 2 h(x) \varphi^{3}(x) u(x)+g^{\mu v}(x) \partial_{\mu} \varphi(x) \partial_{\nu} u(x) \\
& =2 \int_{M} d x\left(2 h(x) \varphi^{3}(x)-\partial_{\nu}\left(g^{\mu \nu}(x) \partial_{\mu} \varphi(x)\right)\right) u(x),
\end{aligned}
$$

where we used integration by parts. Thus,

$$
\nabla F_{\varphi}(x)=4 h(x) \varphi^{3}(x)-2 \partial_{\nu}\left(g^{\mu v}(x) \partial_{\mu} \varphi(x)\right) .
$$

Moreover,

$$
\begin{aligned}
D^{2} F_{\varphi}(u, v)= & 2 \int_{M} d x u(x)\left(6 h(x) \varphi^{2}(x) v(x)\right. \\
& \left.-\partial_{\nu}\left(g^{\mu \nu}(x) \partial_{\mu} v(x)\right)\right)
\end{aligned}
$$

To write this as a distribution, we need to integrate over two variables:

$$
\begin{aligned}
D^{2} F_{\varphi}(u, v)= & 2 \int_{M^{2}} d x d y u(x) \delta(x-y)\left(6 h(y) \varphi^{2}(y) v(y)\right. \\
& \left.-\partial_{\nu}\left(g^{\mu \nu}(y) \partial_{\mu} v(y)\right)\right) .
\end{aligned}
$$

Now we can use integration by parts over $y$ to recover $v(y)$,

$$
D^{2} F_{\varphi}(u, v)=\sum_{\alpha} \int_{M^{2}} d x d y u(x) v(y) f^{\alpha}(\varphi)(y) \partial_{y}^{\alpha} \delta(x-y),
$$

where the non-zero $f^{\alpha}(\varphi)$ are 


$$
\begin{aligned}
f^{0}(\varphi)(y) & =12 \varphi^{2}(y), \\
f^{\mu}(\varphi)(y) & =-\partial_{\nu} g^{\mu \nu}(y), \\
f^{\mu \nu}(\varphi)(y) & =-g^{\mu v}(y) .
\end{aligned}
$$

More generally,

Proposition VI.19. If

$$
F(\varphi)=\int_{M} f\left(\varphi^{(\alpha)}(x)\right) d x
$$

then

$$
F^{\alpha}(\varphi)(x)=\sum_{\beta \leq \gamma}(-1)^{|\beta|}\left(\begin{array}{l}
\beta \\
\gamma
\end{array}\right) \partial_{y, \beta-\gamma} \frac{\partial^{2} f}{\partial \varphi^{(\alpha-\gamma)}(x) \partial \varphi^{(\beta)}(x)} .
$$

Proof. The proof is a straightforward generalization of the example. Indeed,

$$
\begin{aligned}
D F_{\varphi}(u) & =\sum_{\alpha} \int_{M} \frac{\partial f}{\partial \varphi^{(\alpha)}(x)} u^{(\alpha)}(x) d x \\
& =\sum_{\alpha}(-1)^{|\alpha|} \int_{M} u(x) \frac{\partial^{|\alpha|}}{\partial x^{\alpha}} \frac{\partial f}{\partial \varphi^{(\alpha)}(x)} d x,
\end{aligned}
$$

where we used an integration by parts to recover the Euler-Lagrange operator. The second derivative is

$$
D^{2} F_{\varphi}(u, v)=\sum_{\alpha \beta}(-1)^{|\alpha|} \int_{M} u(x) \frac{\partial^{|\alpha|}}{\partial x^{\alpha}}\left(\frac{\partial f^{2}}{\partial \varphi^{(\alpha)}(x) \partial \varphi^{(\beta)}(x)} v^{(\beta)}(x)\right) d x .
$$

We write this as a double integral

$$
D^{2} F_{\varphi}(u, v)=\sum_{\alpha \beta}(-1)^{|\alpha|} \int_{M^{2}} u(x) \delta(x-y) \partial_{y^{\alpha}}\left(\frac{\partial f^{2}}{\partial \varphi^{(\alpha)}(y) \partial \varphi^{(\beta)}(y)} v^{(\beta)}(y)\right) d x d y .
$$

A first integration by parts gives us

$$
D^{2} F_{\varphi}(u, v)=\sum_{\alpha \beta} \int_{M^{2}} u(x)\left(\frac{\partial f^{2}}{\partial \varphi^{(\alpha)}(y) \partial \varphi^{(\beta)}(y)} v^{(\beta)}(y)\right) \partial_{y^{\alpha}} \delta(x-y) d x d y .
$$

A second integration by parts isolates $v(y)$,

$$
D^{2} F_{\varphi}(u, v)=\sum_{\alpha \beta}(-1)^{|\beta|} \sum_{\gamma \leq \beta}\left(\begin{array}{l}
\beta \\
\gamma
\end{array}\right) \int_{M^{2}} d x d y u(x) v(y)\left(\partial_{y^{\beta-\gamma}} \frac{\partial f^{2}}{\partial \varphi^{(\alpha)}(y) \partial \varphi^{(\beta)}(y)}\right) \partial_{y^{\alpha+\gamma}} \delta(x-y) .
$$

If we calculate higher differentials $D^{k} F_{\varphi}\left(u_{1}, \ldots, u_{k}\right)$, we see that we always obtain products of smooth functions by derivatives of products of delta functions. This shows that the wavefront set of $F_{\varphi}^{(k)}$ is in the conormal $C_{k}$.

\section{PEETRE THEOREM FOR LOCAL AND MULTILOCAL FUNCTIONALS}

In this section, we propose an alternative characterization of local functionals in terms of a nonlinear Peetre theorem. We do not characterize the locality of the action $F$ but the locality of the Lagrangian density, which we denoted $\nabla F$ in Sec. IV D. We first state our theorems for local functionals and then we prove them for the case of multilocal functionals, which are a natural generalization of local functionals in quantum field theory. Our proof is inspired by recent studies on 
the Peetre theorem; ${ }^{108,109}$ however, it is formulated in the language of Bastiani smoothness and uses simpler assumptions than Slovák's paper. ${ }^{110}$

\section{A. Peetre theorem for local functionals}

Let $\Omega$ be some open set in a manifold $M$. We first begin with an alternative definition of a local map from $C^{\infty}(\Omega)$ to itself, which we call Peetre local.

Definition VII.1. A map $F: C^{\infty}(\Omega) \rightarrow C^{\infty}(\Omega)$ is Peetre local for every $x \in \Omega$; if $\varphi_{1}=\varphi_{2}$ on some neighborhood of $x$, then $F\left(\varphi_{1}\right)(x)=F\left(\varphi_{2}\right)(x)$.

The relation with the additivity condition is given by

Proposition VII.2. Let $F: C^{\infty}(\Omega) \rightarrow C^{\infty}(\Omega)$ be Peetre local. For every $\left(\varphi_{1}, \varphi_{2}\right) \in C^{\infty}(\Omega)^{2}$ if supp $\varphi_{1}$ and supp $\varphi_{2}$ do not meet, then for every $x \in \Omega$ and for all $\varphi$,

$$
F\left(\varphi_{1}+\varphi_{2}+\varphi\right)(x)=F\left(\varphi_{1}+\varphi\right)(x)+F\left(\varphi_{2}+\varphi\right)(x)-F(\varphi)(x) .
$$

Proof. If $x \notin\left(\operatorname{supp} \varphi_{1} \cup \operatorname{supp} \varphi_{2}\right)$, then $\varphi_{1}=\varphi_{2}=0$ in some neighborhood of $x$ it follows that $F\left(\varphi_{1}+\varphi_{2}+\varphi\right)(x)=F(0+0+\varphi)(x)=F(\varphi)(x)$ and $F\left(\varphi_{1}+\varphi\right)(x)+F\left(\varphi_{2}+\varphi\right)(x)-F(\varphi)(x)=2 F(\varphi)(x)$ $-F(\varphi)(x)=F(\varphi)(x)$ and hence Eq. (41) holds true.

If $x \in \operatorname{supp} \varphi_{1}$, then necessarily there is some neighborhood $U$ of $x$ on which $\left.\varphi_{2}\right|_{U}=0$ and hence $\varphi_{1}+\varphi_{2}+\left.\varphi\right|_{U}=\varphi_{1}+\left.\varphi\right|_{U}$ and $F\left(\varphi_{1}+\varphi_{2}+\varphi\right)(x)=F\left(\varphi_{1}+\varphi\right)(x)$. Also $F\left(\varphi_{1}+\varphi\right)(x)+F\left(\varphi_{2}+\varphi\right)(x)$ $-F(\varphi)(x)=F\left(\varphi_{1}+\varphi\right)(x)+F(\varphi)(x)-F(\varphi)(x)=F\left(\varphi_{1}+\varphi\right)(x)$, hence again Eq. (41) holds true. The case where $x \in \operatorname{supp} \varphi_{2}$ can be treated by similar methods which yields the final result.

The Peetre theorem for local functionals is

Theorem VII.3. Let $F: C^{\infty}(\Omega) \rightarrow C^{\infty}(\Omega)$ be a Bastiani smooth Peetre local map. Then, for every $\varphi \in C^{\infty}(\Omega)$, there is a neighborhood $V$ of $\varphi$ in $C^{\infty}(\Omega)$ and an integer $k$ such that for all $\psi$ such that $\varphi+\psi \in V, F(\varphi+\psi)(x)=c\left(j^{k} \psi_{x}\right)$ for some smooth function $c$ on $J^{k} \Omega$.

In other words, if $F$ is a Bastiani smooth Peetre local map, then, for every $g \in \mathcal{D}(M), \int_{M} F(\varphi) g$ is a Bastiani smooth local map in the sense of the rest of the paper. This relation between a priori different concepts of locality strongly supports the idea that our definition is a natural one.

If $F$ is only assumed to be a continuous local map, then a similar theorem exists for which the function $c$ is not necessarily smooth. These theorems are proved in the Sec. VII B for the more general case of multilocal functionals.

\section{B. Multilocal functionals and first Peetre theorem}

By generalizing Definition VII.1 of local maps, we can define multilocal maps. These maps appear naturally in quantum field theory as the product of several Lagrangian densities $\mathcal{L}\left(x_{1}\right) \ldots \mathcal{L}\left(x_{k}\right)$.

Definition VII.4. Let $k$ be an integer. A map $F: C^{\infty}(\Omega) \rightarrow C^{\infty}\left(\Omega^{k}\right)$ is $k$-local for every $\left(x_{1}, \ldots\right.$, $\left.x_{k}\right) \in \Omega^{k}$; if $\varphi_{1}=\varphi_{2}$ on some neighborhood of $\left\{x_{1}, \ldots, x_{k}\right\} \subset \Omega$, then $F\left(\varphi_{1}\right)\left(x_{1}, \ldots, x_{k}\right)=F\left(\varphi_{2}\right)\left(x_{1}\right.$, $\left.\ldots, x_{k}\right)$.

The multilocal maps are the maps that are $k$-local for some $k$. We emphasize that Peetre local maps in the sense of Definition VII.1 correspond with 1-local maps in the above sense. For $M$ a smooth manifold, we denote by $J^{p} M^{\otimes k}$ the bundle over $M^{k}$ whose fiber over a $k$-tuple of points $\left(x_{1}\right.$, $\left.\ldots, x_{k}\right) \in M^{k}$ is $J^{p} M_{x_{1}} \times \cdots \times J^{p} M_{x_{k}}$.

Theorem VII.5. Let $F: C^{\infty}(\Omega) \rightarrow C^{\infty}\left(\Omega^{k}\right)$ be a continuous $k$-local map. Then, for every $\varphi \in$ $C^{\infty}(\Omega)$, there is a neighborhood $V$ of $\varphi$ in $C^{\infty}(\Omega), p \in \mathbb{N}$, such that for all $\psi$ such that $\varphi+\psi \in V$,

$$
F(\varphi+\psi)\left(x_{1}, \ldots, x_{k}\right)=c\left(j^{p} \psi_{x_{1}}, \ldots, j^{p} \psi_{x_{k}}\right)
$$


for some function $c:\left.J^{p} M^{\llbracket k}\right|_{\left(M^{k} \backslash D_{k}\right)} \rightarrow M^{k}$, where $M^{k} \backslash D_{k}$ denotes the configuration space $M^{k}$ minus all diagonals.

Proof. Fix a $k$-tuple of points $\left(x_{1}, \ldots, x_{k}\right) \in \Omega^{k}$ and some compact neighborhood $K$ of $\left(x_{1}, \ldots\right.$, $x_{k}$ ) in $\Omega^{k}$. Continuity of $F$ implies that for all $\varepsilon>0$, there exists $\eta>0$ and a seminorm $\pi_{m, K^{\prime}}$ of $C^{\infty}(\Omega)$ such that $\pi_{m, K^{\prime}}\left(\varphi_{1}-\varphi_{2}\right) \leqslant \eta$ implies

$$
\sup _{\left(y_{1}, \ldots, y_{k}\right) \in K}\left|F\left(\varphi_{1}\right)\left(y_{1}, \ldots, y_{k}\right)-F\left(\varphi_{2}\right)\left(y_{1}, \ldots, y_{k}\right)\right| \leqslant \varepsilon .
$$

Assume that $\left(\varphi_{1}, \varphi_{2}\right)$ have the same $(m+1)$-jets at $\left\{x_{1}, \ldots, x_{k}\right\}$. Let $\left(\chi_{\lambda}\right)_{\lambda}$ be the family of compactly supported cutoff functions equal to 1 in some neighborhood of $X=\left\{x_{1}, \ldots, x_{k}\right\}$ defined in Lemma VII.6. It follows that $\varphi_{1, \lambda}=\varphi_{1} \chi_{\lambda}$ (respectively, $\varphi_{2, \lambda}=\varphi_{2} \chi_{\lambda}$ ) coincides with $\varphi_{1}$ (respectively, $\left.\varphi_{2}\right)$ near $\left\{x_{1}, \ldots, x_{k}\right\}$. Hence, for all $\lambda>0, F\left(\varphi_{1, \lambda}\right)\left(x_{1}, \ldots, x_{k}\right)=F\left(\varphi_{1}\right)\left(x_{1}, \ldots, x_{k}\right)$ and $F\left(\varphi_{2, \lambda}\right)\left(x_{1}\right.$, $\left.\ldots, x_{k}\right)=F\left(\varphi_{2}\right)\left(x_{1}, \ldots, x_{k}\right)$. Set $\varepsilon_{n}=\frac{1}{2^{n}}$, then there exists $\eta_{n}$ such that $\pi_{m, K^{\prime}}\left(\psi_{1}-\psi_{2}\right) \leqslant \eta_{n}$ implies

$$
\sup _{\left(y_{1}, \ldots, y_{k}\right) \in K}\left|F\left(\psi_{1}\right)\left(y_{1}, \ldots, y_{k}\right)-F\left(\psi_{2}\right)\left(y_{1}, \ldots, y_{k}\right)\right| \leqslant \frac{1}{2^{n}} .
$$

Therefore it suffices to find some sequence $\lambda_{n} \rightarrow 0$ such that $\pi_{m, K^{\prime}}\left(\varphi_{1, \lambda_{n}}-\varphi_{2, \lambda_{n}}\right) \leqslant \eta_{n}$. Since $\varphi_{1}-\varphi_{2}$ vanishes at order $m+1$ on the set $X=\left\{x_{1}, \ldots, x_{k}\right\}$, Lemma VII.6 yields the estimate

$$
\left|\pi_{m, K^{\prime}}\left(\varphi_{1, \lambda}-\varphi_{2, \lambda}\right)\right| \leqslant \tilde{C} \lambda \pi_{m+1, K}\left(\varphi_{1}-\varphi_{2}\right),
$$

which implies that

$$
\lim _{\lambda \rightarrow 0} \pi_{m, K^{\prime}}\left(\varphi_{1, \lambda}-\varphi_{2, \lambda}\right)=\lim _{\lambda \rightarrow 0} \pi_{m, K^{\prime}}\left(\left(\varphi_{1}-\varphi_{2}\right) \chi_{\lambda}\right)=0 .
$$

Finally, we obtain that if $\varphi_{1}, \varphi_{2}$ have the same $(m+1)$-jet at $X=\left\{x_{1}, \ldots, x_{k}\right\}$, then for all $n>0$,

$$
\left|F\left(\varphi_{1}\right)\left(x_{1}, \ldots, x_{k}\right)-F\left(\varphi_{2}\right)\left(x_{1}, \ldots, x_{k}\right)\right|=\left|F\left(\varphi_{1, \lambda_{n}}\right)\left(x_{1}, \ldots, x_{k}\right)-F\left(\varphi_{2, \lambda_{n}}\right)\left(x_{1}, \ldots, x_{k}\right)\right| \leqslant \frac{1}{2^{n}},
$$

which implies $F\left(\varphi_{1}\right)\left(x_{1}, \ldots, x_{k}\right)=F\left(\varphi_{2}\right)\left(x_{1}, \ldots, x_{k}\right)$.

Lemma VII.6. Let $X$ be any closed subset of $\mathbb{R}^{d}$. Let $\mathcal{I}^{m+1}\left(X, \mathbb{R}^{d}\right)$ denote the closed ideal of functions of regularity $C^{m+1}$ which vanish at order $m+1$ on $X$. Then there is a function $\chi_{\lambda} \in C^{\infty}\left(\mathbb{R}^{d}\right)$ parametrized by $\lambda \in(0,1]$ such that $\chi_{\lambda}=1$ (respectively, $\chi_{\lambda}=0$ ) when $d(x, X) \leqslant \frac{\lambda}{8}$ [respectively, $d(x, X) \geqslant \lambda]$ such that for all compact subset $K \subset \mathbb{R}^{d}$, there is a constant $\tilde{C}$ such that for every $\lambda \in$ $(0,1]$ and every $\varphi \in \mathcal{I}^{m+1}\left(X, \mathbb{R}^{d}\right)$,

$$
\pi_{m, K}(\chi \lambda \varphi) \leqslant \tilde{C} \lambda \pi_{m+1, K \cap\{d(x, X) \leqslant \lambda\}}(\varphi) .
$$

Proof. Choose $\phi \geqslant 0$ such that $\int_{\mathbb{R}^{d}} \phi(x) d^{d} x=1$ and $\phi=0$ if $|x| \geqslant \frac{3}{8}$. Set $\phi_{\lambda}=\lambda^{-d} \phi\left(\lambda^{-1}\right.$.), and set $\alpha_{\lambda}$ to be the characteristic function of the set $\left\{x\right.$ s.t. $\left.d(x, X) \leqslant \frac{\lambda}{2}\right\}$, then the convolution product $\chi_{\lambda}=$ $\phi_{\lambda} * \alpha_{\lambda}$ satisfies $\chi_{\lambda}(x)=1$ if $d(x, X) \leqslant \frac{\lambda}{8}$ and $\chi_{\lambda}(x)=0$ if $d(x, X) \geqslant \lambda$. Since by the Leibniz rule one has

$$
\partial^{\alpha}\left(\chi_{\lambda} \varphi\right)(x)=\sum_{|k| \leqslant|\alpha|}\left(\begin{array}{l}
\alpha \\
k
\end{array}\right) \partial^{k} \chi_{\lambda} \partial^{\alpha-k} \varphi(x),
$$

it suffices to estimate each term $\partial^{k} \chi_{\lambda} \partial^{\alpha-k} \varphi(x)$ of the above sum. For every multi-index $k$, there is some constant $C_{k}$ such that $\forall x \in \mathbb{R}^{d} \backslash X,\left|\partial_{x}^{k} \chi_{\lambda}\right| \leqslant \frac{C_{k}}{\lambda^{|k|}}$ and supp $\partial_{x}^{k} \chi_{\lambda} \subset\{d(x, X) \leqslant \lambda\}$. Therefore for all $\varphi \in \mathcal{I}^{m+1}\left(X, \mathbb{R}^{d}\right)$, for all $x \in \operatorname{supp} \partial_{x}^{k} \chi_{\lambda} \partial^{\alpha-k} \varphi$, and for $y \in X$ such that $d(x, X)=|x-y|$, we find that $\partial^{\alpha-k} \varphi$ vanishes at $y$ at order $|k|+1$. Indeed $\varphi$ vanishes at order $m+1$, hence $\partial^{\alpha-k} \varphi$ vanishes at order $m+1-|\alpha|+k \geqslant k+1$ since $|\alpha| \leqslant m$. Therefore,

$$
\partial_{x}^{\alpha-k} \varphi(x)=\sum_{|\beta|=|k|+1}(x-y)^{\beta} R_{\beta}(x),
$$


where the right-hand side is just the integral remainder in Taylor's expansion of $\partial^{\alpha-k} \varphi$ around $y$. Hence,

$$
\left|\partial^{k} \chi_{\lambda} \partial^{\alpha-k} \varphi(x)\right| \leqslant \frac{C_{k}}{\lambda^{|k|}} \sum_{|\beta|=|k|+1}\left|(x-y)^{\beta} R_{\beta}(x)\right| .
$$

It is easy to see that $R_{\beta}$ only depends on the jets of $\varphi$ of order $\leqslant m+1$. Hence

$$
\left|\partial^{k} \chi_{\lambda} \partial^{\alpha-k} \varphi(x)\right| \leqslant C_{k} \lambda \sup _{x \in K, d(x, X) \leqslant \lambda} \sum_{|\beta|=|k|+1}\left|R_{\beta}(x)\right|
$$

and the conclusion follows easily.

\section{The second Peetre theorem}

Theorem VII.7. Let $F: C^{\infty}(\Omega) \rightarrow C^{\infty}\left(\Omega^{k}\right)$ be a Bastiani smooth $k$-local map. Then, for every $\varphi \in C^{\infty}(\Omega)$, there is a neighborhood $V$ of $\varphi$ in $C^{\infty}(\Omega), p \in \mathbb{N}$, such that for all $\psi$ such that $\varphi+\psi \in V$,

$$
F(\varphi+\psi)\left(x_{1}, \ldots, x_{k}\right)=c\left(j^{p} \psi_{x_{1}}, \ldots, j^{p} \psi_{x_{k}}\right)
$$

for some smooth function c on $\left.J^{p} M^{\llbracket k}\right|_{\left(M^{k} \backslash D_{k}\right)}$ where $M^{k} \backslash D_{k}$ denotes the configuration space $M^{k}$ minus all diagonals.

Proof. Without loss of generality, we may assume that $M=\mathbb{R}^{d}$, and to go back to arbitrary manifolds, we use partitions of unity as in the proof of Lemma VI.6. The coordinates on the jet space $J^{p}\left(\mathbb{R}^{d}\right)$ are denoted by $\left(x, p^{\alpha}\right)_{|\alpha| \leqslant p}$. Let $\left(U_{1}, \ldots, U_{k}\right)$ be two by two disjoint open subsets of $\mathbb{R}^{d}$, then $U_{1} \times \cdots \times U_{k}$ is an open subset of $\left(\mathbb{R}^{d}\right)^{k} \backslash D_{k}$. We define the smooth map $\Phi:\left(x_{1}, \ldots, x_{k} ; p_{1}, \ldots, p_{k}\right) \in$ $\left.J^{p}\left(\mathbb{R}^{d}\right)^{\otimes k}\right|_{U_{1} \times \cdots \times U_{k}} \mapsto\left(\sum_{1 \leqslant i \leqslant k} \frac{p_{i, \alpha}}{\alpha !}\left(.-x_{i}\right)^{\alpha} \chi_{i}\left(.-x_{i}\right)\right) \in C^{\infty}\left(\mathbb{R}^{d}\right)$, where the functions $\chi_{i} \in C_{c}^{\infty}\left(\mathbb{R}^{d}\right)$ are cutoff functions equal to 1 near 0 and such that for all $\left(x_{1}, \ldots, x_{k}\right) \in U_{1} \times \cdots \times U_{k}$, the support of the functions $\chi_{i}\left(.-x_{i}\right)$ are disjoint on $\mathbb{R}^{d}$. Then the map sending $\left(x_{1}, \ldots, x_{k} ; p_{1}, \ldots, p_{k}\right),\left(y_{1}, \ldots\right.$, $\left.y_{k}\right)$ to $F\left(\varphi+\Phi\left(x_{1}, \ldots, x_{k} ; p_{1}, \ldots, p_{k}\right)\right)\left(y_{1}, \ldots, y_{k}\right)$ is smooth by smoothness of $F$ and $\Phi$. Hence, its pull-back on the diagonal $x_{1}=y_{1}, \ldots, x_{k}=y_{k}$ is also smooth and reads

$$
F\left(\varphi+\Phi\left(x_{1}, \ldots, x_{k} ; p_{1}, \ldots, p_{k}\right)\right)\left(x_{1}, \ldots, x_{k}\right)=c\left(x_{1}, \ldots, x_{k} ; p_{1}, \ldots, p_{k}\right)
$$

as the composition of smooth functions and it follows that $c$ is smooth on $\left.J^{p} M^{\otimes k}\right|_{U_{1} \times \cdots \times U_{k}}$.

\section{MULTI-VECTOR FIELDS AND GRADED FUNCTIONALS}

In the quantum theory of gauge fields, especially in the Batalin-Vilkovisky approach, it is necessary to deal not only with functionals as discussed above but also with multi-vector fields on the configuration space $E$ (assumed to be the space of sections of some vector bundle $B$ ). ${ }^{16}$ Such multivector fields can be seen as functionals on the graded space $T^{*}[1] E \doteq E \oplus E^{*}[1]$, where $E^{*} \doteq \Gamma\left(M, B^{*}\right)$ is the space of smooth sections. To make this notion precise, we use the ideas presented in Ref. 111 and characterize the "odd" space $E^{*}[1]$ through the space of functions on it, understood as multilinear smooth, totally antisymmetric, functionals. Then we shall make a conjectural claim on the meaning of locality in that context.

\section{A. Locality of functionals on graded space}

We consider a graded space $E_{0} \oplus E_{1}[1]$, where $E_{0}=\Gamma\left(M, B_{0}\right)$ and $E_{1}=\Gamma\left(M, B_{1}\right)$ are spaces of smooth sections of finite rank vector bundles $B_{0}$ and $B_{1}$ over $M$, respectively. Before giving formal definitions, let us explain the idea of our construction. We will first define the space $\mathcal{O}\left(E_{0} \oplus E_{1}[1]\right)$ to be space of maps from $E_{0}$ to $\mathcal{A}$, where

$$
\mathcal{A} \doteq \prod_{k=0}^{\infty} \mathcal{A}^{k} \doteq \prod_{k=0}^{\infty} \Gamma_{a}^{\prime}\left(M^{k}, B_{1}^{\otimes k}\right),
$$


satisfying an appropriate smoothness condition. Let us clarify the notation $\Gamma_{a}^{\prime}$. We first define the iterated wedge product of $k$ elements $u_{1}, \ldots, u_{k}$ of the space of distributional sections $\Gamma^{\prime}\left(M, B_{1}\right)$ by

$$
\left\langle u_{1} \wedge \cdots \wedge u_{k}, h_{1} \otimes \cdots \otimes h_{k}\right\rangle=\sum_{\sigma}(-1)^{\sigma}\left\langle u_{1}, h_{\sigma(1)}\right\rangle \ldots\left\langle u_{k}, h_{\sigma(k)}\right\rangle,
$$

where $h_{1}, \ldots, h_{k}$ are sections in $\Gamma\left(M, B_{1}\right)$ and $\sigma$ runs over the permutations of $\{1, \ldots, k\}$. Then, the $k$ th exterior power $\Lambda^{k} \Gamma^{\prime}\left(M, B_{1}\right)$ is the vector space of finite sums of such iterated wedge products and $\Gamma_{a}^{\prime}\left(M^{k}, B_{1}^{\bowtie k}\right)$ is the completion of $\Lambda^{k} \Gamma^{\prime}\left(M, B_{1}\right)$ with respect to the topology of $\Gamma^{\prime}\left(M, B_{1}\right)^{\hat{\otimes}_{\pi} k} \cong$ $\Gamma^{\prime}\left(M^{k}, B_{1}^{\otimes k}\right)$ where all the duals are strong. The subscript " $a$ " stands for antisymmetry.

In the case of multilinear symmetric functions, we can identify a $k$-linear map $f\left(h_{1}, \ldots, h_{k}\right)$ of $k$ variables with a polynomial map of one variable $f(h, \ldots, h)$ by using the polarization identity. There is no polarization identity in the antisymmetric case and we must consider a function $F: E_{0} \rightarrow \mathcal{A}^{k}$ as a function of one variable $\varphi_{0}$ in $E_{0}$ and $k$ variables $\left(h_{1}, \ldots, h_{k}\right)$ in $E_{1}$ (or a variable in $H \in E_{1}^{\hat{\otimes}_{\pi} k}$ ). Then, we can identify a function $F: E_{0} \rightarrow \mathcal{A}^{k}$ and the function $\tilde{F}: E_{0} \times E_{1}^{\hat{\otimes}_{\pi} k} \rightarrow \mathbb{K}$ defined by

$$
\tilde{F}\left(\varphi_{0} ; h_{1} \otimes \cdots \otimes h_{k}\right)=F\left(\varphi_{0}\right)\left(h_{1} \otimes \cdots \otimes h_{k}\right) .
$$

This motivates the following:

Definition VIII.1. Let $M$ be a smooth manifold, $\left(B_{0}, B_{1}\right)$ are smooth vector bundles on $M$, and $E_{0}=\Gamma\left(M, B_{0}\right), E_{1}=\Gamma\left(M, B_{1}\right)$ are spaces of smooth sections of the respective bundles. We say that a function $F$ from $E_{0}$ to $\mathcal{A}^{k}$ is an element of $\mathcal{O}^{k}\left(E_{0} \oplus E_{1}[1]\right)$ if there exists a Bastiani smooth map $\tilde{F}: E_{0} \times E_{1}^{\hat{\otimes}_{\pi} k} \rightarrow \mathbb{K}$ which is linear in $E_{1}^{\hat{\otimes}_{\pi} k}$ and antisymmetric with respect to the natural action of permutations on $E_{1}^{\hat{\otimes}_{\pi} k}$ such that

$$
\tilde{F}\left(\varphi_{0} ; h_{1} \otimes \cdots \otimes h_{k}\right)=F\left(\varphi_{0}\right)\left(h_{1} \otimes \cdots \otimes h_{k}\right) .
$$

We denote by $\mathcal{O}\left(E_{0} \oplus E_{1}[1]\right)$ the direct product of all $\mathcal{O}^{k}\left(E_{0} \oplus E_{1}[1]\right)$, over $k \in \mathbb{N}_{0}$ and set $\mathcal{O}^{0}\left(E_{0} \oplus\right.$ $\left.E_{1}[1]\right) \equiv \mathbb{K}$.

Let us now discuss the notion of derivative for the type of functionals introduced above. Clearly, if $F$ belongs to $\mathcal{O}\left(E_{0} \oplus E_{1}[1]\right)$, there are two natural ways to differentiate it. In the first instance, we can differentiate $\tilde{F}$ in the sense of Bastiani in the first variable $\left(\varphi \in E_{0}\right)$ and we denote this derivative as

$$
D_{0} F_{(\varphi ; u)}(g) \doteq D \tilde{F}_{(\varphi, u)}(g, 0),
$$

where $u \in E_{1}^{\hat{\otimes}_{\pi} k} \rightarrow \mathbb{K}, g \in E_{0}$, or $\frac{\delta}{\delta \varphi_{0}} F$.

\section{B. The contraction operation}

Let us now consider contraction of the graded part with some $h \in E_{1}$, sometimes referred to as derivations with respect to odd variables. This concept is needed in order to define the Koszul complex and the Chevalley-Eilenberg complex in the Batalin-Vilkovisky formalism in infinite dimension. The definition is spelled out below.

Definition VIII.2. Let $F \in \mathcal{O}^{k}\left(E_{0} \oplus E_{1}[1]\right), h \in E_{1}$. The contraction of $F$ by $h$ is defined, for every integer $k>0$ and $u \in E_{1}^{\otimes k-1}$, by

$$
\begin{aligned}
& \left\langle\iota_{h} F, u\right\rangle=\tilde{F}(h \otimes u) \\
& \text { and } \iota_{h} F=0 \quad \text { if } F \in \mathcal{A}^{0} .
\end{aligned}
$$

In particular, $\iota_{h} F=\langle\tilde{F}, h\rangle$ if $F \in \mathcal{A}^{1}$. We extend this definition to $\mathcal{A}$ by linearity.

In view of (43) and the definition of $\mathcal{O}^{k}\left(E_{0} \oplus E_{1}[1]\right)$, it is clear that $\iota_{h} F \in \mathcal{O}^{k-1}\left(E_{0} \oplus E_{1}[1]\right)$ for all $F \in \mathcal{O}^{k}\left(E_{0} \oplus E_{1}[1]\right)$. Equation (43) allows us also to make sense of a second important operation on $\mathcal{O}\left(E_{0} \oplus E_{1}[1]\right)$. 
Definition VIII.3. The wedge product $\wedge: \mathcal{O}^{k}\left(E_{0} \oplus E_{1}[1]\right) \times \mathcal{O}^{k^{\prime}}\left(E_{0} \oplus E_{1}[1]\right) \rightarrow \mathcal{O}^{k+k^{\prime}}\left(E_{0} \oplus E_{1}[1]\right)$ is defined by

$$
(\widetilde{F \wedge G})\left(u_{1}, \ldots, u_{k+k^{\prime}}\right)=\sum_{\sigma} \operatorname{sgn}(\sigma) \tilde{F}\left(u_{\sigma(1)}, \ldots, u_{\sigma(k)}\right) \tilde{G}\left(u_{\sigma(k+1)}, \ldots, u_{\sigma\left(k+k^{\prime}\right)}\right.
$$

(where the sum runs over $k-k^{\prime}$ shuffles) and extended by linearity on $\mathcal{O}\left(E_{0} \oplus E_{1}[1]\right) \times \mathcal{O}\left(E_{0} \oplus E_{1}[1]\right)$.

Again, in view of (43) and the definition of $\mathcal{O}\left(E_{0} \oplus E_{1}[1]\right)$, it is clear that the wedge product of an element in $\mathcal{O}^{k}\left(E_{0} \oplus E_{1}[1]\right)$ with an element in $\mathcal{O}^{k^{\prime}}\left(E_{0} \oplus E_{1}[1]\right)$ is an element in $\mathcal{O}^{k+k^{\prime}}\left(E_{0} \oplus E_{1}[1]\right)$. The contraction and wedge product satisfy the following relation on $\mathcal{O}\left(E_{0} \oplus E_{1}[1]\right)$ :

Lemma VIII.4. The contraction satisfies the graded Leibniz rule if $F \in \mathcal{O}^{k}\left(E_{0} \oplus E_{1}[1]\right), G \in$ $\mathcal{O}\left(E_{0} \oplus E_{1}[1]\right)$ and $h \in E_{1}$, then

$$
\iota_{h}(F \wedge G)=\left(\iota_{h} F\right) \wedge G+(-1)^{k} F \wedge \iota_{h} G .
$$

Let us now discuss the notion of support which is the appropriate generalization of the notion of support for graded functionals, generalizing the definitions in Sec. III A.

Definition VIII.5. Let $F \in \mathcal{O}^{k}\left(U \oplus E_{1}[1]\right)$ be a graded functional, with $U$ being an open subset of $E_{0}$. The support of $F$ is defined by $\operatorname{supp} F=\overline{A \cup B}$, where

$$
\begin{aligned}
& A=\bigcup_{\left(h_{1}, \ldots, h_{k}\right) \in E_{1}^{k}} \operatorname{supp}\left(\varphi \mapsto\left(\iota_{h_{1}} \ldots \iota_{h_{k}} F\right)(\varphi)\right), \\
& B=\bigcup_{\varphi \in U,\left(h_{1}, \ldots, h_{k-1}\right) \in E_{1}^{k-1}} \operatorname{supp}\left(h \mapsto\left(\iota_{h_{1}} \ldots \iota_{h_{k-1}} F(\varphi, h)\right)\right) .
\end{aligned}
$$

\section{Some conjectures on local graded functionals}

Let $F \in \mathcal{O}^{k}\left(E_{0} \oplus E_{1}[1]\right)$ be such that the WF set of both $\left(\iota_{h_{1}} \ldots \iota_{h_{k}} F\right)_{\varphi}^{(1)}$ and $\iota_{h_{1}} \ldots \iota_{h_{k-1}} F(\varphi,$. is empty for all $\varphi \in U$ and $\left(h_{1}, \ldots, h_{k}\right) \in E_{1}^{k}$. We conjecture that some version of Lemmas VI.2 and VI.9 should hold in the graded case. The "standard" characterization of locality for a functional $F \in \mathcal{O}^{k}\left(E_{0} \oplus E_{1}[1]\right)$ is the requirement that $F$ is compactly supported, and for each $\left(\varphi ; u_{1}, \ldots, u_{k}\right) \in$ $E_{0} \times E_{1}^{k}$, there exists $i_{0}, \ldots, i_{k} \in \mathbb{N}$ such that

$$
F\left(\varphi ; u_{1}, \ldots, u_{k}\right)=\int_{M} \alpha\left(j_{x}^{i_{0}}(\varphi), j_{x}^{i_{1}}\left(u_{1}\right), \ldots, j_{x}^{i_{k}}\left(u_{k}\right)\right),
$$

where $\alpha$ is a density-valued function on the jet bundle. To conclude, we conjecture some graded analog of Theorem VI.3 whose formulation would be as follows: Let $U$ be an open subset of $E_{0}$ and $F \in \mathcal{O}^{k}\left(U \oplus E_{1}[1]\right)$ be a graded functional. Assume that we have the following:

1. $F$ is additive in some suitable sense, still to be written with care (conceivably this would be additivity of $\tilde{F}$ as a function of several variables).

2. $\left(\iota_{h_{1}} \ldots \iota_{h_{k}} F\right)_{\varphi}^{(1)}$ and $\iota_{h_{1}} \ldots \iota_{h_{k-1}} F(\varphi,$.$) have an empty wave front set for all \varphi \in U$ and $\left(h_{1}, \ldots, h_{k}\right) \in E_{1}^{k}$, and the maps $(\varphi, u) \mapsto\left(\iota_{h_{1}} \ldots \iota_{h_{k}} F\right)_{\varphi}^{(1)}, \iota_{h_{1}} \ldots \iota_{h_{k-1}} F(\varphi,$.$) are Bastiani smooth$ from $U \times \bigoplus_{k \in \mathbb{N}} E_{1}^{\hat{\otimes}_{\pi} k}$ to $\Gamma_{c}\left(M, B_{0}^{*}\right)$ and $\Gamma_{c}\left(M, B_{1}^{*}\right)$, respectively. Here $B_{0}^{*}$ and $B_{1}^{*}$ denote dual bundles.

Then, for every $\varphi \in U, u \in \bigoplus_{k \in \mathbb{N}} E_{1}^{\hat{\otimes}_{\pi} k}$, there is a neighborhood $V$ of the origin in $E_{0}$, an integer $N$, and a smooth $\mathbb{K}$-valued function $f$ on the $N$-jet bundle such that

$$
F\left(\varphi+\psi ; v_{1} \otimes \cdots \otimes v_{k}\right)=\int_{M} \alpha\left(j_{x}^{i_{0}}(\psi), j_{x}^{i_{1}}\left(v_{1}\right), \ldots, j_{x}^{i_{k}}\left(v_{k}\right)\right),
$$

for every $\psi \in V$ and some $i_{0}, \ldots, i_{k}<N$. 


\section{ACKNOWLEDGMENTS}

We are very grateful to the Institut Poincare for making this work possible through the grant and facilities it offered us within the framework of its "Research in Paris" program. We thank the CNRS Groupement de Recherche Renormalisation for its financial support. N.V.D. acknowledges No. ANR16-CE40-0012-01 grant for financial support. We are extremely grateful to Yoann Dabrowski for his very generous help during all the phases of this work. We thank Romeo Brunetti and Pedro Ribeiro for very useful discussions.

${ }^{1}$ J. Schwinger, Proc. Natl. Acad. Sci. U. S. A. 37, 452 (1951).

${ }^{2}$ J. Schwinger, Proc. Natl. Acad. Sci. U. S. A. 37, 455 (1951).

${ }^{3}$ W. Kohn and L. J. Sham, Phys. Rev. 140, A1133 (1965).

${ }^{4}$ E. Engel and R. M. Dreizler, Density Functional Theory: An Advanced Course (Springer, Heidelberg, 2011).

${ }^{5}$ K. Rejzner, Perturbative Algebraic Quantum Field Theory: An Introduction for Mathematicians (Springer, Cham, 2016).

${ }^{6} \mathrm{M}$. Dütsch and K. Fredenhagen, in Rigorous Quantum Field Theory: A Festschrift for Jacques Bros, Volume 251 of Progress in Mathematics, edited by A. B. de Monvel, D. Buchholz, D. Iagolnitzer, and U. Moschella (Birkhäuser, Basel, 2006), pp. 113-124.

${ }^{7}$ R. Brunetti, K. Fredenhagen, and K. Rejzner, Commun. Math. Phys. 345, 741 (2016).

${ }^{8}$ D. Bahns and K. Rejzner, "The quantum sine-Gordon model of perturbative AQFT," Commun. Math. Phys. (published online).

${ }^{9}$ L. Lempert and N. Zhang, Acta Math. 193, 241 (2004).

${ }^{10}$ L. Dickey, Soliton Equations and Hamiltonian Systems (World Scientific, 2003), Vol. 26.

${ }^{11}$ J. Lee and R. M. Wald, J. Math. Phys. 31, 725 (1990).

${ }^{12}$ R. M. Wald, General Relativity (The University of Chicago Press, Chicago, 1984).

${ }^{13}$ J. D. Stasheff, Contemp. Math. 219, 195 (1998).

${ }^{14}$ G. Barnich, F. Brandt, and M. Henneaux, Phys. Rep. 338, 439 (2000).

${ }^{15}$ I. Khavkine, Classical Quantum Gravity 32, 185019 (2015).

${ }^{16}$ K. Fredenhagen and K. Rejzner, Commun. Math. Phys. 314, 93 (2012).

${ }^{17}$ R. Brunetti, M. Dütsch, and K. Fredenhagen, Adv. Theor. Math. Phys. 13, 1541 (2009).

${ }^{18}$ R. Brunetti and K. Fredenhagen, in Quantum Field Theory on Curved Spacetimes: Concepts and Mathematical Foundations, Volume 786 of Lecture Notes in Physics, edited by C. Bär and K. Fredenhagen (Springer, Berlin, 2009), pp. 129-155.

${ }^{19}$ In this article, all manifolds shall be assumed to be paracompact.

${ }^{20}$ R. Brunetti, K. Fredenhagen, and P. L. Ribeiro, e-print arXiv:1209.2148 (2012).

${ }^{21}$ Y. Dabrowski, e-print arXiv:1411.3012 (2014).

${ }^{22}$ Y. Dabrowski, e-print arXiv:1412.1749 (2014).

${ }^{23}$ H. Epstein and V. Glaser, Ann. Inst. Henri Poincaré, Ser. A 19, 211 (1973), available at http://www.numdam.org/ item?id=AIHPA_1973_19_3_211_0.

${ }^{24}$ A. S. Wightman, Fortschr. Phys. 44, 143 (1996).

${ }^{25}$ M. Martellini, Il Nuovo Cimento A 67, 305 (1982).

${ }^{26}$ M. Dütsch and K. Fredenhagen, Commun. Math. Phys. 219, 5 (2001).

${ }^{27}$ L. Schwartz, Théorie des Distributions, 2nd ed. (Hermann, Paris, 1966).

${ }^{28}$ A. Kriegl and P. W. Michor, The Convenient Setting of Global Analysis (American Mathematical Society, Providence, 1997).

${ }^{29}$ E. C. G. Stueckelberg, Phys. Rev. 81, 130 (1951).

${ }^{30}$ J. Horváth, Topological Vector Spaces and Distributions (Addison-Wesley, Reading, 1966).

${ }^{31}$ F. Trèves, Topological Vector Spaces, Distributions and Kernels (Dover, New York, 2007).

${ }^{32}$ L. Hörmander, The Analysis of Linear Partial Differential Operators I: Distribution Theory and Fourier Analysis, 2nd ed. (Springer Verlag, Berlin, 1990).

${ }^{33}$ Since an open set is absorbing (Ref. 30, p. 80), for every $x \in U$ and every $v \in E$ there is an $\epsilon>0$ such that $x+t v \in U$ if $|t|$ $<\epsilon$. Thus, $f(x+t v)$ is well defined for every $t$ such that $|t|<\epsilon$.

${ }^{34}$ A. Shapiro, J. Optim. Theory Appl. 66, 477 (1990).

${ }^{35}$ G. A. Ladas and V. Laskhmikantham, Differential Equations in Abstract Spaces (Academic Press, New York, 1972).

${ }^{36}$ A. J. Kurdila and M. Zabarankin, Convex Functional Analysis (Birkhäuser, Basel, 2005).

${ }^{37}$ H. Sagan, Introduction to the Calculus of Variations (Dover, New York, 1992).

${ }^{38}$ S. Yamamuro, Differential Calculus in Topological Linear Spaces, Volume 374 of Lecture Notes in Mathematics (Springer, Berlin, 1974).

${ }^{39}$ A. Bastiani, J. Anal. Math. 13, 1 (1964).

${ }^{40}$ R. S. Hamilton, Bull. Am. Math. Soc. 7, 65 (1982).

${ }^{41}$ A. Bastiani, "Differentiabilité dans les espaces localement convexes. Distructures," Ph.D. thesis, Université de Paris, 1962.

${ }^{42}$ K.-H. Neeb, in Infinite Dimensional Kähler Manifolds, edited by A. Huckleberry and T. Wurzbacher (Birkhäuser, Basel, 2001), pp. 131-178.

43 J.-P. Penot, Stud. Math. 47, 1 (1973).

${ }^{44}$ H. H. Keller, Differential Calculus in Locally Convex Spaces, Volume 417 of Lecture Notes in Mathematics (Springer, Berlin, 1974).

${ }^{45}$ V. I. Averbukh and O. G. Smolyanov, Russ. Math. Surv. 23, 67 (1968).

${ }^{46}$ W. Gähler, Grundstrukturen der Analysis (Birkhäuser, Basel, 1978), Vol. 2. 
${ }^{47}$ P. Ver Eecke, Fondements du Calcul Différentiel (Presses Universitaires de France, Paris, 1983).

${ }^{48}$ M. Z. Nashed, in Nonlinear Functional Analysis and Applications, edited by L. B. Rall (Academic Press, New York, 1971), pp. 103-359.

${ }^{49}$ M. C. Abbati and A. Manià, J. Geom. Phys. 29, 35 (1999).

${ }^{50}$ P. W. Michor, Manifolds of Differentiable Mappings (Shiva Publishing Ltd, Orpington, 1980).

${ }^{51}$ J. Milnor, in Relativity, Groups, and Topology II, Volume 40 of Les Houches, edited by B. S. DeWitt and R. Stora (North Holland, Amsterdam, 1983), pp. 1007-1057.

${ }^{52}$ B. Khesin and R. Wendt, The Geometry of Infinite-Dimensional Groups (Springer, Berlin, 2009).

${ }^{53}$ W. Bertram, Differential Geometry, Lie Groups and Symmetric Spaces Over General Base Fields and Rings, Memoirs of the American Mathematical Society (American Mathematical Society, Providence, 2008), Vol. 192.

${ }^{54}$ J. Szilasi and R. L. Lovas, in Handbook of Global Analysis, edited by D. Krupka and D. Saunders (Oxford University Press, Oxford, 2008), pp. 1069-1114

${ }^{55}$ R. Dahmen, Forum Math. 27, 2957 (2015).

${ }^{56}$ A. D. Michal, Proc. Natl. Acad. Sci. U. S. A. 24, 340 (1938).

${ }^{57}$ H. Massam and S. Zlobec, Math. Program. 7, 144 (1974).

${ }^{58}$ A. Ehresmann, Category Theory Yesterday, Today (and Tomorrow?): A Colloquium in Honour of Jean Benabou (ENS, Paris, 2011).

${ }^{59}$ J. L. Kelley, General Topology, Volume 27 of Graduate Texts in Mathematics, Revised 3rd ed. (Springer-Verlag, New York, 1955).

${ }^{60}$ N. Bourbaki, Elements of Mathematics: General Topology. Part 2 (Addison-Wesley, Reading, 1966)

${ }^{61}$ N. Bourbaki, Elements of Mathematics: Topological Vector Spaces (Springer, Berlin, 2003).

${ }^{62}$ M. Reed and B. Simon, Methods of Modern Mathematical Physics: I. Functional Analysis, 2nd ed. (Academic Press, New York, 1980).

${ }^{63}$ K.-H. Neeb, Ann. Inst. Fourier 61, 1839 (2011).

${ }^{64}$ N. Bourbaki, Eléments de Mathématique: Intégration, 2nd ed. (Hermann, Paris, 1965), Chaps. 1 and 4.

${ }^{65}$ N. Bourbaki, "Intégration vectorielle," in Eléments de Mathématique: Intégration (Hermann, Paris, 1959), Chap. 6.

${ }^{66}$ H. Glöckner, Orlicz Centenary Volume, Volume 55 of Banach Center Publications (Polish Academy of Sciences, Warsaw, 2002), pp. 43-59.

${ }^{67}$ K.-H. Neeb, Monastir Summer School: Infinite-Dimensional Lie Groups, Preprint 2433 (TU Darmstadt, Darmstadt, 2006).

${ }^{68}$ H. Glöckner, Infinite-Dimensional Lie Groups, Lecture Notes (TU Darmstadt, Darmstadt, 2005).

${ }^{69}$ E. Shult and D. Surowski, Algebra: A Teaching and Source Book (Springer, Berlin, 2015).

${ }^{70}$ N. Bourbaki, Elements of Mathematics: Algebra I (Springer, Berlin, 1989), Chaps 1-3.

${ }^{71}$ R. A. Ryan, Applications of Topological Tensor Products to Infinite Dimensional Topology (Trinity College, Dublin, 1980).

${ }^{72}$ G. Köthe, Topological Vector Spaces II (Springer Verlag, New York, 1979).

${ }^{73}$ A. Grothendieck, Produits Tensoriels Topologiques et Espaces Nucléaires, Volume 16 of Memoirs of the American Mathematical Society (American Mathematical Society, Providence, 1955).

${ }^{74}$ N. N. Tarkhanov, The Analysis of Solutions of Elliptic Equations (Kluwer Academic Publishers, Dordrecht, 1997).

${ }^{75}$ M. Grosser, Novi Sad J. Math. 38, 121 (2008), avilable at http://www.dmi.uns.ac.rs/nsjom/Papers/38_3/NSJOM_38_3_121 -128.pdf.

${ }^{76}$ H. Glöckner, J. Funct. Anal. 194, 347 (2002).

${ }^{77}$ C. Wockel, Central Extensions of Gauge Groups (Diplomarbeit, Technische Universität Darmstadt, 2003).

${ }^{78}$ C. Wockel, "Infinite-dimensional Lie theory for gauge groups," Ph.D. thesis, Technische Universität Darmstadt, 2006.

${ }^{79}$ C. Brouder, N. V. Dang, and F. Hélein, J. Phys. A: Math. Theor. 47, 443001 (2014).

${ }^{80}$ J.-F. Colombeau and R. Meise, Functional Analysis, Holomorphy, and Approximation Theory, Volume 843 of Lecture Notes in Mathematics (Springer, Berlin, 1981), pp. 195-216.

${ }^{81}$ K. Fredenhagen and K. Rejzner, Commun. Math. Phys. 317, 697 (2013).

${ }^{82}$ K. Fredenhagen and K. Rejzner, "Perturbative algebraic quantum field theory," in Mathematical Aspects of Quantum Field Theories, edited by D. Calaque and T. Strobl (Springer, 2015), pp. 17-55.

${ }^{83}$ C. Bär and R. T. Wafo, Math. Phys. Anal. Geom. 18, 7 (2015).

${ }^{84}$ T. Hirai, H. Shimomura, N. Tatsuuma, and E. Hirai, J. Math. Kyoto Univ. 41, 475 (2001).

${ }^{85}$ Y. Dabrowski and C. Brouder, Commun. Math. Phys. 332, 1345 (2014).

${ }^{86}$ S. Hollands and R. M. Wald, Commun. Math. Phys. 223, 289 (2001).

${ }^{87}$ K. Rejzner, "Batalin-Vilkovisky formalism in locally covariant field theory," Ph.D. thesis, University of Hamburg, 2011.

${ }^{88}$ M. M. Rao, in Measure Theory Oberwolfach 1979, Volume 794 of Lecture Notes in Mathematics (Springer, Berlin, 1980), pp. 484-496.

${ }^{89}$ A. G. Pinsker, C. R. (Dokl.) Acad. Sci. URSS 18, 399 (1938).

${ }^{90}$ I. M. Gel'fand and N. Y. Vilenkin, Generalized Functions: Applications of Harmonic Analysis (Academic Press, New York, 1964), Vol. IV

${ }^{91}$ M. Dütsch and K. Fredenhagen, Rev. Math. Phys. 16, 1291 (2004).

92 K. J. Keller, J. Math. Phys. 50, 103503 (2009).

${ }^{93}$ R. V. Chacon and N. Friedman, Arch. Ration. Mech. Anal. 18, 230 (1965).

${ }^{94}$ J. Batt, Stud. Math. 48, 146 (1973).

${ }^{95}$ S. H. Fesmire, in Functional Analysis and Its Applications, Volume 399 of Lecture Notes in Mathematics (Springer, Berlin, 1974), pp. 129-148.

${ }^{96}$ A. de Korvin and C. E. Roberts, Jr., Pac. J. Math. 92, 329 (1981).

${ }^{97}$ K. Lermer, Stud. Math. 129, 97 (1998).

${ }^{98}$ Z. Ercan, Czech. Math. J. 49, 187 (1999). 
${ }^{99}$ H. G. R. Millington, e-print arXiv:0706.4281 (2007).

100 J. A. Navarro, Math. Proc. Cambridge Philos. Soc. 117, 371 (1995).

${ }^{101}$ R. Moosa, preprint arXiv:math/0405563 (2004).

102 J. Kantor, Mém. Soc. Math. Fr. 53, 5 (1977).

${ }^{103}$ Since all manifolds in this article are assumed to be paracompact, for every property $T$ on the set of all open subsets on $M$, provided that (i) open subsets of subsets satisfying $T$ satisfy $T$ and (ii) every point of $M$ admits a neighborhood that satisfies property $T$, there exists an open cover $\left(U_{i}\right)_{i \in I}$ made of subsets satisfying the property $T$ that admits a partition of unity $\left(\chi_{i}\right)_{i \in I}$ relative to it.

${ }^{104}$ That is, $\mathrm{t}_{x}^{k} \circ \mathfrak{J}_{x}^{k}(f)=f$.

105 N. V. Dang, Ann. Henri Poincaré 17, 819 (2016).

${ }^{106}$ C. Brouder, N. V. Dang, and F. Hélein, Stud. Math. 232, 201 (2016); e-print arXiv:1409.7662.

107 J. M. Lee, Introduction to Smooth Manifolds (Springer, New York, 2003).

108 J. Navarro and J. B. Sancho, e-print arXiv:1411.7499 (2014).

109 J.-P. Brasselet and M. J. Pflaum, Ann. Math. 167, 1 (2008).

${ }^{110}$ J. Slovák, Ann. Global Anal. Geom. 6, 273 (1988).

${ }^{111}$ K. Rejzner, Rev. Math. Phys. 23, 1009 (2011). 